\title{
Next-to-next-to-leading order prediction for the photon-to-pion transition form factor
}

\author{
B. Melić* \\ Institut für Physik, Universität Mainz, D-55099 Mainz, Germany \\ and Institut für Theoretische Physik und Astrophysik, Universität Würzburg, D-97074 Würzburg, Germany
}

D. Müller and K. Passek-Kumerički*

Fachbereich Physik, Universität Wuppertal, D-42097 Wuppertal, Germany

(Received 4 February 2003; published 22 July 2003)

\begin{abstract}
We evaluate the next-to-next-to-leading order corrections to the hard-scattering amplitude of the photon-topion transition form factor. Our approach is based on the predictive power of the conformal operator product expansion, which is valid for a vanishing $\beta$ function in the so-called conformal scheme. The Wilson coefficients appearing in the nonforward kinematics are then entirely determined from those of the polarized deep inelastic scattering known to next-to-next-to-leading order accuracy. We propose different schemes to include explicitly also the conformal symmetry breaking term proportional to the $\beta$ function and discuss numerical predictions calculated in different kinematical regions. It is demonstrated that the photon-to-pion transition form factor can provide a fundamental testing ground for our QCD understanding of exclusive reactions.
\end{abstract}

DOI: 10.1103/PhysRevD.68.014013

PACS number(s): 12.38.Bx, 11.10.Hi, 13.40.Gp, 13.60.Le

\section{INTRODUCTION}

At a sufficiently large scale, exclusive QCD reactions factorize into a perturbative calculable partonic hard-scattering amplitude and universal hadron distribution amplitudes [1-3]. The study of such reactions offers the possibility of directly exploring nonperturbative features of hadrons at the amplitude level, as well as of testing our understanding of the amplitude factorization. Unfortunately, exclusive reactions are still challenging to both experimentalists and theoreticians, and the onset of the perturbative approach is a controversial topic in the literature.

The photon-to-pion transition form factor, appearing in the two-photon amplitude of the process $\gamma^{*}\left(q_{1}\right) \gamma^{(*)}\left(q_{2}\right)$ $\rightarrow \pi^{0}(P)$, can serve for a thorough study of the mentioned problem. In this process the partonic content of a meson is probed only by the electromagnetic interaction. Furthermore, since we require that the meson is produced at lightlike distances, i.e., that at least one photon is far off shell, this process belongs to quite a large class of two-photon processes calculable by means of the operator product expansion (OPE) [4]. Deeply virtual Compton scattering (DVCS), deeply inelastic lepton-hadron scattering (DIS), and the production of various hadronic final states by photon-photon fusion belong to this class of processes. Such processes can be described by a general scattering amplitude given by the time-ordered product of two electromagnetic currents sandwiched between the hadronic states. For a specific process, the generalized Bjorken kinematics at the light cone can be reduced to the corresponding kinematics, while the particular hadron content of the process is reflected in the nonperturbative part of the amplitude. Hence, the generalized hardscattering amplitude enables us to relate predictions of different two-photon processes on the partonic level.

\footnotetext{
*On leave of absence from the Rudjer Bošković Institute, Zagreb, Croatia.
}

In the leading twist- 2 approximation, the light-cone OPE approach is equivalent to the collinear factorization scheme [1-3]. The transition form factor factorizes as a convolution of the hard-scattering amplitude $T$ and the pion distribution amplitude $\phi$, with respect to the momentum fraction $x$ :

$$
F_{\gamma \pi}(\omega, Q)=f_{\pi} T\left(\omega, x, Q, \mu_{f}\right) \otimes \phi\left(x, \mu_{f}\right), \otimes \equiv \int_{0}^{1} d x
$$

where

$$
Q^{2}=-\frac{\left(q_{1}-q_{2}\right)^{2}}{4}, \quad \omega=\frac{q_{1}^{2}-q_{2}^{2}}{q_{1}^{2}+q_{2}^{2}} .
$$

In the above, the resolution scale $Q^{2}$ is large and the asymmetry parameter $\omega$ is fixed, i.e., $|\omega| \leqslant 1$, while $\mu_{f}$ represents the factorization scale. Because of Bose symmetry the transition form factor is symmetric in $\omega$. The perturbative expansion of the hard-scattering amplitude reads

$$
\begin{aligned}
T\left(\omega, x, Q, \mu_{f}\right)= & \frac{\sqrt{2}}{6 Q^{2}}\left[T^{(0)}(\omega, x)+\frac{\alpha_{s}\left(\mu_{r}\right)}{2 \pi} T^{(1)}\left(\omega, x, \frac{Q}{\mu_{f}}\right)\right. \\
& +\frac{\alpha_{s}^{2}\left(\mu_{r}\right)}{(2 \pi)^{2}} T^{(2)}\left(\omega, x, \frac{Q}{\mu_{f}}, \frac{Q}{\mu_{r}}\right) \\
& \left.+O\left(\alpha_{s}^{3}\right)+\{x \rightarrow 1-x\}\right],
\end{aligned}
$$

where $\mu_{r}$ is the renormalization scale and the leading-order (LO) contribution is given by

$$
T^{(0)}(\omega, x)=\frac{1}{1-\omega(2 x-1)} .
$$


The normalization of $T$ given above corresponds to $\phi\left(x, \mu_{f}\right)$ normalized to 1 and $f_{\pi}=131 \mathrm{MeV}$. Note that a residual dependence on the renormalization scale $\mu_{r}$ appears in the truncated perturbative expansion of the hard-scattering amplitude. The next-to-leading order (NLO) correction to the hard-scattering amplitude has been calculated in the modified minimal subtraction $(\overline{\mathrm{MS}})$ scheme [5-7]. In the next-tonext-to-leading order (NNLO), only the contributions coming from the quark-bubble insertions have been evaluated [8-10], again using the $\overline{\mathrm{MS}}$ scheme. The pion distribution amplitude $\phi\left(x, \mu_{f}\right)$ is intrinsically a nonperturbative quantity and cannot be determined from the perturbation theory. However, its evolution is governed by the evolution equation

$$
\mu_{f}^{2} \frac{d}{d \mu_{f}^{2}} \phi\left(x, \mu_{f}\right)=V\left(x, u, \mu_{f}\right) \otimes \phi\left(u, \mu_{f}\right),
$$

in which the evolution kernel has a perturbative expansion as

$$
V\left(x, y, \mu_{f}\right)=\frac{\alpha_{s}\left(\mu_{f}\right)}{2 \pi} V^{(0)}(x, y)+\frac{\alpha_{s}^{2}\left(\mu_{f}\right)}{(2 \pi)^{2}} V^{(1)}(x, y)+O\left(\alpha_{s}^{3}\right) .
$$

The evolution kernel has been estimated to NLO accuracy using the $\overline{\mathrm{MS}}$ scheme [11-13] and the corresponding solution of the evolution equation was obtained [14-16]. The latter can be expressed in the form

$$
\begin{aligned}
\phi\left(x, \mu_{f} \mid \mu_{0}\right)= & \phi^{(0)}\left(x, \mu_{f} \mid \mu_{0}\right)+\frac{\alpha_{s}\left(\mu_{f}\right)}{2 \pi} \phi^{(1)}\left(x, \mu_{f} \mid \mu_{0}\right) \\
& +O\left(\alpha_{s}^{2}\right),
\end{aligned}
$$

where the scale $\mu_{0}$ denotes some low scale at which the nonperturbative input was obtained. The solution (1.6) satisfies the initial condition $\phi\left(x, \mu_{0} \mid \mu_{0}\right)=\phi^{(0)}\left(x, \mu_{0} \mid \mu_{0}\right)$ and for $\mu_{f} \rightarrow \infty$ takes the asymptotic form $\phi\left(x, \mu_{f} \rightarrow \infty \mid \mu_{0}\right)$ $=6 x(1-x)$. We stress that the evolution equation as defined by (1.4) and (1.5) corresponds to the simplified scheme fixed by the preference that the distribution amplitude $\phi$ should have no residual dependence on the renormalization scale. ${ }^{1}$

The photon-to-pion transition form factor has been measured at large $Q^{2}$ by the CELLO [17] and CLEO [18] Collaborations, where one photon is almost on shell, while the second one has a virtuality up to $9 \mathrm{GeV}^{2}$. Different authors have analyzed the data [19-26], and it is often stated that the pion distribution amplitude is close to its asymptotic shape; for previous work, see also [27-29]. However, in this kinematics, the shape of the distribution amplitude can be con-

\footnotetext{
${ }^{1}$ Note that, in general, such a residual dependence appears along with the evolution kernel depending on two scales:

$$
\begin{aligned}
V\left(x, y, \mu_{f}\right)= & \frac{\alpha_{s}\left(\mu_{r}\right)}{2 \pi} V^{(0)}(x, y)+\frac{\alpha_{s}^{2}\left(\mu_{r}\right)}{(2 \pi)^{2}}\left[V^{(1)}(x, y)-\frac{\beta_{0}}{2} V^{(0)}(x, y) \ln \left(\frac{\mu_{r}^{2}}{\mu_{f}^{2}}\right)\right] \\
& +O\left(\alpha_{s}^{3}\right) .
\end{aligned}
$$
}

strained only from the scaling violation that arises from the evolution of the distribution amplitude. In the small $|\omega|$ region, perturbative QCD gives a parameter-free prediction of the photon-to-pion transition form factor, and in the intermediate region, one might extract the few lowest moments of the distribution amplitude and confront them with nonperturbative results (see Ref. [30]). However, both high-precision data as well as a precise understanding of perturbative and nonperturbative effects are necessary for this analysis.

Thus, the computation of both perturbative and power suppressed contributions is an important task. In this way, we can gain insight into the perturbative approach to exclusive processes. However, calculations of exclusive amplitudes beyond the leading order are quite cumbersome. In addition to the photon-to-meson transition form factor and similar exclusive two-photon processes, the perturbative next-to-leading order predictions are known only for the pion form factor [31-37] and for the amplitude of charged meson pair production in two-photon collisions for the case of equal momenta sharing meson distribution amplitude [38]. Fortunately, in the perturbative sector massless QCD is invariant under conformal transformation provided the coupling has a fixed point, so that the $\beta$ function, the renormalization group coefficient of the running coupling, vanishes. In the lowest order of $\alpha_{s}$, the conformal symmetry breaking part, which is consequently proportional to $\beta / g=\beta_{0} \alpha_{s} /(4 \pi)+O\left(\alpha_{s}^{2}\right)$, can be determined by calculating the Abelian part of the gluon self-energy proportional to the number of quarks $n_{f}$. Additional subtleties may appear owing to the factorization procedure and they can be resolved by a finite renormalization of the hard-scattering and distribution amplitudes. Making use of conformal symmetry constraints, together with the explicit calculation of terms proportional to the $\beta$ function, offers a considerable simplification of the perturbative calculation and, in our case, gives the possibility of going beyond the NLO approximation.

Indeed, for the photon-to-pion transition form factor we can take advantage of this symmetry and its predictive power $[39,40]$ by means of the conformal OPE (COPE) $[41,42]$, in which the form of the Wilson coefficients is constrained. The normalization of these coefficients can be recovered in the forward kinematics from the DIS results for the nonsinglet coefficient function of the polarized structure function $g_{1}$ known to NNLO [43]. This field-theoretical approach has been explored $[15,16,44-46]$ and tested to NLO [47], where the $\beta$ function is absent in the Wilson coefficients. We emphasise that the "conformal symmetry breaking" due to the factorization procedure in the $\overline{\mathrm{MS}}$ scheme and the restoration of conformal symmetry by finite renormalization are well understood at NLO. Further consistency checks are based on comparison with explicit results (e.g., hardscattering amplitudes for two-photon processes in the lightcone dominated region, the flavor nonsinglet kernel, quarkbubble insertions in singlet kernels), and with constraints coming from the $N=1$ super Yang-Mills theory [48].

In this paper we apply the COPE combining the NNLO result for the nonsinglet coefficient function of the polarized structure function $g_{1}$ [43] with the explicit result for the $n_{f^{-}}$ (i.e., $\beta_{0}$-)proportional NNLO contribution to the hard- 
scattering amplitude of the photon-to-pion transition form factor $[9,10]$, both being evaluated in the $\overline{\mathrm{MS}}$ scheme, to obtain a full NNLO result for the photon-to-pion transition form factor in the so-called conformal factorization scheme. Alternatively, we propose a scheme in which the hardscattering amplitude can already be constructed from a knowledge of the nonsinglet coefficient function of $g_{1}$ and the corresponding anomalous dimensions.

The paper is organized as follows. For the convenience of the reader, in Sec. II we review the predictive power of conformal symmetry relevant to the photon-to-pion transition form factor. We then propose two treatments of terms proportional to the $\beta$ function and discuss the remaining freedom in the choice of the factorization procedure. The general structure of the hard-scattering amplitude in the $\overline{\mathrm{MS}}$ scheme and the NNLO term that is proportional to $\beta_{0}$ are analyzed in Sec. III. For the phenomenologically important case in which one photon is quasireal, we evaluate conformal moments for the hard-scattering amplitude and by making use of the NNLO results for the $g_{1}$ function we obtain the NNLO prediction for the photon-to-pion transition form factor in the conformal factorization scheme. We extend this procedure to other values of photon virtualities and present a detailed investigation of the conformal partial wave decomposition of the transition form factor in different $\omega$ regions. Based on these results, in Sec. IV we analyze the size of the NLO and NNLO effects for one quasireal photon and in the small and intermediate $|\omega|$ regions. Finally, a summary and conclusions are given in Sec. V. The Appendixes are devoted to technical details: the Feynman-Schwinger representation of the hard-scattering amplitude, a consistency check at NNLO between the $n_{f}$-proportional $\overline{\mathrm{MS}}$ results for the hardscattering amplitude of the photon-to-pion transition form factor and the results for the nonsinglet coefficient function of the DIS polarized structure function $g_{1}$, evaluation of the conformal moments of the hard-scattering amplitude, the Taylor expansions in $\omega$, and the prescription for reconstructing the hard-scattering amplitude in the momentum fraction space from the known conformal moments.

\section{OUTLINING THE CONFORMAL SYMMETRY FORMALISM}

In the physical sector, massless QCD at the tree level is invariant under conformal transformations, i.e., under spacetime transformations containing the Poincaré transformations, dilatation, and special conformal transformations. The latter are composed of translation, inversion, and translation again. Conformal symmetry implies an improvement of the energy-momentum tensor, which then becomes traceless. Owing to this symmetry, one has additional constraints for field-theoretical quantities, e.g., for Green's functions. This subject was intensively studied in the 1960s and 1970s in four-dimensional field theory. In QCD, conformal symmetry is manifested in the Crewther relation [49] and in the solution for the mixing problem of composite operators under renormalization $[50,15,46]$. The reduced matrix elements of the conformal operators $\mathcal{O}$, sandwiched between the vacuum $|\Omega\rangle$ and one-pion $\langle\pi(P)|$ states, are pertinent to the expan- sion of the distribution amplitude ${ }^{2}$

$$
\begin{aligned}
\phi\left(x, \mu_{f}\right) & =\sum_{j=0}^{\infty} \frac{x(1-x)}{N_{j}} C_{j}^{3 / 2}(2 x-1)\left\langle\pi(P)\left|\mathcal{O}_{j j}\left(\mu_{f}\right)\right| \Omega\right\rangle^{\mathrm{red}}, \\
N_{j} & =\frac{(j+1)(j+2)}{4(2 j+3)} .
\end{aligned}
$$

Here $C_{j}^{3 / 2}$ are the Gegenbauer polynomials with index $\nu$ $=3 / 2$ of order $j$ and the sum runs over even $j$. In this representation, the transition form factor reads

$$
F_{\gamma \pi}(\omega, Q)=f_{\pi} \sum_{j=0}^{\infty} T_{j}\left(\omega, Q, \mu_{f}\right)\left\langle\pi(P)\left|\mathcal{O}_{j j}\left(\mu_{f}\right)\right| \Omega\right\rangle^{\mathrm{red}}
$$

where $T_{j}$ denotes the $j$ th conformal moment of the hardscattering amplitude:

$$
\begin{aligned}
T_{j}\left(\omega, Q, \mu_{f}\right)= & \int_{0}^{1} d x T\left(\omega, x, Q, \mu_{f}\right) \frac{x(1-x)}{N_{j}} C_{j}^{3 / 2}(2 x-1) \\
= & \frac{\sqrt{2}}{3 Q^{2}}\left[T_{j}^{(0)}(\omega)+\frac{\alpha_{s}\left(\mu_{r}\right)}{2 \pi} T_{j}^{(1)}\left(\omega, \frac{Q}{\mu_{f}}\right)\right. \\
& \left.+\frac{\alpha_{s}^{2}\left(\mu_{r}\right)}{(2 \pi)^{2}} T_{j}^{(2)}\left(\omega, \frac{Q}{\mu_{f}}, \frac{Q}{\mu_{r}}\right)+O\left(\alpha_{s}^{3}\right)\right] .
\end{aligned}
$$

As reviewed in Sec. II A, the operator mixing problem under renormalization beyond the one-loop level is solved by the restoration of conformal symmetry. In Sec. II B this allows us to employ conformal symmetry in the OPE of two electromagnetic currents, and to fix the hard-scattering amplitude (2.3) in the conformal limit. Additionally, we include $\beta$-proportional terms and in Sec. II C we discuss the corresponding ambiguities of this procedure. The solution of the renormalization group equation to NNLO is worked out in Sec. II D.

\section{A. Renormalization properties of conformal operators and the conformal scheme}

Let us start with the constraints for the renormalization of composite operators. In the flavor nonsinglet and parity odd sector the twist-2 operators read [51-53]

\footnotetext{
${ }^{2}$ It is common in the literature that the distribution amplitude is expanded in the form

$$
\phi\left(x, \mu_{f}\right)=6 \sum_{j=0}^{\infty} x(1-x) B_{j}\left(\mu_{f}\right) C_{j}^{3 / 2}(2 x-1),
$$

where $B_{j}(j=2,4, \ldots)$ essentially represent the nonperturbative input. Comparing with our definition (2.1), we have $\left\langle\pi(P)\left|\mathcal{O}_{j j}\left(\mu_{f}\right)\right| \Omega\right\rangle^{\text {red }}=6 N_{j} B_{j}\left(\mu_{f}\right)$, where $\left\langle\pi(P)\left|\mathcal{O}_{00}\left(\mu_{f}\right)\right| \Omega\right\rangle^{\text {red }}$ $=B_{0}=1$ is a renormalization group invariant quantity.
} 


$$
\mathcal{O}_{j l}=\left.\bar{\psi}(x)(n \cdot \gamma) \gamma_{5} C_{j}^{3 / 2}\left(\frac{n \cdot \overleftrightarrow{D}}{n \cdot \partial}\right)(i n \cdot \partial)^{l} \psi(y)\right|_{x=y=0},
$$

where $\partial=\vec{\partial}+\overleftarrow{\partial}, \overleftrightarrow{D}=\vec{D}-\overleftarrow{D}$, and $n$ is a lightlike vector that makes these operators symmetric and traceless. The Gegenbauer polynomials $C_{j}^{3 / 2}$ arise from the group-theoretical construction of the operators and they are of order $j$, where this label is related to the conformal spin $j+1$, i.e., the eigenvalue of the Casimir operator of the so-called collinear conformal group. These operators have spin $l+1$ and canonical dimension $l+3$. In other words, we have different infinite irreducible representations of the conformal algebra, called towers, that are characterized by the conformal spin $j+1$, while the members of each representation are labeled by the spin $l+1$. The conformal operators with $l=j$ are the lowest members of each conformal tower, and we can climb the tower by acting with the generator of translation.

Employing Poincare invariance, the general form of the renormalization group equation for the operators introduced above reads

$$
\mu \frac{d}{d \mu} \mathcal{O}_{j l}=-\sum_{k=0}^{j} \gamma_{j k} \mathcal{O}_{k l}
$$

In the conformally invariant theory operators of different conformal towers do not mix under renormalization. Indeed, the anomalous dimension matrix

$$
\begin{aligned}
\gamma_{j k}= & \frac{\alpha_{s}}{2 \pi} \delta_{j k} \gamma_{j}^{(0)}+\frac{\alpha_{s}^{2}}{(2 \pi)^{2}} \gamma_{j k}^{(1)}+\frac{\alpha_{s}^{3}}{(2 \pi)^{3}} \gamma_{j k}^{(2)} \\
& +O\left(\alpha_{s}^{4}\right) \quad \text { with } \quad \gamma_{j} \equiv \gamma_{j j}
\end{aligned}
$$

is diagonal at LO. This property is induced by conformal symmetry at the tree level. The fact that these operators will mix beyond $\mathrm{LO}$ even for the vanishing $\beta$ function in the $\overline{\mathrm{MS}}$ scheme, has been considered as an unexpected breakdown of conformal symmetry. Note that the appearance of the anomalous dimension already requires a "redefinition" of the conformal representation at the tree level, i.e., the scaling dimensions of the operators change.
The understanding of this subtlety is the key for the application of conformal symmetry in all orders of perturbation theory. It is well known that, on the quantum level, conformal symmetry is broken owing to the regularization of ultraviolet divergences, which shows up in the trace anomaly of the (improved) energy-momentum tensor. This trace anomaly is given as a linear combination of different renormalized operators. In the dimensionally regularized theory with space-time dimension $n=4-2 \epsilon$, it reads

$$
\Theta_{\mu \mu}(x)=\frac{\beta_{\epsilon}}{2 g}\left(G_{\mu \nu}^{a}(x)\right)^{2}+\cdots,
$$

where the $\beta$ function in the regularized theory is defined as

$$
\beta_{\epsilon} \equiv \mu \frac{\partial g}{\partial \mu}=-\frac{4-n}{2} g+\beta \quad \text { with } \quad \frac{\beta}{g}=\frac{\alpha_{s}}{4 \pi} \beta_{0}+O\left(\alpha_{s}^{2}\right)
$$

and $\beta_{0}=(2 / 3) n_{f}-(11 / 3) C_{A}$. In addition to the square of the renormalized field strength tensor $G_{\mu \nu}^{a}$ multiplied by the $\beta$ function, the trace anomaly (2.7) contains equations of motion and BRST-exact operators. Therefore, it is sometimes believed that in the physical sector of the theory the breaking of conformal symmetry is in general proportional to the $\beta$ function. However, if one deals with composite operators, the operator product and the trace anomaly of these operators contain additional ultraviolet divergences. Since they are multiplied by the $(4-n)$ contribution in $\beta_{\epsilon}$, these UV divergences produce finite symmetry breaking terms that are not proportional to the $\beta$ function. The appearance of anomalous dimensions of composite operators can also be understood in this way.

A detailed analysis shows that the nondiagonality of the anomalous dimension matrix observed in the $\overline{\mathrm{MS}}$ scheme at NLO originates from such an effect of conformal symmetry breaking. It already appears at LO in the Ward identities of these operators with respect to the special conformal transformation. The calculation of this special conformal anomaly matrix $\hat{\gamma}^{c}(l)=\left(\alpha_{s} / 2 \pi\right) \hat{\gamma}^{c(0)}(l)+O\left(\alpha_{s}^{2}\right)$ results in

$$
\hat{\gamma}^{c(0)}(l)=-\hat{b}(l) \hat{\gamma}^{(0)}+\hat{w},
$$

where

$$
b_{j k}(l)=\left\{\begin{array}{cc}
2(l+k+3) \delta_{j k}-2(2 k+3), & j-k \geqslant 0 \text { and even } \\
0 & \text { otherwise }
\end{array}\right.
$$

and

$$
\begin{aligned}
& w_{j k}=C_{F}\left\{\begin{array}{c}
-4(2 k+3)(j-k)(j+k+3)\left[\frac{A_{j k}-\psi(j+2)+\psi(1)}{(k+1)(k+2)}+\frac{2 A_{j k}}{(j-k)(j+k+3)}\right], \quad j-k>0 \text { and even, } \\
0
\end{array}\right. \\
& A_{j k}=\psi\left(\frac{j+k+4}{2}\right)-\psi\left(\frac{j-k}{2}\right)+2 \psi(j-k)-\psi(j+2)-\psi(1),
\end{aligned}
$$


with $\psi(z)=(d / d z) \ln \Gamma(z)$ and $C_{F}=4 / 3$. It induces offdiagonal entries in the anomalous dimension matrix (2.6):

$$
\gamma_{j k}^{\overline{\mathrm{MS}}(1)}=-\frac{\left[\hat{\gamma}^{c(0)}+\beta_{0} \hat{b}, \gamma^{(0)}\right]_{j k}}{a(j, k)} \text { for } j>k,
$$

where $a(j, k)=2(j-k)(j+k+3)$. The prediction (2.11) coincides with the moments of the explicitly calculated evolution kernel at NLO [11-13]. On the other hand, the diagonal part of the anomalous dimension matrix (2.6)

$$
\gamma_{j j} \equiv \gamma_{j}=\frac{\alpha_{s}}{2 \pi} \gamma_{j}^{(0)}+\frac{\alpha_{s}^{2}}{(2 \pi)^{2}} \gamma_{j}^{(1)}+\frac{\alpha_{s}^{3}}{(2 \pi)^{3}} \gamma_{j}^{(2)}+O\left(\alpha_{s}^{4}\right)
$$

coincides with the anomalous dimensions of the operators that are restricted to the forward kinematics and are known as the moments of the splitting kernels in DIS.

As we can see, there is a complete understanding of conformal symmetry breaking in the $\overline{\mathrm{MS}}$ scheme. Now the questions arise: Can we find a scheme at which conformal symmetry holds true? Can we then use the predictive power of conformal symmetry?

The first question has a positive answer in the case that the $\beta$ function has a fixed point. Instead of relying on the hypothetical fixed point, we simply freeze the coupling by hand, which implies $\beta=0$. It is then possible to find a scheme in which the renormalized conformal operators (2.4) form an irreducible representation of the collinear conformal group, i.e., their special conformal anomaly and anomalous dimension matrices are simultaneously diagonal. The rotation from the $\overline{\mathrm{MS}}$ to such a scheme, which we call the conformal subtraction (CS) scheme, is given by the matrix $\hat{B}$ defined by

$$
\mathcal{O}^{\mathrm{CS}}=\hat{B}^{-1} \mathcal{O}^{\overline{\mathrm{MS}}}, \quad B_{j k}=\delta_{j k}+\frac{\alpha_{s}}{2 \pi} B_{j k}^{(1)}+O\left(\alpha_{s}^{2}\right) .
$$

The NLO term $B_{j k}^{(1)}$ is entirely determined by the special conformal anomaly (2.9) and reads

$$
B_{j k}^{(1)}=-\theta(j>k) \frac{\gamma_{j k}^{c(0)}}{a(j, k)}=\theta(j>k)\left\{\hat{d} \hat{\gamma}^{(0)}-\hat{g}\right\}_{j k} .
$$

Here we introduce the notation $d_{j k}=b_{j k} / a(j, k)$ and $g_{j k}$ $=w_{j k} / a(j, k)$. In the case of a nonvanishing $\beta$ function, an additional off-diagonal term appears, and thus the complete anomalous dimension in the CS scheme reads $[15,16]$

$$
\begin{aligned}
\gamma_{j k}^{\mathrm{CS}} & =\left\{\hat{B}^{-1} \hat{\gamma}^{\overline{\mathrm{MS}}} \hat{B}+\hat{B}^{-1}\left[\mu \frac{d}{d \mu} \hat{B}\right]\right\}_{j k} \\
& =\delta_{j k} \gamma_{j}+\theta(j>k) \frac{\beta}{g} \Delta_{j k} .
\end{aligned}
$$

The addendum of the anomalous dimension matrix (2.15) is known in the lowest order of $\alpha_{s}$ [40]:

$$
\begin{aligned}
\Delta_{j k} & =\frac{\alpha_{s}}{2 \pi} \Delta_{j k}^{(0)}+\frac{\alpha_{s}^{2}}{(2 \pi)^{2}} \Delta_{j k}^{(1)}+O\left(\alpha_{s}^{3}\right) \text { with } \\
\Delta_{j k}^{(0)} & =B_{j k}^{(1)}-\left[\hat{d}, \hat{\gamma}^{(0)}\right]_{j k} .
\end{aligned}
$$

Note that the diagonal part (2.12), which corresponds to the moments of the splitting kernels, also includes $\beta$-proportional terms. The moments of the splitting kernels are completely known to two-loop accuracy [54-57] and the first 13 entries at three-loop level are given in [58]. Their scheme dependence is fixed by the fact that they are evaluated in the $\overline{\mathrm{MS}}$ scheme. This implies that the diagonal part of $\hat{B}$ is given by the identity matrix.

\section{B. Conformal operator product expansion}

Let us now turn to the second question we have raised. As we discussed in the preceding subsection, one advantage of the conformal scheme is that, up to off-diagonal terms proportional to $\beta$, the anomalous dimensions are fixed by the DIS results, and, in the conformal limit, they are partly known to NNLO order. ${ }^{3}$ Furthermore, the class of twophoton processes that are light-cone dominated, i.e., for $Q^{2}$ large, can be treated by means of OPE. That includes the evaluation of the corresponding nonforward Wilson coefficients. Under the assumption that conformal symmetry holds true, these coefficients are fixed up to normalization factors that coincide with the Wilson coefficients appearing in the deep inelastic scattering structure functions $F_{1}$ and $g_{1}$ $[41,42]$. Hence, in the conformal scheme, taking the conformal limit in which conformal symmetry holds true, we can use this predictive power of the COPE to avoid cumbersome higher-order calculations. Indeed, the NLO coefficient functions for the hadronic tensor in the general off-forward kinematics were predicted in this way [44] and they coincide, after rotation to the $\overline{\mathrm{MS}}$ scheme, with explicitly calculated ones [60-62].

For the photon-to-pion transition form factor, the leading twist-2 result of OPE is given by Eq. (2.2), where the conformal moments $T_{j}$ of the hard-scattering amplitude correspond to the Wilson coefficients $C_{j}$, which are conventionally normalized as

$$
T_{j}(\omega, Q, \mu)=\frac{\sqrt{2}}{3 Q^{2}} C_{j}\left(\omega \mid \alpha_{s}(\mu), Q / \mu\right) .
$$

As we have mentioned, in the formal conformal limit the Wilson coefficients are constrained in the CS scheme by the predictive power of the COPE:

$$
\alpha_{s}(\mu) \Rightarrow \alpha_{s}^{*}-\text { fixed }
$$

implies the reduction

\footnotetext{
${ }^{3}$ In principle, we then also know the Efremov-RadyushkinBrodsky-Lepage (ERBL) evolution kernels, which can be obtained from the Dokshitzer-Gribov-Lipatov-Altarelli-Parisi (DGLAP) kernels through an integral transformation [59].
} 


$$
C_{j}\left(\omega \mid \alpha_{s}(\mu), Q / \mu\right) \Rightarrow \bar{C}_{j}\left(\omega \mid \alpha_{s}^{*}, Q / \mu\right),
$$

where $\bar{C}_{j}\left(\omega \mid \alpha_{s}^{*}, Q / \mu\right)$ is given by [39]

$$
\begin{aligned}
\bar{C}_{j}= & c_{j}\left(\alpha_{s}^{*}\right)\left(\frac{\mu^{2}}{Q^{2}}\right)^{\gamma_{j} / 2} \frac{2(2 \omega)^{j}}{(1+\eta \omega)^{j+1+\gamma_{j} / 2}} B(j+1, j+2) \\
& \times{ }_{2} F_{1}\left(\begin{array}{c}
j+1+\frac{1}{2} \gamma_{j}, j+2+\frac{1}{2} \gamma_{j} \\
2\left(j+2+\frac{1}{2} \gamma_{j}\right)
\end{array} \mid \frac{2 \eta \omega}{1+\eta \omega}\right) .
\end{aligned}
$$

In the limit $\eta \rightarrow 1$, one obtains the result for the production of a (pseudo)scalar meson by two virtual photons, while for $\eta=0$ the forward case is reproduced. ${ }^{4}$ Note that $C_{j}(\omega)$ is an even (odd) function of $\omega$ for even (odd) $j$, which is guaranteed by the linear transformation properties of the hypergeometric functions ${ }_{2} F_{1}$. The normalization $c_{j}\left(\alpha_{s}\right)$ coincides with the flavor nonsinglet Wilson coefficients of the polarized structure function $g_{1}$ taken at $\mu=Q$. It is given as a perturbative expansion:

$c_{j}\left(\alpha_{s}\right)=c_{j}^{(0)}+\frac{\alpha_{s}}{2 \pi} c_{j}^{(1)}+\frac{\alpha_{s}^{2}}{(2 \pi)^{2}} c_{j}^{(2)}+O\left(\alpha_{s}^{3}\right) \quad$ with $c_{j}^{(0)}=1$,

and is known to NNLO in the $\overline{\mathrm{MS}}$ scheme [43]. Strictly speaking, this coincidence appears just at the hypothetical fixed point $\alpha_{s}=\alpha_{s}^{*}$. However, since we know the forward anomalous dimensions and the Wilson coefficients perturbatively in the $\overline{\mathrm{MS}}$ scheme, we can easily restore $\beta$-proportional terms in these quantities beyond the conformal limit.

Conformal symmetry breaking terms proportional to the $\beta$ function alter the COPE result (2.18) in the full theory. Obviously, in the irreducible conformal representation used, the $\beta$ term cannot be fixed from the requirement of conformal invariance. Thus, the definition of the conformal scheme

$$
C^{\mathrm{CS}}\left(\alpha_{s}(\mu), Q / \mu\right)=C^{\overline{\mathrm{MS}}}\left(\alpha_{s}(\mu), Q / \mu\right) \hat{B}\left(\alpha_{s}(\mu)\right)
$$

with

$$
C^{\mathrm{CS}}\left(\alpha_{s}^{*}, Q / \mu\right)=\bar{C}\left(\alpha_{s}^{*}, Q / \mu\right)
$$

is ambiguous and, consequently, the $\hat{B}$ matrix is uniquely defined only up to $\beta$-proportional terms that are off diagonal. At the two-loop level, $C$ contains $\beta_{0}$-proportional terms ap-

\footnotetext{
${ }^{4}$ The Wilson coefficients appearing in the deep inelastic scattering structure functions $F_{1}$ and $g_{1}$ are derived in the usual DIS operator basis, which differs in the normalization from the definition for the basis of conformal operators. Hence, these Wilson coefficients differ slightly from the $\eta=0$ limit of Eq. (2.18).
}

pearing in both $c_{j}^{(2)}$ and $\gamma_{j}^{(2)}$. Let us first set such terms to zero and, with the help of this, single out all $\beta_{0}$-proportional terms in $C^{\mathrm{CS}}$ :

$$
\begin{aligned}
C^{\mathrm{CS}} & =\left.C^{\overline{\mathrm{MS}}} \hat{B}\right|_{\beta=0}+\frac{\beta}{g} \delta C \\
& =\left.\bar{C}\right|_{\beta=0}+\frac{\beta}{g} \delta C \quad \text { with } \\
\delta C & =\frac{g}{\beta}\left(C^{\overline{\mathrm{MS}}} \hat{B}-\left.C^{\overline{\mathrm{MS}}} \hat{B}\right|_{\beta=0}\right),
\end{aligned}
$$

and $\alpha_{s}$ remains running. At $\mathrm{LO}$ a $\beta_{0}$ term does not appear in $C^{\overline{\mathrm{MS}}}$; thus, the perturbative expansion

$$
\delta C_{j}=\delta C_{j}^{(0)}+\frac{\alpha_{s}}{2 \pi} \delta C_{j}^{(1)}+O\left(\alpha_{s}^{2}\right)
$$

holds true. Note that since $B_{j k}^{(0)}=\delta_{j k}$ [Eq. (2.13)] the LO coefficient $C^{\overline{\mathrm{MS}}(0)} \equiv C^{(0)}$ is independent of the scheme. Similarly to Eq. (2.21), we can write the matrix $\hat{B}$ in the general form

$$
\begin{gathered}
\hat{B}=\left.\hat{B}\right|_{\beta=0}+\frac{\beta}{g} \delta \hat{B} \quad \text { with } \\
\delta \hat{B}=\delta \hat{B}^{(0)}+\frac{\alpha_{s}}{2 \pi} \delta \hat{B}^{(1)}+O\left(\alpha_{s}^{2}\right) .
\end{gathered}
$$

If we define $\hat{B}$ so that it contains no $\beta_{0}$ term at order $\alpha_{s}$, i.e., if we take $\delta \hat{B}^{(0)}=0$ as in our definition of the CS scheme (2.13), (2.14), the coefficients $\delta C$ up to NNLO read

$$
\delta C^{(0)}=0, \quad \delta C^{(1)}=\frac{2}{\beta_{0}}\left(C^{\overline{\mathrm{MS}}(2)}-\left.C^{\overline{\mathrm{MS}}(2)}\right|_{\beta_{0}=0}\right)+C^{(0)} \delta \hat{B}^{(1)} .
$$

Since we have required that the diagonal entries of $\hat{B}$ should be 1 , the normalization coefficients $c_{j}$ coincide in the forward limit with the Wilson coefficients of $g_{1}$ calculated in the $\overline{\mathrm{MS}}$ scheme.

\section{Ambiguities in the definition of the conformal scheme}

As we have discussed, the ambiguity left in the definition of the conformal scheme in the full theory resides in the $\beta$-proportional off-diagonal terms, i.e., in the choice of $\delta \hat{B}$ in Eq. (2.23). Adopting the definitions (2.13) and (2.23), we set $\delta \hat{B}^{(0)}=0$ and in the following discuss different choices of $\delta \hat{B}^{(1)}$, restricting ourselves to NNLO.

\section{Defining CS and $\overline{C S}$ schemes}

The naive choice is to set

$$
\delta \hat{B}^{(1)}=0,
$$


which means that the $\beta_{0}$-proportional term in the NNLO Wilson coefficients, i.e., $\delta C^{(1)}$, is entirely evaluated in the $\overline{\mathrm{MS}}$ scheme. Since the conformal symmetry breaking part appearing in the COPE has to be proportional to the $\beta$ function, we can calculate $\delta C^{(1)}$ by evaluating the contributions proportional to the $n_{f}$ piece of $\beta_{0}$, i.e., from the one-loop Feynman graphs with an additional quark-bubble insertion.

In this naive scheme (2.25), which we denote by CS, both the conformal operators and the Wilson coefficients will mix under renormalization to NLO accuracy owing to the running of the coupling. Let us consider this in more detail. Since the transition form factor is invariant under renormalization, i.e.,

$$
\mu \frac{d}{d \mu} F_{\gamma \pi}(\omega, Q)=0
$$

these effects will compensate one another. Thus, the renormalization group equation for the Wilson coefficients reads

$$
\begin{aligned}
{\left[\mu \frac{\partial}{\partial \mu}+\beta \frac{\partial}{\partial g}\right] C_{j}^{\mathrm{CS}}=} & {\left[\frac{\alpha_{s}(\mu)}{2 \pi} \gamma_{j}^{(0)}+\frac{\alpha_{s}^{2}(\mu)}{(2 \pi)^{2}} \gamma_{j}^{(1)}\right] C_{j}^{\mathrm{CS}} } \\
& +\frac{\beta}{g} \sum_{i=j+2}^{\infty} \frac{\alpha_{s}(\mu)}{2 \pi} C_{i}^{\mathrm{CS}} \Delta_{i j}^{(0)} \\
& +O\left(\alpha_{s}^{3}\right),
\end{aligned}
$$

where the addendum $\Delta_{i j}^{(0)}$ is defined in Eq. (2.16). Since $C_{j}^{\mathrm{CS}}$ is conformally covariant to the NLO approximation, i.e., it contains no partial waves (2.18) with a conformal spin larger than $j+1$, the off-diagonal entries on the right-hand side (RHS) arise from the explicit $\mu$ dependence of $C_{j}^{\mathrm{CS}(2)}$, which has been taken from the $\overline{\mathrm{MS}}$ scheme.

In the alternative conformal scheme, denoted in the following by $\overline{\mathrm{CS}}$, this intermediate mixing is avoided by the complete diagonalization of the renormalization group equation. This can be achieved by including an explicit $\mu$ dependence in the $\hat{B}$ matrix, i.e., by taking

$$
\delta B_{j k}^{(1)}=\ln \left(\frac{\mu^{* 2}}{\mu^{2}}\right) \Delta_{j k}^{(0)} \theta(j>k)+\cdots .
$$

In the order we are considering, the matrix $\hat{B}$ now reads

$$
\begin{aligned}
B_{j k}= & \delta_{j k}+\frac{\alpha_{s}(\mu)}{2 \pi} B_{j k}^{(1)}+\frac{\alpha_{s}^{2}(\mu)}{(2 \pi)^{2}}\left\{B_{j k}^{(2)}+\frac{\beta_{0}}{2}\right. \\
& \left.\times\left[\ln \left(\frac{\mu^{* 2}}{\mu^{2}}\right) \Delta_{j k}^{(0)} \theta(j>k)+\cdots\right]\right\}+O\left(\alpha_{s}^{3}\right) .
\end{aligned}
$$

This choice introduces $\gamma^{\overline{\mathrm{CS}}}$ given by Eqs. (2.15), (2.16) with $\Delta_{j k}^{(0)} \rightarrow \Delta_{j k}^{\overline{C S}(0)}=0$, i.e., the LO addendum to the anomalous dimension matrix vanishes in this scheme. However, for dimensional reasons the choice (2.29) additionally introduces a new residual scale dependence $\mu^{*}$. The meaning of this procedure is obvious. We do not resum the remaining offdiagonal $\ln \mu$ terms through the renormalization group equation; rather, we include them in the Wilson coefficients, where they will be annulled. This is indeed on the same footing with what we have already discussed in the $\beta=0$ case, where the off-diagonal entries present in the $\overline{\mathrm{MS}}$ scheme have been removed by a finite, however, $\mu$ independent renormalization. Consequently, both the operators and the Wilson coefficients

$$
\mathcal{O}^{\overline{\mathrm{CS}}}=\hat{B}^{-1} \mathcal{O}^{\overline{\mathrm{MS}}}, \quad C^{\overline{\mathrm{CS}}}=C^{\overline{\mathrm{MS}}} \hat{B}
$$

where $\hat{B}$ is defined by Eq. (2.29), now satisfy the desired renormalization group equations in the $\alpha_{s}^{2}$ approximation:

$$
\begin{aligned}
\mu \frac{d}{d \mu} \mathcal{O}_{j l}^{\overline{\mathrm{CS}}}(\mu)=-\left[\frac{\alpha_{s}(\mu)}{2 \pi} \gamma_{j}^{(0)}+\frac{\alpha_{s}^{2}(\mu)}{(2 \pi)^{2}} \gamma_{j}^{(1)}\right. \\
\left.+O\left(\alpha_{s}^{3}\right)\right] \mathcal{O}_{j l}^{\overline{\mathrm{CS}}}(\mu), \\
{\left[\mu \frac{\partial}{\partial \mu}+\beta \frac{\partial}{\partial g}\right] C_{j}^{\overline{\mathrm{CS}}}\left(\alpha_{s}(\mu), Q / \mu\right) } \\
=\left[\frac{\alpha_{s}(\mu)}{2 \pi} \gamma_{j}^{(0)}+\frac{\alpha_{s}^{2}(\mu)}{(2 \pi)^{2}} \gamma_{j}^{(1)}+O\left(\alpha_{s}^{3}\right)\right] \\
\times C_{j}^{\overline{\mathrm{CS}}}\left(\alpha_{s}(\mu), Q / \mu\right) .
\end{aligned}
$$

Note that the forward anomalous dimensions $\gamma_{j}$ remain explicitly $\mu$ independent. However, both the off-diagonal piece of the anomalous dimensions and the Wilson coefficients now possess a residual $\mu^{*}$ dependence at the orders $\alpha_{s}^{3}$ and $\alpha_{s}^{2}$, respectively.

To restore the $\mu$ dependence of the Wilson coefficient, we perturbatively solve its renormalization group equation (2.32). Up to an integration constant $\delta C^{\prime}$, its solution to two-loop accuracy can be expressed by the Wilson coefficient (2.18), appearing in the COPE,

$$
C_{j}^{\overline{\mathrm{CS}}}\left(\alpha_{s}(\mu), Q / \mu\right)=\bar{C}_{j}\left(\alpha_{s}(\mu), Q / \mu\right)+\frac{\alpha_{s}^{2}}{(2 \pi)^{2}} \frac{\beta_{0}}{2} \delta C^{\prime},
$$

which now depend on the running coupling:

$$
\begin{aligned}
& \bar{C}_{j}=c_{j}\left(\alpha_{s}(\mu), \frac{Q}{\mu}, \frac{\partial}{\partial \gamma_{j}}\right)\left(\frac{\mu^{2}}{Q^{2}}\right)^{\gamma_{j} / 2} \frac{2(2 \omega)^{j} B(j+1, j+2)}{(1+\omega)^{j+1+\gamma_{j} / 2}} \\
& \times{ }_{2} F_{1}\left(\begin{array}{c|c}
j+1+\frac{1}{2} \gamma_{j}, j+2+\frac{1}{2} \gamma_{j} & \frac{2 \omega}{1+\omega} \\
2\left(j+2+\frac{1}{2} \gamma_{j}\right) &
\end{array}\right) .
\end{aligned}
$$


The running of $\alpha_{s}(\mu)$, appearing in the lowest approximation of both $\gamma_{j}$ and $c_{j}$, is compensated by introducing a logarithmic change of the $\mu$ dependence in the normalization factors:

$$
\begin{aligned}
c_{j}\left(\alpha_{s}(\mu), \frac{Q}{\mu}, \frac{\partial}{\partial \gamma_{j}}\right)= & c_{j}^{(0)}+\frac{\alpha_{s}(\mu)}{2 \pi} c_{j}^{(1)}+\frac{\alpha_{s}^{2}(\mu)}{(2 \pi)^{2}} c_{j}^{(2)}+\frac{\alpha_{s}(\mu)}{2 \pi} \frac{\beta_{0}}{2} \ln \left(\frac{Q^{2}}{\mu^{2}}\right) \\
& \times\left\{\frac{\alpha_{s}(\mu)}{2 \pi}\left[c_{j}^{(1)}+c_{j}^{(0)} \frac{\gamma_{j}^{(0)}}{4} \ln \left(\frac{Q^{2}}{\mu^{2}}\right)\right]+c_{j}^{(0)} \gamma_{j}^{(0)} \frac{\partial}{\partial \gamma_{j}^{(0)}}\right\}+O\left(\alpha_{s}^{3}\right) .
\end{aligned}
$$

Equation (2.34) should be understood in the sense of a consequent expansion with respect to $\alpha_{s}$ up to order $\alpha_{s}^{2}$.

So far we have found a rather natural way to include the effects of the running coupling in the structure of the COPE result, with the advantage that the conformal operators do not mix under renormalization in NLO. It remains to fix the integration constant $\delta C^{\prime}$, which vanishes in the kinematical forward limit. We can identify it with the noncovariant part calculated in the $\overline{\mathrm{MS}}$ scheme, in an analogous way to our discussion in the case of the CS scheme. On the other hand, it is rather appealing that the Wilson coefficients $C_{j}\left(\alpha_{s}(\mu), Q^{2} / \mu^{2}\right)$ contain only conformally covariant terms to NNLO. For the scheme we call $\overline{\mathrm{CS}}$, we adopt this prescription, i.e., we put $\delta C^{\prime}=0$. In the NNLO approximation, we then have a partial wave decomposition of the transition form factor with respect to the "good" quantum numberconformal spin. This in principle allows us to extract the conformal moments of the distribution amplitude with a well-defined conformal spin for experimental data.

\section{Calculational prescriptions}

Let us comment on the renormalization scale dependence and give the calculational prescription for the schemes we have proposed.

First, we introduced a naive recipe (CS scheme) which combines the COPE result with the one explicitly calculated in the $\overline{\mathrm{MS}}$ scheme. Now we extend our analysis by distinguishing between the renormalization scale $\mu_{r}$ (the argument of $\alpha_{s}$ in the Wilson coefficients) and the factorization scale $\mu_{f}$. We require that the matrix elements of conformal operators should depend only on the factorization scale $\mu_{f}$. Thus, the scheme transformation now reads

$$
\begin{aligned}
C^{\mathrm{CS}} & \left(\alpha_{s}\left(\mu_{r}\right), Q / \mu_{f}, Q / \mu_{r}\right) \\
& =C^{\overline{\mathrm{MS}}}\left(\alpha_{s}\left(\mu_{r}\right), Q / \mu_{f}, Q / \mu_{r}\right) \hat{B}\left(\alpha_{s}\left(\mu_{f}\right)\right) .
\end{aligned}
$$

Employing the scale-changing relation

$$
\alpha_{s}\left(\mu_{f}\right)=\alpha_{s}\left(\mu_{r}\right)\left[1+\frac{\alpha_{s}\left(\mu_{r}\right)}{2 \pi} \frac{\beta_{0}}{2} \ln \left(\frac{\mu_{f}^{2}}{\mu_{r}^{2}}\right)+O\left(\alpha_{s}^{2}\right)\right],
$$

we expand the rotation matrix

$$
\hat{B}\left(\alpha_{s}\left(\mu_{f}\right)\right)=\hat{B}\left(\alpha_{s}\left(\mu_{r}\right)\right)+\frac{\alpha_{s}^{2}\left(\mu_{r}\right)}{(2 \pi)^{2}} \frac{\beta_{0}}{2} \ln \left(\frac{\mu_{f}^{2}}{\mu_{r}^{2}}\right) \hat{B}^{(1)}+O\left(\alpha_{s}^{3}\right)
$$

Hence, in this scheme, the Wilson coefficients read to NNLO accuracy

$$
C^{\mathrm{CS}}=\left.\bar{C}\left(\alpha_{s}\left(\mu_{r}\right), Q / \mu_{f}\right)\right|_{\beta=0}+\frac{\beta_{0}}{2} \frac{\alpha_{s}^{2}\left(\mu_{r}\right)}{(2 \pi)^{2}}\left[-C_{\beta}^{\overline{\mathrm{MS}}(2)}\left(\alpha_{s}\left(\mu_{r}\right), Q / \mu_{f}, Q / \mu_{r}\right)+\ln \left(\frac{\mu_{f}^{2}}{\mu_{r}^{2}}\right) C^{(0)} \hat{B}^{(1)}\right]+O\left(\alpha_{s}^{3}\right)
$$

where $C_{\beta}^{\overline{\mathrm{MS}}(2)}$ denotes the $\left(-\beta_{0} / 2\right)$-proportional contribution evaluated in the $\overline{\mathrm{MS}}$ scheme, while the structure of $\bar{C}$ is fixed by Eqs. (2.18) and (2.19).

Alternatively, in the CS scheme, we employed renormalization group invariance to incorporate the running of the coupling into the generic structure of the COPE result and used a finite renormalization to preserve the structure of the COPE to NNLO accuracy:

$$
C_{j}^{\overline{\mathrm{CS}}}\left(\alpha_{s}(\mu), Q / \mu\right)=\bar{C}_{j}\left(\alpha_{s}(\mu), Q / \mu\right),
$$

where $\bar{C}_{j}$ is defined by Eqs. (2.34) and (2.35). The form of the Wilson coefficients in which the distinction between the scales $\mu_{r}$ and $\mu_{f}$ is introduced can be obtained analogously to the previously discussed case of the CS scheme, and will be presented in Sec. III C.

In Sec. IV we employ both of these schemes to estimate the size of NNLO effects at a given input scale. The missing ingredient for a consistent NNLO analysis including the evolution of the distribution amplitude is the anomalous dimension matrix at the three-loop level. Whereas the first few diagonal entries have been calculated, the off-diagonal part is 
unknown. It could be read off from the $n_{f}$-proportional part of the special conformal anomaly matrix in the two-loop approximation. Also, generally, the trace anomaly will affect the COPE at NNLO accuracy [see Eq. (2.7)]. We rather make use of the freedom to rotate the conformal symmetry breaking piece from the perturbative sector to the nonperturbative one, as has been done in the $\overline{\mathrm{CS}}$ scheme, or the reverse. However, we expect that the mixing effect in the $\overline{\mathrm{CS}}$ scheme will be negligibly small and its detailed investigation is beyond the scope of this paper.

\section{Evolutional behavior of conformal operators}

We end this section with a short review of the evolutional behavior of the conformal operators from which that of the distribution amplitude $\phi(x, \mu)$ can easily be established. For the convenience of the reader, we repeat here the basic steps for solving the renormalization group equation (1.4) and present the results in a form convenient for phenomenological analysis $[15,16,40]$.

The renormalization group equation (2.5) is an inhomogeneous first-order partial differential equation and after sandwiching the conformal operators between the hadronic states of interest we obtain the evolution equation for the reduced matrix elements:

$$
\begin{aligned}
\mu \frac{d}{d \mu} & \left\langle\pi(P)\left|\mathcal{O}_{j j}(\mu)\right| \Omega\right\rangle^{\mathrm{red}} \\
= & -\gamma_{j j}(\mu)\left\langle\pi(P)\left|\mathcal{O}_{j j}(\mu)\right| \Omega\right\rangle^{\mathrm{red}} \\
& \quad-\sum_{k=0}^{j-2} \gamma_{j k}(\mu)\left\langle\pi(P)\left|\mathcal{O}_{k k}(\mu)\right| \Omega\right\rangle^{\mathrm{red}} .
\end{aligned}
$$

The solution can be achieved by the ansatz

$$
\begin{aligned}
& \left\langle\pi(P)\left|\mathcal{O}_{j j}(\mu)\right| \Omega\right\rangle^{\text {red }} \\
& =\sum_{k=0}^{j}{ }^{\prime} \mathcal{B}_{j k}\left(\mu, \mu_{0}\right) \exp \left\{-\int_{\mu_{0}}^{\mu} \frac{d \mu^{\prime}}{\mu^{\prime}} \gamma_{k}\left(\mu^{\prime}\right)\right\} \\
& \quad \times\left\langle\pi(P)\left|\mathcal{O}_{k k}\left(\mu_{0}\right)\right| \Omega\right\rangle^{\mathrm{red}},
\end{aligned}
$$

with the initial condition

$$
\mathcal{B}_{j k}\left(\mu=\mu_{0}, \mu_{0}\right)=\delta_{j k} .
$$

The recursive solution of this set of differential equations, starting with the homogeneous one for $j=0$, has been written for an arbitrary scheme in a compact form (see Ref. [15]):

$$
\mathcal{B}_{j k}=\frac{\delta_{j k}}{\delta_{j k}-\mathcal{L} \gamma_{j k}^{\mathrm{ND}}}=\delta_{j k}+\mathcal{L} \gamma_{j k}^{\mathrm{ND}}+\mathcal{L}\left(\gamma^{\mathrm{ND}} \mathcal{L} \hat{\gamma}^{\mathrm{ND}}\right)_{j k}+\cdots,
$$

where $\hat{\gamma}^{\mathrm{ND}}$ represents the triangular off-diagonal matrix

$$
\gamma_{j k}^{\mathrm{ND}}=\left\{\begin{array}{cc}
\gamma_{j k} & \text { for } j>k, \\
0 & \text { otherwise }
\end{array}\right.
$$

and the operator $\mathcal{L}$ is an integral operator whose action is defined by

$$
\begin{aligned}
\mathcal{L} \gamma_{j k}^{\mathrm{ND}}= & -\int_{\mu_{0}}^{\mu} \frac{d \mu^{\prime}}{\mu^{\prime}} \gamma_{j k}^{\mathrm{ND}}\left(\mu^{\prime}\right) \\
& \times \exp \left\{-\int_{\mu^{\prime}}^{\mu} \frac{d \mu^{\prime \prime}}{\mu^{\prime \prime}}\left[\gamma_{j}\left(\mu^{\prime \prime}\right)-\gamma_{k}\left(\mu^{\prime \prime}\right)\right]\right\} .
\end{aligned}
$$

In the $\overline{\mathrm{MS}}$ or CS scheme, the anomalous dimensions (2.6) depend only implicitly on the scale $\mu$ via the running coupling $\alpha_{s}(\mu)$. For the $\beta$ function, we employ the expansion [63]

$$
\frac{\beta}{g}=\frac{\alpha_{s}(\mu)}{4 \pi} \beta_{0}+\frac{\alpha_{s}^{2}(\mu)}{(4 \pi)^{2}} \beta_{1}+\frac{\alpha_{s}^{3}(\mu)}{(4 \pi)^{3}} \beta_{2}+O\left(\alpha_{s}^{4}\right),
$$

$$
\beta_{0}=\frac{2}{3} n_{f}-11, \quad \beta_{1}=\frac{38}{3} n_{f}-102,
$$

$$
\beta_{2}=-\frac{325}{54} n_{f}^{2}+\frac{5033}{18} n_{f}-\frac{2857}{2} .
$$

Since the off-diagonal entries of the anomalous dimensions give only subleading logs, which will not be resummed, we expand the $\mathcal{B}$ matrix in powers of $\alpha_{s}$ :

$$
\begin{aligned}
\mathcal{B}_{j k}\left(\mu, \mu_{0}\right)= & \delta_{j k}+\frac{\alpha_{s}(\mu)}{2 \pi} \mathcal{B}_{j k}^{(1)}\left(\mu, \mu_{0}\right)+\frac{\alpha_{s}^{2}(\mu)}{(2 \pi)^{2}} \mathcal{B}_{j k}^{(2)}\left(\mu, \mu_{0}\right) \\
& +O\left(\alpha_{s}^{3}\right) .
\end{aligned}
$$

Performing the integrations in Eq. (2.43), we obtain the desired results for 


$$
\begin{gathered}
\mathcal{B}_{j k}^{(1)}=-R_{j k}\left(\mu, \mu_{0} \mid 1\right) \frac{\gamma_{j k}^{\mathrm{ND}(1)}}{\beta_{0}}, \\
\mathcal{B}_{j k}^{(2)}=\left[R_{j k}\left(\mu, \mu_{0} \mid 1\right)-R_{j k}\left(\mu, \mu_{0} \mid 2\right)\right]\left[\frac{\gamma_{j}^{(1)}-\gamma_{k}^{(1)}}{\beta_{0}}-\frac{\beta_{1}}{2 \beta_{0}} \frac{\gamma_{j}^{(0)}-\gamma_{k}^{(0)}}{\beta_{0}}\right] \frac{\gamma_{j k}^{\mathrm{ND}(1)}}{\beta_{0}}+R_{j k}\left(\mu, \mu_{0} \mid 2\right) \\
\times\left[\frac{\beta_{1}}{2 \beta_{0}} \frac{\gamma_{j k}^{\mathrm{ND}(1)}}{\beta_{0}}-\frac{\gamma_{j k}^{\mathrm{ND}(2)}}{\beta_{0}}\right]+\sum_{m=k+2}^{j-2} \frac{\gamma_{j m}^{\mathrm{ND}(1)}}{\beta_{0}} \frac{R_{m k}\left(\mu, \mu_{0} \mid 1\right)-R_{j m}\left(\mu, \mu_{0} \mid 2\right)}{1+\left(\gamma_{j}^{(0)}-2 \gamma_{m}^{(0)}+\gamma_{k}^{(0)}\right) / \beta_{0}} \frac{\gamma_{m k}^{\mathrm{ND}(1)}}{\beta_{0}},
\end{gathered}
$$

where

$$
R_{j k}\left(\mu, \mu_{0} \mid n\right)=\frac{\beta_{0}}{n \beta_{0}+\gamma_{j}^{(0)}-\gamma_{k}^{(0)}}\left[1-\left(\frac{\alpha_{s}\left(\mu_{0}\right)}{\alpha_{s}(\mu)}\right)^{\left(n \beta_{0}+\gamma_{j}^{(0)}-\gamma_{k}^{(0)}\right) / \beta_{0}}\right]
$$

The leading logs associated with the diagonal entries are resummed, while the subleading ones are expanded with respect to $\alpha_{s}$ :

$$
\exp \left\{-\int_{\mu_{0}}^{\mu} \frac{d \mu^{\prime}}{\mu^{\prime}} \gamma_{k}\left(\mu^{\prime}\right)\right\}=\left[\frac{\alpha_{s}(\mu)}{\alpha_{s}\left(\mu_{0}\right)}\right]^{-\gamma_{k}^{(0)} / \beta_{0}}\left[1+\frac{\alpha_{s}(\mu)}{2 \pi} \mathcal{A}_{k}^{(1)}\left(\mu, \mu_{0}\right)+\frac{\alpha_{s}^{2}(\mu)}{(2 \pi)^{2}} \mathcal{A}_{k}^{(2)}\left(\mu, \mu_{0}\right)+O\left(\alpha_{s}^{3}\right)\right]
$$

where

$$
\begin{aligned}
& \mathcal{A}_{k}^{(1)}\left(\mu, \mu_{0}\right)=\left[1-\frac{\alpha_{s}\left(\mu_{0}\right)}{\alpha_{s}(\mu)}\right]\left[\frac{\beta_{1}}{2 \beta_{0}} \frac{\gamma_{k}^{(0)}}{\beta_{0}}-\frac{\gamma_{k}^{(1)}}{\beta_{0}}\right], \\
& \mathcal{A}_{k}^{(2)}\left(\mu, \mu_{0}\right)=\frac{1}{2}\left[\mathcal{A}_{k}^{(1)}\left(\mu, \mu_{0}\right)\right]^{2}-\left[1-\frac{\alpha_{s}^{2}\left(\mu_{0}\right)}{\alpha_{s}^{2}(\mu)}\right]\left[\frac{\beta_{1}^{2}-\beta_{2} \beta_{0}}{8 \beta_{0}} \frac{\gamma_{k}^{(0)}}{\beta_{0}}-\frac{\beta_{1}}{4 \beta_{0}} \frac{\gamma_{k}^{(1)}}{\beta_{0}}+\frac{\gamma_{k}^{(2)}}{2 \beta_{0}}\right] .
\end{aligned}
$$

To the considered order, the evolution of the matrix elements then reads

$$
\begin{aligned}
\left\langle\pi(P)\left|\mathcal{O}_{j j}(\mu)\right| \Omega\right\rangle^{\mathrm{red}}= & \sum_{k=0}^{j}{ }^{\prime}\left[\frac{\alpha_{s}(\mu)}{\alpha_{s}\left(\mu_{0}\right)}\right]^{-\gamma_{k}^{(0)} / \beta_{0}}\left\{\delta_{j k}+\frac{\alpha_{s}(\mu)}{2 \pi}\left[\delta_{j k} \mathcal{A}_{k}^{(1)}+\mathcal{B}_{j k}^{(1)}\right]\left(\mu, \mu_{0}\right)\right. \\
& \left.+\frac{\alpha_{s}^{2}(\mu)}{(2 \pi)^{2}}\left[\delta_{j k} \mathcal{A}_{k}^{(2)}+\mathcal{B}_{j k}^{(1)} \mathcal{A}_{k}^{(1)}+\mathcal{B}_{j k}^{(2)}\right]\left(\mu, \mu_{0}\right)+O\left(\alpha_{s}^{3}\right)\right\}\left\langle\pi(P)\left|\mathcal{O}_{k k}\left(\mu_{0}\right)\right| \Omega\right\rangle^{\mathrm{red}} .
\end{aligned}
$$

The off-diagonal entries are known only at NLO and are given in the $\overline{\mathrm{MS}}$ scheme in Eq. (2.11). In the CS scheme, they are proportional to $\beta_{0}$, as given in Eq. (2.15), whereas in the $\overline{\mathrm{CS}}$ scheme they are equal to zero by definition. Therefore, the mixing of operators is an $\alpha_{s}^{2}$ suppressed effect:

$$
\begin{aligned}
& \mathcal{B}_{j k}^{(1)}=0, \\
& \mathcal{B}_{j k}^{(2)}=\frac{\Delta_{j k}^{(0)}}{2} \frac{\gamma_{j}^{(0)}-\gamma_{k}^{(0)}}{\beta_{0}+\gamma_{j}^{(0)}-\gamma_{k}^{(0)}}\left[\frac{\beta_{0}+\gamma_{j}^{(0)}-\gamma_{k}^{(0)}}{\beta_{0}} \ln \left(\frac{\mu^{2}}{\mu^{* 2}}\right) R_{j k}\left(\mu, \mu_{0} \mid 2\right)+\ln \left(\frac{\mu^{2}}{\mu_{0}^{2}}\right)\left(\frac{R_{j k}\left(\mu, \mu_{0} \mid 2\right)}{1-\alpha_{s}\left(\mu_{0}\right) / \alpha_{s}(\mu)}-1\right)\right] \\
& -\frac{1}{2} R_{j k}\left(\mu, \mu_{0} \mid 2\right) \Delta_{j k}^{\overline{\mathrm{CS}}(1)},
\end{aligned}
$$

where $\Delta_{j k}^{(0)}$ is defined in Eq. (2.16). Here we have taken into account the explicit $\mu$ dependence in the anomalous dimensions, induced by the transformation (2.29). The addendum $\Delta_{j k}^{\overline{\mathrm{CS}}(1)}=2 \gamma_{j k}^{\overline{\mathrm{CS}}(2)} / \beta_{0}$, which is presently unknown, has to be evaluated at $\mu=\mu^{*}$. Note that the $\alpha_{s}$ power counting remains correct as long as the scales $\mu^{*}, \mu$, and $\mu_{0}$ are of the order of $Q \gg \Lambda_{\mathrm{QCD}}$. The auxiliary scale can now be set, e.g., to $\mu^{*}=\mu$.

Let us remark that the evolution of the distribution amplitude can be formally obtained by resummation of the conformal partial waves given in Eq. (2.1). Taking into account the evolution of the reduced matrix element in Eq. (2.52), one 
finds the eigenfunctions of the evolution equation, expanded with respect to the Gegenbauer polynomials [15].

\section{HARD-SCATTERING AMPLITUDE TO NNLO ACCURACY}

In the preceding section we outlined the structure of the conformal predictions in the conformal momentum space and in this one we analyze the structure of the NNLO results in the momentum fraction representation. In Sec. III A we derive a convolution representation of the NNLO term proportional to $\beta_{0}$ and also give the general structure of the hard-scattering amplitude in the $\overline{\mathrm{MS}}$ scheme up to the NNLO order. Furthermore, in Sec. III B we consider the phenomenologically important case of the asymmetry parameter $|\omega|$ equal to 1 and in Sec. III C we then present the NNLO result for the conformal moments in the $\mathrm{CS}$ and $\overline{\mathrm{CS}}$ schemes. In Sec. III D we analogously present the results at small and the intermediate values of $|\omega|$.

\section{A. $\boldsymbol{\beta}_{0}$-proportional NNLO terms and the general structure of the NNLO results in the $\overline{M S}$ scheme}

First, we consider the term proportional to $n_{f}$, i.e., $\beta_{0}$, appearing in the NNLO calculation of the two-photon hardscattering amplitude (1.2). For the case of general Bjorken kinematics, the result has been given in Ref. [9] and is easily restricted to the kinematics of a particular process, i.e., in our case, to the kinematics of the photon-to-pion transition form factor (see Appendix B for the definitions of generalized Bjorken kinematics). In the special case $|\omega|=1$ (one photon on shell), these results coincide with the results from Ref. [10].

The authors of Ref. [9] presented the regularized result in terms of hypergeometric functions ${ }_{2} F_{1}$. It is instructive to rewrite it as a convolution of the amplitude

$$
T^{(0)}(\omega, x \mid \epsilon)=\frac{1}{[1-\omega(2 x-1)]^{1+\epsilon}},
$$

with the kernels

$$
\begin{aligned}
v^{a}(x, y \mid \epsilon)= & \theta(y-x)\left(\frac{x}{y}\right)^{1+\epsilon}+\left\{\begin{array}{l}
x \rightarrow \bar{x} \\
y \rightarrow \bar{y}
\end{array}\right\}, \\
v^{b}(x, y \mid \epsilon)= & \theta(y-x)\left(\frac{x}{y}\right)^{1+\epsilon} \frac{1}{y-x}+\left\{\begin{array}{c}
x \rightarrow \bar{x} \\
y \rightarrow \bar{y}
\end{array}\right\}, \\
g(x, y \mid \epsilon, \sigma)= & \theta(y-x) \frac{1}{y}\left(1-\frac{x}{y}\right)^{-1+\epsilon+\sigma} \\
& \times \mathrm{B}\left(\frac{x}{y}, 1+\sigma,-\epsilon-\sigma\right)+\left\{\begin{array}{l}
x \rightarrow \bar{x} \\
y \rightarrow \bar{y}
\end{array}\right\},
\end{aligned}
$$

where $\mathrm{B}(x, a, b)=\int_{0}^{x} d y y^{a-1}(1-y)^{b-1}$ is the incomplete Beta function. Here $\sigma$ and $\epsilon$ are the dimensional regularization parameters $(n=4-2 \sigma[\epsilon])$ associated with the quark- bubble insertion and the overall loop, respectively. The kernels $\left[v^{a}(x, y \mid \epsilon)\right]_{+}$and $\left[v^{b}(x, y \mid \epsilon)\right]_{+}$are diagonal with respect to the Gegenbauer polynomials $C_{j}^{3 / 2+\epsilon}(2 x-1)$ and they are regularized with the usual $[\cdots]_{+}$prescription:

$$
[v(x, y)]_{+}=v(x, y)-\delta(x-y) \int_{0}^{1} d z v(z, y) .
$$

Their eigenvalues are

$$
\begin{aligned}
& v_{j}^{a}=\frac{1+\epsilon}{(1+j+\epsilon)(2+j+\epsilon)}-\frac{1}{2+\epsilon}, \\
& v_{j}^{b}=2 \psi(2+\epsilon)-2 \psi(2+\epsilon+j) .
\end{aligned}
$$

The $g$ kernel is not diagonal with respect to the Gegenbauer polynomials and is responsible for the apparent breaking of conformal symmetry in the $\overline{\mathrm{MS}}$ scheme. Its expansion $g(x, y \mid \epsilon, \sigma)=g(x, y)+g^{\prime}(x, y) \epsilon+\dot{g}(x, y) \sigma+O\left(\epsilon^{2}, \sigma^{2}, \epsilon \sigma\right)$

reads

$$
\begin{gathered}
g(x, y)=-\frac{\theta(y-x)}{y-x} \ln \left(1-\frac{x}{y}\right)+\left\{\begin{array}{c}
x \rightarrow \bar{x} \\
y \rightarrow \bar{y}
\end{array}\right\}, \\
g^{\prime}(x, y)=-\frac{\theta(y-x)}{y-x} \frac{1}{2} \ln ^{2}\left(1-\frac{x}{y}\right)+\left\{\begin{array}{c}
x \rightarrow \bar{x} \\
y \rightarrow \bar{y}
\end{array}\right\}, \\
\dot{g}(x, y)=\frac{\theta(y-x)}{y-x}\left[\operatorname{Li}_{2}\left(1-\frac{x}{y}\right)-\operatorname{Li}_{2}(1)\right] \\
+\left\{\begin{array}{c}
x \rightarrow \bar{x} \\
y \rightarrow \bar{y}
\end{array}\right\}+g^{\prime}(x, y) .
\end{gathered}
$$

There is a similar expansion of the $v^{i}$ kernels $(i=a, b)$

$$
v^{i}(x, y \mid \epsilon)=v^{i}(x, y)+\dot{v}^{i}(x, y) \epsilon+\frac{1}{2} \ddot{v} i(x, y) \epsilon^{2}+O\left(\epsilon^{3}\right),
$$

where

$$
\begin{aligned}
& v^{i}(x, y)=\theta(y-x) f^{i}(x, y)+\left\{\begin{array}{l}
x \rightarrow \bar{x} \\
y \rightarrow \bar{y}
\end{array}\right\}, \\
& \dot{v}^{i}(x, y)=\theta(y-x) f^{i}(x, y) \ln \left(\frac{x}{y}\right)+\left\{\begin{array}{l}
x \rightarrow \bar{x} \\
y \rightarrow \bar{y}
\end{array}\right\}, \\
& \ddot{v}^{i}(x, y)=\theta(y-x) f^{i}(x, y) \ln ^{2}\left(\frac{x}{y}\right)+\left\{\begin{array}{l}
x \rightarrow \bar{x} \\
y \rightarrow \bar{y}
\end{array}\right\},
\end{aligned}
$$

and the functions $f^{i}$ can be read off from Eqs. (3.2), (3.3).

The LO kernel of Eq. (1.5) is expressed in terms of the $v^{i}$ kernels introduced above:

$$
V^{(0)}(x, y)=C_{F}[v(x, y)]_{+}, \quad v(x, y)=v^{a}(x, y)+v^{b}(x, y) .
$$

For the NLO kernel we use the color decomposition 


$$
\begin{aligned}
V^{(1)}(x, y)= & C_{F}\left[C_{F} v_{F}(x, y)-\frac{\beta_{0}}{2} v_{\beta}(x, y)\right. \\
& \left.-\left(C_{F}-\frac{C_{A}}{2}\right) v_{G}(x, y)\right]_{+} .
\end{aligned}
$$

In the following we particularly need the $\beta_{0}$-proportional kernel

$$
v_{\beta}(x, y)=\dot{v}(x, y)+\frac{5}{3} v(x, y)+v^{a}(x, y) .
$$

The unrenormalized NLO and NNLO corrections to the hard-scattering amplitude are of the form

$$
\hat{T}^{(1)}(\omega, x)=C_{F} \hat{T}_{F}^{(1)}(\omega, x),
$$

$$
\begin{aligned}
\hat{T}^{(2)}(\omega, x)= & C_{F}\left[C_{F} \hat{T}_{F}^{(2)}(\omega, x)-\frac{\beta_{0}}{2} \hat{T}_{\beta}^{(2)}(\omega, x)\right. \\
& \left.-\left(C_{F}-\frac{C_{A}}{2}\right) \hat{T}_{G}^{(2)}(\omega, x)\right] .
\end{aligned}
$$

Employing the integral representation of the hypergeometric functions, one can express the regularized results of Ref. [9]

$$
\begin{gathered}
\hat{T}_{F}^{(1)}(\omega, x)=\mathcal{M}(\omega, x \mid \epsilon, 0), \\
\hat{T}_{\beta}^{(2)}(\omega, x)=-3 \frac{\Gamma(\epsilon) \Gamma(2-\epsilon)^{2}}{\Gamma(4-2 \epsilon)} \mathcal{M}(\omega, x \mid \epsilon, \epsilon)
\end{gathered}
$$

in terms of convolutions

$$
\begin{aligned}
\mathcal{M}(\omega, x \mid \epsilon, \sigma)= & \frac{\Gamma(\epsilon+\sigma) \Gamma(2-\epsilon) \Gamma(1-\epsilon-\sigma)}{\Gamma(3-2 \epsilon-\sigma) \Gamma(1+\sigma)}\left(\frac{4 \pi \mu^{2}}{Q^{2}}\right)^{\epsilon+\sigma} \int_{0}^{1} d y T^{(0)}(\omega, y \mid \epsilon+\sigma)\left\{-\frac{(1-\epsilon)(1-4 \epsilon-3 \sigma)}{1+\sigma} \delta(x-y)\right. \\
& +\frac{(1-\epsilon)[2-2 \epsilon(1-\epsilon)+\sigma(1-\sigma)]}{1+\sigma} v^{a}(y, x \mid \sigma)+\frac{(1-\epsilon)[2-\epsilon+2 \epsilon(\epsilon+\sigma)]-\epsilon \sigma(\epsilon+\sigma)}{1-\epsilon} \\
& \left.\times\left(\left[v^{b}(y, x \mid \sigma)\right]_{+}-(\epsilon+\sigma)[g(y, x \mid \epsilon, \sigma)]_{+}\right)\right\} .
\end{aligned}
$$

The results given above contain UV and collinear singularities, which are removed by renormalization (introduces the scale $\mu_{r}$ ) and factorization (at the scale $\mu_{f}$ ) of collinear singularities. The renormalization procedure in the $\overline{\mathrm{MS}}$ scheme (for details see Ref. [10]) induces the following general structure of NLO and NNLO corrections present in the expansion (1.2):

$$
\begin{aligned}
T^{(1)}\left(\omega, x, Q / \mu_{f}\right)= & C_{F} T_{F}^{(1)}(\omega, x)+\ln \left(\frac{Q^{2}}{\mu_{f}^{2}}\right)\left[T^{(0)} \otimes V^{(0)}\right](\omega, x), \\
T^{(2)}\left(\omega, x, Q / \mu_{f}, Q / \mu_{r}\right)= & C_{F}\left[C_{F} T_{F}^{(2)}-\frac{\beta_{0}}{2} T_{\beta}^{(2)}-\left(C_{F}-\frac{C_{A}}{2}\right) T_{G}^{(2)}\right](\omega, x) \\
& +\ln \left(\frac{Q^{2}}{\mu_{f}^{2}}\right)\left\{T^{(0)} \otimes V^{(1)}+\left[C_{F} T_{F}^{(1)}+\frac{1}{2} \ln \left(\frac{Q^{2}}{\mu_{f}^{2}}\right) T^{(0)} \otimes V^{(0)}\right] \otimes V^{(0)}\right\}(\omega, x) \\
& +\frac{\beta_{0}}{2} \ln \left(\frac{Q^{2}}{\mu_{r}^{2}}\right) T^{(1)}\left(\omega, x, Q / \mu_{f}\right)-\frac{\beta_{0}}{4} \ln ^{2}\left(\frac{Q^{2}}{\mu_{f}^{2}}\right)\left[T^{(0)} \otimes V^{(0)}\right](\omega, x),
\end{aligned}
$$

where

$$
\begin{aligned}
& T_{F}^{(1)}(\omega, x)=T^{(0)}(\omega, y) \otimes\left\{\mathcal{T}_{F}^{(1)}(y, x)+\mathrm{LN}(\omega, y)[v(y, x)]_{+}\right\}, \\
& T_{F}^{(2)}(\omega, x)=T^{(0)}(\omega, y) \otimes\left\{\mathcal{T}_{F}^{(2)}(y, x)+\mathrm{LN}(\omega, y)\left(\left[v_{F}(y, x)\right]_{+}+\mathcal{T}_{F}^{(1)} \otimes[v]_{+}(y, x)\right)+\frac{1}{2} \mathrm{LN}^{2}(\omega, y)[v]_{+} \otimes[v]_{+}(y, x)\right\}, \\
& T_{\beta}^{(2)}(\omega, x)=T^{(0)}(\omega, y) \otimes\left\{\mathcal{T}_{\beta}^{(2)}(y, x)+\mathrm{LN}(\omega, y)\left(\left[v_{\beta}\right]_{+}-\mathcal{T}_{F}^{(1)}\right)(y, x)-\frac{1}{2} \mathrm{LN}^{2}(\omega, y)[v(y, x)]_{+}\right\}, \\
& T_{G}^{(2)}(\omega, x)=T^{(0)}(\omega, y) \otimes\left\{\mathcal{T}_{G}^{(2)}(y, x)+\mathrm{LN}(\omega, y)\left[v_{G}(y, x)\right]_{+}\right\},
\end{aligned}
$$

while $\mathrm{LN}(\omega, x)=\ln [1+\omega-2 x \omega]$ and $T^{(0)}(\omega, x)$ is given by Eq. (1.3). For a detailed discussion of the appearance of the LN terms, see Appendix A. 
The explicit calculation provides us with the kernels

$$
\begin{aligned}
& \mathcal{T}_{F}^{(1)}(x, y)=\left[-\frac{3}{2} v^{b}+g\right]_{+}(x, y)-\frac{3}{2} \delta(x-y), \\
& \mathcal{T}_{\beta}^{(2)}(x, y)=\left[\frac{29}{12} v^{a}+\dot{v}^{a}-\frac{209}{36} v-\frac{7}{3} \dot{v}-\frac{1}{4} \ddot{v}+\frac{19}{6} g+\dot{g}\right]_{+}(x, y)-3 \delta(x-y) .
\end{aligned}
$$

As we see, these $\mathcal{T}$ kernels are built up of $v$ and $g$ kernels also appearing in the evolution kernel. While the $g$ and $\dot{v}$ kernels appear at NLO, the $\dot{g}$ and $\ddot{v}$ ones show up for the first time at NNLO. For the missing two entries, namely, $\mathcal{T}_{F}^{(2)}$ and $\mathcal{T}_{G}^{(2)}$, we expect a similar structure, but with additional and unknown building blocks that are related to the $g$ kernel.

Making use of the fact that both the photon-to-pion transition form factor and the forward Compton scattering belong to the class of light-cone dominated two-photon processes, which can be described by a general scattering amplitude, we have performed a consistency check between the previously presented results for the hard-scattering amplitude of the photon-to-pion transition form factor (known up to $\beta_{0}$-proportional NNLO terms) and the corresponding results for the nonsinglet coefficient function of the DIS polarized structure function $g_{1}$ [43]. The procedure is presented in detail in Appendix B.

A few comments regarding the $\mathrm{LN}(\omega, x)$ terms are in order. In NLO, we observe that the $\operatorname{LN}(\omega, x)$ term matches the In term indicated in Eq. (3.16), i.e., we can absorb it in Eq. (3.18) by an appropriate choice of the scale:

$$
\mu_{i}^{2} \rightarrow \tilde{\mu}_{i}^{2}=\mu_{i}^{2}(1+\omega-2 x \omega)^{-1} \text { with } i=\{f, r\} .
$$

The explicit NNLO result for the $\beta$-proportional terms satisfies the same rule for the scale redefinition, which indicates a general property of the hard-scattering amplitude evaluated in the $\overline{\mathrm{MS}}$ scheme. This is shown in Appendix A. The terms proportional to $\mathrm{LN}(\omega, x)$ are vanishing in the limit $|\omega| \rightarrow 0$ and for $|\omega| \rightarrow 1$ provide a logarithmic enhancement in the end-point region. However, a resummation of such terms through an appropriate scale setting is misleading, since other logarithmically enhanced terms also appear. For instance, at NLO we have

$$
\begin{aligned}
\frac{\ln (1-y)}{1-y} \otimes[v(y, x)]_{+}= & \frac{\ln ^{2}(1-x)+2 \ln (1-x)}{1-x} \\
& +O(\ln (1-x)),
\end{aligned}
$$

while the contribution of $\mathcal{T}_{F}^{(1)}(x, y)$ is [see Eq. (3.22)]

$$
\begin{aligned}
\frac{1}{1-y} \otimes \mathcal{T}_{F}^{(1)}(y, x)= & -\frac{\ln ^{2}(1-x)+3 \ln (1-x)+9}{2(1-x)} \\
& +O(\ln (1-x))
\end{aligned}
$$

so that only a partial cancellation of the $\ln ^{2}(1-x) /(1-x)$ term appears.

\section{B. Limit $|\omega| \rightarrow 1$ and corresponding conformal moments in the $\overline{M S}$ scheme}

Of special interest is the limit $|\omega| \rightarrow 1$, since different (pseudoscalar) meson-to-photon transition form factors are measured in this kinematical region. We can trivially perform this limit in Eqs. (1.3), (3.18), and (3.20) and after convolution we present the result in the form of Ref. [10]:

$T^{(0)}(x)=\frac{1}{2(1-x)}$,

$T_{F}^{(1)}(x)=\frac{1}{2(1-x)}\left[-\frac{9}{2}-\frac{1-x}{2 x} \ln (1-x)+\frac{1}{2} \ln ^{2}(1-x)\right]$,

$$
\begin{aligned}
T_{\beta}^{(2)}(x)= & \frac{1}{2(1-x)}\left[-\frac{457}{48}-\left(\frac{47}{36}-\frac{1}{4 x}\right) \ln (1-x)\right. \\
& +\left(\frac{13}{12}-\frac{1}{4 x}\right) \ln ^{2}(1-x)-\frac{1}{6} \ln ^{3}(1-x)-\frac{7}{3} \operatorname{Li}_{2}(x) \\
& \left.+\frac{1}{2} \operatorname{Li}_{3}(x)-\mathrm{S}_{12}(x)\right]
\end{aligned}
$$

where the polylogarithms are defined by

$$
\begin{aligned}
\operatorname{Li}_{n}(x)= & \int_{0}^{x} d y \frac{\operatorname{Li}_{n-1}(y)}{y} \text { with } \operatorname{Li}_{1}(x)=-\ln (1-x), \\
\mathrm{S}_{12}(x)= & \frac{1}{2} \int_{0}^{x} d y \frac{\ln ^{2}(1-y)}{y} \\
= & \frac{1}{2} \ln ^{2}(1-x) \ln (x)+\ln (1-x) \mathrm{Li}_{2}(1-x) \\
& -\operatorname{Li}_{3}(1-x)+\zeta(3) .
\end{aligned}
$$

Here we introduce for the special case $|\omega|=1$ a more convenient notation in which the $\ln (2)$ terms arising from the 
LN $(\omega=1, x)$ functions present in Eqs. (3.18)-(3.20) are absorbed in $\ln \left(2 Q^{2} / \mu_{f,(r)}^{2}\right)=\ln \left(-q_{1}^{2} / \mu_{f,(r)}^{2}\right)$. We do not list here the terms proportional to these logs but refer to Ref. [10] for their explicit expressions.
The conformal moments of the individual terms we encounter in Eqs. (3.27)-(3.29) are given in Appendix C. Here we list the conformal moments of $T^{(0)}(x), T_{F}^{(1)}(x)$, and $T_{\beta}^{(2)}(x)$ calculated in the $\overline{\mathrm{MS}}$ scheme:

$$
\begin{gathered}
T_{j}^{(0)}=\frac{2 j+3}{(j+1)(j+2)}, \\
T_{F, j}^{(1)}=\frac{2 j+3}{(j+1)(j+2)}\left[-\frac{9}{2}+\frac{3-4 S_{1}(j+1)}{2(j+1)(j+2)}+\frac{1}{(j+1)^{2}(j+2)^{2}}+2 S_{1}^{2}(j+1)\right], \\
T_{\beta, j}^{(2)}=\frac{2 j+3}{(1+j)(2+j)}\left[-\frac{457}{48}-\frac{1}{2} \zeta(3)-\frac{7}{3} \zeta(2)+\frac{1}{(j+1)(j+2)}\left(\frac{115}{36}-\frac{(-1)^{j}}{2} \zeta(2)-\frac{1}{3} S_{1}(j+1)-2 S_{1}^{2}(j+1)\right.\right. \\
\left.\left.-(-1)^{j} S_{-2}(j+1)\right)-\frac{1-8 S_{1}(j+1)}{2(j+1)^{2}(j+2)^{2}}-\frac{5}{2(j+1)^{3}(j+2)^{3}}+\frac{19}{9} S_{1}(j+1)+\frac{10}{3} S_{1}^{2}(j+1)+\frac{4}{3} S_{1}^{3}(j+1)+\frac{2}{3} S_{3}(j+1)\right] .
\end{gathered}
$$

After inspection of Eqs. (3.16) and (3.17) one notes that the conformal moments of the terms proportional to $\ln \left(2 Q^{2} / \mu_{f,(r)}^{2}\right)$ can be conveniently expressed using the conformal moments of the kernels $v$ and $v_{\beta}$. For the definition of conformal moments of the kernels, we refer to Appendix C, Eq. (C7). The conformal moments of the diagonal kernel $[v(x, y)]_{+}$, given in Eq. (3.9), are denoted by $v_{j} \equiv v_{j j}$ :

$$
v_{j}=-2 S_{1}(j+1)+\frac{3}{2}+\frac{1}{(1+j)(2+j)} .
$$

The conformal moments of $T^{(0)} \otimes[v]_{+}$, determined using Eq. (C10), are given by $T_{j}^{0} v_{j}$. On the other hand, the kernel $\left[v_{\beta}\right]_{+}$in Eq. (3.11) is nondiagonal, and as shown in Eq. (C8) both the diagonal $v_{\beta, j} \equiv v_{\beta, j j}$ as well as the nondiagonal $v_{\beta, k j}(k>j$ and $k-j$ even) conformal moments contribute to the conformal moments of $T^{(0)} \otimes\left[v_{\beta}\right]_{+}$, i.e., one obtains $\Sigma_{k>j} T_{k}^{0} v_{\beta, k j}$. After performing the convolution and making use of the results for the conformal moments summarized in Appendix $\mathrm{C}$, one can express the conformal moment of $T^{(0)} \otimes\left[v_{\beta}\right]_{+}$in the form $T_{j}^{0} v_{\beta, j}^{\Sigma}$, where we introduce the "effective" conformal moment of the $v_{\beta}$ kernel amounting to

$$
v_{\beta, j}^{\Sigma}=\frac{5}{3} v_{j}+\zeta(2)-\frac{9}{4}-\frac{1}{(1+j)^{2}(2+j)^{2}} .
$$

Finally, we summarize our $\overline{\mathrm{MS}}$ results. The LO contribution is given in Eq. (3.31), the NLO contribution takes the form

$$
T_{j}^{(1)}=T_{F, j}^{(1)}+\ln \left(\frac{2 Q^{2}}{\mu_{f}^{2}}\right) \frac{2 j+3}{(j+1)(j+2)} v_{j},
$$

while the $\left(-\beta_{0} / 2\right)$-proportional NNLO term is given by

$$
\begin{aligned}
& T_{\beta, j}^{(2)}+\ln \left(\frac{2 Q^{2}}{\mu_{f}^{2}}\right) \frac{2 j+3}{(j+1)(j+2)}\left[v_{\beta, j}^{\Sigma}+\frac{1}{2} \ln \left(\frac{2 Q^{2}}{\mu_{f}^{2}}\right) v_{j}\right] \\
& -\ln \left(\frac{2 Q^{2}}{\mu_{r}^{2}}\right) T_{j}^{(1)} .
\end{aligned}
$$

Note that, owing to the fact that $v_{\beta}$ is nondiagonal, even the lowest partial wave, i.e., $j=0$, of the NNLO correction depends on the factorization scale as well as the renormalization one.

\section{NNLO result in the CS and $\overline{C S}$ schemes in the limit $|\omega| \rightarrow 1$}

Let us now turn to the conformal schemes CS and $\overline{\mathrm{CS}}$. We make a distinction between the renormalization and factorization scales. Consequently, the argument of the coupling in the Wilson coefficients depends on $\mu_{r}$ and, as discussed in Sec. II C, we require that the matrix elements of conformal operators depend only on the scale $\mu_{f}$. We use the COPE, where for $|\omega|=1$ the Wilson coefficients (2.18) simplify to 


$$
\begin{aligned}
\bar{C}_{j}= & c_{j}\left(\alpha_{s}\left(\mu_{r}\right)\right) \\
& \times\left(\frac{\mu_{f}^{2}}{2 Q^{2}}\right)^{\gamma_{j} / 2} \frac{\Gamma(j+1) \Gamma(j+2) \Gamma\left(2 j+4+\gamma_{j}\right)}{\Gamma\left(j+2+\gamma_{j} / 2\right) \Gamma\left(j+3+\gamma_{j} / 2\right) \Gamma(2 j+3)} .
\end{aligned}
$$

The anomalous dimensions are given by

$$
\begin{aligned}
\gamma_{j}= & \frac{\alpha_{s}\left(\mu_{r}\right)}{2 \pi} \gamma_{j}^{(0)}+\frac{\alpha_{s}^{2}\left(\mu_{r}\right)}{(2 \pi)^{2}} \gamma_{j}^{(1)}+O\left(\alpha_{s}^{3}\right) \\
= & -2\left\{\frac{\alpha_{s}\left(\mu_{r}\right)}{2 \pi} C_{F} v_{j}+\frac{\alpha_{s}\left(\mu_{r}\right)}{(2 \pi)^{2}} C_{F}\left[C_{F} v_{F, j}-\frac{\beta_{0}}{2} v_{\beta, j}\right.\right. \\
& \left.\left.-\left(C_{F}-\frac{1}{2} C_{A}\right) v_{G, j}\right]+O\left(\alpha_{s}^{3}\right)\right\},
\end{aligned}
$$

where $v_{j}, v_{F, j}, v_{G, j}$, and $v_{\beta, j}$ are the diagonal conformal moments of the evolution kernels $[v(x, y)]_{+},\left[v_{F}(x, y)\right]_{+}$, $\left[v_{G}(x, y)\right]_{+}$, and $\left[v_{\beta}(x, y)\right]_{+}$, respectively, and they coincide with the moments of the DGLAP kernels. The LO moments are given by Eq. (3.34), while other entries can be found in Refs. [54-57]. Analogously, we decompose the normalization factor

$$
\begin{aligned}
c_{j}= & 1+\frac{\alpha_{s}}{2 \pi} C_{F} c_{j}^{(1)}+\left(\frac{\alpha_{s}}{2 \pi}\right)^{2} C_{F}\left[C_{F} c_{F, j}^{(2)}-\frac{\beta_{0}}{2} c_{\beta, j}^{(2)}\right. \\
& \left.-\left(C_{F}-\frac{1}{2} C_{A}\right) c_{G, j}^{(2)}\right]+O\left(\alpha_{s}^{3}\right) .
\end{aligned}
$$

Its NLO contribution reads

$$
\begin{aligned}
c_{j}^{(1)}= & S_{1}^{2}(1+j)+\frac{3}{2} S_{1}(j+2)-\frac{9}{2}+\frac{3-2 S_{1}(j)}{2(j+1)(j+2)} \\
& -S_{2}(j+1),
\end{aligned}
$$

while the NNLO contributions can be determined from the Mellin moments of the coefficient functions calculated from the $\alpha_{s}^{2}$ corrections to the polarized structure function $g_{1}$ [43]. A consistency check of the $\beta_{0}$-proportional part of these results is given in Appendix B.

As discussed in Sec. II C, the Wilson coefficients in the CS scheme are obtained in a straightforward manner by means of Eq. (2.38), where $C_{\beta, j}^{\overline{\mathrm{MS}}(2)}\left(\alpha_{s}\left(\mu_{r}\right), Q / \mu_{f}, Q / \mu_{r}\right)$ is given by the expression (3.37). Taking into account the proper normalization, i.e., identifying $T^{(i)}$ with $C^{(i)}$ by Eq. (2.17), the expansion of $\bar{C}_{j}$, defined by Eq. (3.38), leads to the complete NNLO result for the hard-scattering amplitude:

$$
\begin{aligned}
T_{j}^{\mathrm{CS}}\left(Q, \mu_{f}\right)= & \frac{\sqrt{2}}{3 Q^{2}}\left[T_{j}^{(0)}+\frac{\alpha_{s}\left(\mu_{r}\right)}{2 \pi} T_{j}^{\mathrm{CS}(1)}\left(Q / \mu_{f}\right)\right. \\
& \left.+\frac{\alpha_{s}^{2}\left(\mu_{r}\right)}{(2 \pi)^{2}} T_{j}^{\mathrm{CS}(2)}\left(Q / \mu_{f}, Q / \mu_{r}\right)+O\left(\alpha_{s}^{3}\right)\right],
\end{aligned}
$$

where

$$
\begin{aligned}
T_{j}^{\mathrm{CS}(1)}= & C_{F}\left[T_{F, j}^{\mathrm{CS}(1)}+\frac{2 j+3}{(j+1)(j+2)} \ln \left(\frac{2 Q^{2}}{\mu_{f}^{2}}\right) v_{j}\right], \\
T_{j}^{\mathrm{CS}(2)}= & C_{F}\left\{C_{F} T_{F, j}^{\mathrm{CS}(2)}-\frac{\beta_{0}}{2} T_{\beta, j}^{(2)}-\left(C_{F}-\frac{1}{2} C_{A}\right) T_{G, j}^{\mathrm{CS}(2)}+\ln \left(\frac{2 Q^{2}}{\mu_{f}^{2}}\right) \frac{2 j+3}{(j+1)(j+2)}\right. \\
& \times\left\{C_{F}\left[v_{F, j}+c_{j}^{(1)} v_{j}+v_{j}^{2}\left[S_{1}(j+1)+S_{1}(j+2)-2 S_{1}(2 j+3)\right]+\frac{v_{j}^{2}}{2} \ln \left(\frac{2 Q^{2}}{\mu_{f}^{2}}\right)\right]-\frac{\beta_{0}}{2} v_{\beta, j}^{\mathrm{CS}, \Sigma}-\left(C_{F}-\frac{1}{2} C_{A}\right) v_{G, j}\right\} \\
& \left.+\frac{\beta_{0}}{2} \ln \left(\frac{2 Q^{2}}{\mu_{r}^{2}}\right) T_{j}^{\mathrm{CS}(1)}\left(Q / \mu_{f}\right)-\frac{\beta_{0}}{4} \ln ^{2}\left(\frac{2 Q^{2}}{\mu_{f}^{2}}\right) \frac{2 j+3}{(j+1)(j+2)} v_{j}\right\},
\end{aligned}
$$

and

$$
\begin{aligned}
T_{F, j}^{\mathrm{CS}(1)}= & \frac{2 j+3}{(j+1)(j+2)}\left\{c_{j}^{(1)}+v_{j}\left[S_{1}(j+2)+S_{1}(j+1)-2 S_{1}(2 j+3)\right]\right\} \\
T_{F, j}^{\mathrm{CS}(2)}= & \frac{2 j+3}{(j+1)(j+2)}\left\{c_{F, j}^{(2)}+\left(c_{j}^{(1)} v_{j}+v_{F, j}\right)\left[S_{1}(j+1)+S_{1}(j+2)-2 S_{1}(2 j+3)\right]+\frac{v_{j}^{2}}{2}\left[\left(S_{1}(j+1)+S_{1}(j+2)\right.\right.\right. \\
& \left.\left.\left.-2 S_{1}(2 j+3)\right)^{2}+S_{2}(j+1)+S_{2}(j+2)-4 S_{2}(2 j+3)+2 \zeta(2)\right]\right\},
\end{aligned}
$$


$T_{G, j}^{\mathrm{CS}(2)}=\frac{2 j+3}{(j+1)(j+2)}\left\{c_{G, j}^{(2)}+v_{G, j}\left[S_{1}(j+1)+S_{1}(j+2)-2 S_{1}(2 j+3)\right]\right\}$,

while $T_{\beta, j}^{(2)}$ corresponds to the $\overline{\mathrm{MS}}$ result given by Eq. (3.33). Note that, in accordance with the renormalization group invariance, the off-diagonal part of the anomalous dimension matrix proportional to $\beta_{0}$ has been changed and, consequently, also the conformal moments

$$
v_{\beta, j}^{\Sigma} \Rightarrow v_{\beta, j}^{\mathrm{CS}, \Sigma}=v_{\beta, j}^{\Sigma}+\frac{(j+1)(j+2)}{2 j+3}\left(T_{F, j}^{\mathrm{CS}(1)}-T_{F, j}^{(1)}\right) .
$$

Finally, we present the result for the $\overline{\mathrm{CS}}$ scheme, in which the conformal covariance of the partial wave decomposition is preserved. The modification concerns only the terms proportional to $\beta_{0}$ in which the off-diagonal entries in Eq. (3.44) are removed by making the following replacements:

$$
\begin{aligned}
v_{\beta, j}^{\mathrm{CS}, \Sigma} \Rightarrow v_{\beta, j}, & \\
T_{\beta, j}^{(2)} \Rightarrow T_{\beta, j}^{\overline{\mathrm{CS}}(2)}= & \frac{2 j+3}{(j+1)(j+2)}\left\{c_{\beta, j}^{(2)}+v_{\beta, j}\left[S_{1}(j+1)\right.\right. \\
& \left.\left.+S_{1}(j+2)-2 S_{1}(2 j+3)\right]\right\} \\
& +\ln (2)\left[T_{F, j}^{\mathrm{CS}(1)}+T_{j}^{(0)} \frac{1}{2} \ln (2) v_{j}\right]
\end{aligned}
$$

The $\ln (2)$ terms appear here artificially from the absorption of such terms into the factorization and renormalization logs, i.e., $\ln \left(Q^{2} / \mu_{i}^{2}\right) \rightarrow \ln \left(2 Q^{2} / \mu_{i}^{2}\right)-\ln (2)$ [see Eq. (2.35)]. All other expressions in Eqs. (3.43)-(3.47) remain unchanged, e.g.,

$$
T_{j}^{\overline{\mathrm{CS}}}(1)=T_{j}^{\mathrm{CS}(1)}, \quad T_{F, j}^{\overline{\mathrm{CS}}(2)}=T_{F, j}^{\mathrm{CS}(2)}, \quad T_{G, j}^{\overline{\mathrm{CS}}(2)}=T_{G, j}^{\mathrm{CS}(2)} .
$$

\section{NNLO predictions for small and intermediate values of $|\omega|$}

Based on numerical observations on the small and intermediate $|\omega|$ behavior of the transition form factor predicted by perturbation theory, interesting phenomenological aspects have been pointed out in Ref. [30]. Unfortunately, for these configurations both photons are virtual [cf. Eq. (1.1)] and thus the statistics is rather low. Therefore, no measurements has been done yet, although they could be possible at the existing $e^{+} e^{-}$machines of the Babar, Belle, and CLEO experiments. In the following we want to add some comments on the pion transition form factor in the small and intermediate $|\omega|$ regions and to give predictions at NNLO.

From the representation (2.34) it follows that the $j$ th conformal moments for $|\omega|<1$ are suppressed by $\omega^{j}$. In addition, the hypergeometric functions appearing in the Wilson coefficients are sharply peaked at $|\omega|=1$ owing to a logarithmic enhancement caused by the $\ln [(1-\omega) /(1+\omega)]$ term. For fixed $|\omega|<1$, one finds for growing $j$ an increasing suppression of the hypergeometrical functions, in addition to the powerlike suppression due to $\omega^{j}$. To study this behavior in more detail, we employ the integral representation for the hypergeometrical functions:

$$
\begin{aligned}
& \frac{2^{j+1+\gamma_{j} / 2}}{(1+\omega)^{j+1+\gamma_{j} / 2}}{ }_{2} F_{1}\left(\begin{array}{c|c}
j+1+\frac{1}{2} \gamma_{j}, j+2+\frac{1}{2} \gamma_{j} & \frac{2 \omega}{1+\omega} \\
2\left(j+2+\frac{1}{2} \gamma_{j}\right) &
\end{array}\right) \\
& =\frac{\Gamma\left(4+2 j+\gamma_{j}\right)}{\Gamma\left(2+j+\gamma_{j} / 2\right)^{2}} \int_{0}^{\infty} d s s e^{-s^{2} / 2} e^{\left[\left(1+j+\gamma_{j} / 2\right) / 2\right]\left\{-s^{2}+\ln \left(1-e^{-s^{2}}\right)-\ln \left(1-\omega^{2} e^{-s^{2}}\right)\right\}}
\end{aligned}
$$

To evaluate this integral for large $j$, we rely on the saddle point approximation, which is valid as long as the condition

$$
\left(j+1+\frac{\gamma_{j}}{2}\right) \sqrt{1-\omega^{2}}>1
$$

is satisfied. To clearly illustrate the suppression we mentioned above, we write the Wilson coefficients in the form

$$
\bar{C}_{j}=c_{j}\left(\alpha_{s}(\mu), Q / \mu, \frac{\partial}{\partial \gamma}\right)\left(\frac{\mu^{2}}{2 Q^{2}}\right)^{\gamma_{j} / 2} \frac{\Gamma(j+1) \Gamma(j+2) \Gamma\left(2 j+4+\gamma_{j}\right) \omega^{j}}{\Gamma\left(j+2+\gamma_{j} / 2\right) \Gamma\left(j+3+\gamma_{j} / 2\right) \Gamma(2 j+3)} E_{j}\left(\omega \mid \gamma_{j}\right) .
$$


Consequently, we have the following normalization for the function $E_{j}\left(\omega=1 \mid \gamma_{j}\right)=1$ [cf. Eq. (3.38)]. For $|\omega|<1$, the approximation

$$
E_{j}\left(\omega \mid \gamma_{j}\right) \approx \frac{\sqrt{\pi} \sqrt{2\left(j+1+\gamma_{j} / 2\right) \sqrt{1-\omega^{2}}}}{2 \sqrt{1+\sqrt{1-\omega^{2}}}} \exp \left\{-\left(j+1+\frac{\gamma_{j}}{2}\right) \ln \left(1+\sqrt{1-\omega^{2}}\right)\right\}
$$

shows an exponential decrease for $(j+1) \ln \left(1+\sqrt{1-\omega^{2}}\right)>\sqrt{2}$. The value of $E_{j}\left(\omega \mid \gamma_{j}\right)$ is then smaller than $1 / 2$. Note that perturbative corrections due to the anomalous dimensions, which are positive and grow logarithmically with $j$, give a logarithmic enhancement of this behavior. In the case of rather small $|\omega|$, the suppression factor is proportional to $2^{-j-1} \sqrt{j+1}$, which affects even the lowest partial wave $j=2$. The suppression is already larger than $80 \%$ for given $j \geqslant 6$ as long as the inequality $1-|\omega|>4 /(j+1)^{2}$ is satisfied. Increasing $|\omega|$ will then abruptly increase the value of $E_{j}$ to reach the $E_{j}=1$ limit. To finish this general discussion, we estimate the partial wave that will be suppressed by a factor $\mathcal{E}=E_{j}(\omega) \leqslant 1 / e$ depending on $\omega$ :

$$
j+1 \simeq\left[\frac{-W_{-1}\left(-4 \mathcal{E}^{2}\left(1+\sqrt{1-\omega^{2}}\right) \ln \left(1+\sqrt{1-\omega^{2}}\right) /\left(\pi \sqrt{1-\omega^{2}}\right)\right)}{2 \ln \left(1+\sqrt{1-\omega^{2}}\right)}\right] \sim\left[\frac{-\ln \left(4 \mathcal{E}^{2} / \pi\right)}{4(1-|\omega|)}\right]
$$

where $W_{-1}(-x)$ is the product log function which is real valued in the region $0 \leqslant x \leqslant 1 / e$.

To estimate the contribution of the first few nonvanishing partial waves, we first consider the conformal moments $\left\langle\pi(P)\left|\mathcal{O}_{j j}\left(\mu_{f}\right)\right| \Omega\right\rangle^{\mathrm{red}}=6 N_{j} B_{j}$. The distribution amplitude vanishes at the end points [1] and from this behavior it follows that $N_{j} B_{j}$ vanishes at $j \rightarrow \infty$ :

$$
\begin{gathered}
\phi(x) \sim(1-x)^{\epsilon} \text { for } x \rightarrow 1 \Rightarrow 6 N_{j} B_{j} \sim j^{-\epsilon} \\
\text { for } j \rightarrow \infty \text { with } \epsilon>0 .
\end{gathered}
$$

We want to add that different nonperturbative estimates, based on a lattice calculation, sum rules, or a model calculation, give quite different values of $6 N_{2} B_{2}$ at a scale $Q \leqslant 1 \mathrm{GeV}$, varying from $\sim-1$ to $\sim+1$. Here the lower bound stems from a preliminary lattice calculation [64], while the upper one arises from sum rule estimates $[65,66]$ and is also compatible with previous lattice calculations (see [64] for references). There are other estimates that favor a rather small value of $B_{2}$. This suggests that the absolute size of the lowest few conformal moments $6 N_{j} B_{j}$ are of order 1 or even smaller. In the following estimates we consider them of order 1, which serves us as an upper bound for the contribution of the $j$ th partial wave to the transition form factor.

In the small $|\omega|$ region, i.e., $|\omega|<0.4$, the lowest partial wave contributes essentially. In LO the relative contributions of the second and fourth partial waves with respect to the first one for $|\omega|=0.2(0.4)$ are about $0.08 \%$ (2.3\%) and $0.004 \%(0.05 \%)$, respectively. The $\omega^{2}$ term of the zeroth partial wave varies in the same order as the relative correc- tion to the second partial wave, which is in addition suppressed by a relative factor of $2 / 3$. Thus, in the small $|\omega|$ region perturbative QCD provides us an (almost) parameterfree, factorization scheme independent prediction:

$$
\begin{aligned}
F_{\gamma \pi}(\omega, Q) \simeq & \frac{\sqrt{2} f_{\pi}}{3 Q^{2}} c_{0}\left(\alpha_{s}\left(\mu_{r}\right), Q / \mu_{r}\right)\left(1+\frac{\omega^{2}}{5}+O\left(\omega^{4}\right)\right) \\
& \text { for }|\omega|<0.4 .
\end{aligned}
$$

The phenomenological consequences are obvious, since this prediction is practically independent of $\omega$ and its logarithmical $Q^{2}$ dependence is governed only by the running of the coupling.

For intermediate values of $|\omega|$, defined as $0.4 \leqslant|\omega|$ $<0.8$, the second partial wave contributes between $2 \%$ and $13 \%$, while the fourth one is at least more than five times suppressed with respect to the second one. On increasing $|\omega|$ to the value 0.95 , the relative contributions of the second and fourth partial waves grow to $25 \%$ and $10 \%$, respectively, while the sixth (eighth) partial wave contributes at the $4 \%$ (2\%) level. It is illustrative to compare these numbers with the suppression arising in the limit $|\omega| \rightarrow 1$ in which the contribution amounts to $39 \%, 24 \%, 18 \%, 14 \%$ for $j=2,4,6,8$ partial waves, respectively.

As we have realized, only the first two nonvanishing partial waves are essential for an intermediate value of $|\omega|$. It could, therefore, be justified to employ the Taylor expansion of the hypergeometric functions at $\omega=0$ and hence the transition form factor reads in the $\overline{\mathrm{CS}}$ scheme

$$
\begin{aligned}
F_{\gamma \pi}(\omega, Q) \simeq & \frac{\sqrt{2} f_{\pi}}{3 Q^{2}}\left[c_{0}\left\{1+\frac{\omega^{2}}{5}+\frac{3 \omega^{4}}{35}\right\}+c_{2}\left(\frac{\mu^{2}}{Q^{2}}\right)^{\gamma_{2} / 2} \frac{2 \omega^{2}}{15}\left\{1+\frac{\left(8+\gamma_{2}\right)\left(6+\gamma_{2}\right)}{8\left(9+\gamma_{2}\right)} \omega^{2}\right\} 6 N_{2} B_{2}\left(\mu^{2}\right)+O\left(\omega^{6}\right)\right] \\
& \text { for } 0.4 \leqslant|\omega|<0.8,
\end{aligned}
$$


where the Wilson coefficient $c_{2}\left(\alpha_{s}(\mu), Q / \mu, \partial / \partial \gamma_{j}\right)$ is defined by Eq. (2.35) to NNLO accuracy and a consequent expansion in $\alpha_{s}$ should be done to this accuracy. For $|\omega|$ $=0.8$, the higher-order terms in $O\left(\omega^{6}\right)$ contribute at the $2 \%$ and $20 \%$ level for the $j=0$ and $j=2$ partial waves, respectively. These contributions can be reduced by a factor of 2 (4) by taking the order $\omega^{6}\left(\omega^{8}\right)$ corrections into account. For larger values of $|\omega|$, the convergence of the Taylor expansion at $\omega=0$ is rather slow for higher partial waves. For instance, to approximate the third nonvanishing partial wave at $|\omega|$ $=0.9$ to an accuracy of better than $10 \%$, one has to take into account the first ten nonvanishing terms, i.e., up to $O\left(\omega^{24}\right)$.

For ultralarge values of $|\omega|$, let us say $|\omega|>0.95$, partial waves with higher conformal spin start to contribute with increasing $|\omega|$. However, as we have discussed, as long as we do not reach the $|\omega| \rightarrow 1$ limit, there will be an exponential suppression for higher values of $j$. Note that this limit can never be reached in any experiment at an $e^{+} e^{-}$machine, where the mean value of the virtuality of the untagged photon is set by the electron mass and there are further kinematical restrictions arising from the detector geometry and kinematical cuts. Just for illustration, we would like to mention that, for $|\omega|=0.99$ and $|\omega|=0.999$, the contributions of the 12th and 38th partial waves are reduced by a factor $1 / e$ $\sim 0.37$ compared to their contributions in the limit $|\omega| \rightarrow 1$, while higher ones start to be exponentially suppressed, since the $(j+1) \ln \left(1+\sqrt{1-\omega^{2}}\right)>\sqrt{2}$ condition is satisfied.

We now present the general result of the photon-to-pion transition form factor for $|\omega|<1$ in its expanded form to the NNLO approximation. Notation analogous to that in Eq. (3.42) will be used, and the Taylor expansions in $\omega^{2}$ for the contributing terms of the first five nonvanishing partial waves are listed in Appendix D. At leading order the hypergeometrical functions can be expressed in terms of elementary arctanh functions, ${ }^{5}$ e.g.,

$$
\begin{aligned}
T_{0}^{(0)}(\omega)= & \frac{3}{2 \omega^{2}}\left[1-\left(1-\omega^{2}\right) \frac{\operatorname{arctanh}(\omega)}{\omega}\right], \\
T_{2}^{(0)}(\omega)= & \frac{7}{24 \omega^{2}}\left[15-13 \omega^{2}\right. \\
& \left.-\left(5-6 \omega^{2}+\omega^{4}\right) \frac{3 \operatorname{arctanh}(\omega)}{\omega}\right],
\end{aligned}
$$

and the expansion in $\omega$ is given in Eq. (D2). The radiative corrections for $\omega \neq 0$ depend on the factorization scheme even for the lowest partial waves. In comparison with Eqs. (3.43) and (3.44), here we will not include $\ln (2)$ terms in the factorization and renormalization $\operatorname{logs}$, i.e., instead of $2 Q^{2}$

\footnotetext{
${ }^{5}$ The result can be expressed in terms of ln functions by means of $\operatorname{arctanh}(\omega)=1 / 2 \ln [(1+\omega) /(1-\omega)]$.
}

we rather employ the underlying scale $Q^{2} \sim 1 / z^{2}$ of the OPE of two currents at the distance $z$. Thus, we have for the $\overline{\mathrm{MS}}$ scheme the NLO result (3.16)

$$
T_{j}^{(1)}\left(\omega, Q / \mu_{f}\right)=C_{F}\left[T_{F, j}^{(1)}(\omega)+T_{j}^{(0)}(\omega) \ln \left(\frac{Q^{2}}{\mu_{f}^{2}}\right) v_{j}\right],
$$

and analogously for the CS scheme. Note that in comparison to this notation the definitions of conformal moments in the $|\omega| \rightarrow 1$ limit, Eqs. (3.32) and (3.45), differ by a $\ln (2)$-proportional term:

$$
\begin{gathered}
\lim _{\omega \rightarrow 1} T_{F, j}^{(1)}(\omega)=T_{F, j}^{(1)}+\ln (2) T_{j}^{(0)} v_{j}, \\
\lim _{\omega \rightarrow 1} T_{F, j}^{\mathrm{CS}(1)}(\omega)=T_{F, j}^{\mathrm{CS}(1)}+\ln (2) T_{j}^{(0)} v_{j} .
\end{gathered}
$$

Expanding the $T^{(0)}(\omega) \operatorname{LN}^{i}(\omega)$ terms in Eq. (3.18) provides after convolution with the corresponding kernels the desired result in the $\overline{\mathrm{MS}}$ scheme (D8). In the considered order of $\omega^{2}$, it coincides with the result of Ref. [30]. The result in the CS scheme can be easily derived by expanding Eq. (2.18) in order $\alpha_{s}$ :

$$
T_{F, j}^{\mathrm{CS}(1)}(\omega)=\left[c^{(1)}-v_{j} s_{j}^{(1)}(\omega)\right] T_{j}^{(0)}(\omega)
$$

where $c^{(1)}$ and $v_{j}$ are given by Eqs. (3.41) and (3.34), respectively, and $s_{j}^{(i)}(\omega)$ is defined by the expansion

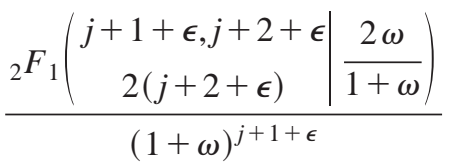

$$
\begin{aligned}
& =\left[1+s_{j}^{(1)}(\omega) \epsilon+\frac{1}{2} s_{j}^{(2)}(\omega) \epsilon^{2}+O\left(\epsilon^{3}\right)\right] \\
& \times \frac{\left.{ }_{2} F_{1}\left(\begin{array}{c}
j+1, j+2 \\
2(j+2)
\end{array}\right) \frac{2 \omega}{1+\omega}\right)}{(1+\omega)^{j+1}} .
\end{aligned}
$$

The corresponding expansions of $s_{j}^{(1)}$ and $s_{j}^{(2)}$ in $\omega^{2}$ are given for $j=2,4,6,8$ (because of current conservation $v_{0}=0$ and, consequently, the $j=0$ term does not contribute) given in Eqs. (D5) and (D7), respectively. The NNLO contribution in the CS scheme we write analogously to Eq. (3.44) as 


$$
\begin{aligned}
T_{j}^{\mathrm{CS}(2)}(\omega)= & C_{F}\left\{C_{F} T_{F, j}^{\mathrm{CS}(2)}(\omega)-\frac{\beta_{0}}{2} T_{\beta, j}^{(2)}(\omega)-\left(C_{F}-\frac{1}{2} C_{A}\right) T_{G, j}^{\mathrm{CS}(2)}(\omega)\right. \\
& +\ln \left(\frac{Q^{2}}{\mu_{f}^{2}}\right) T_{j}^{(0)}(\omega)\left\{C_{F}\left[v_{F, j}+c_{j}^{(1)} v_{j}-v_{j}^{2} s_{j}^{(1)}(\omega)+\frac{v_{j}^{2}}{2} \ln \left(\frac{Q^{2}}{\mu_{f}^{2}}\right)\right]-\frac{\beta_{0}}{2} v_{\beta, j}^{\mathrm{CS}, \Sigma}(\omega)-\left(C_{F}-\frac{1}{2} C_{A}\right) v_{G, j}\right\} \\
& \left.+\frac{\beta_{0}}{2} \ln \left(\frac{Q^{2}}{\mu_{r}^{2}}\right) T_{j}^{\mathrm{CS}(1)}\left(\omega, Q / \mu_{f}\right)-\frac{\beta_{0}}{4} \ln ^{2}\left(\frac{Q^{2}}{\mu_{f}^{2}}\right) T_{j}^{(0)}(\omega) v_{j}\right\}
\end{aligned}
$$

In comparison to the definitions in Eqs. (3.46), (3.47), and (3.33), one has again to take care of $\ln (2)$ terms by means of the following correspondences:

$$
\begin{aligned}
\lim _{\omega \rightarrow 1} T_{F, j}^{\mathrm{CS}(2)}(\omega)= & T_{F, j}^{\mathrm{CS}(2)}+\ln (2) T_{j}^{(0)}\left[v_{F, j}+c_{j}^{(1)} v_{j}\right. \\
& \left.-v_{j}^{2}\left(s_{j}^{(1)}(\omega=1)+\frac{1}{2} \ln (2)\right)\right], \\
\lim _{\omega \rightarrow 1} T_{G, j}^{\mathrm{CS}(2)}(\omega)= & T_{G, j}^{\mathrm{CS}(2)}+\ln (2) T_{j}^{(0)} v_{G, j}, \\
\lim _{\omega \rightarrow 1} T_{\beta, j}^{(1)}(\omega)= & T_{\beta, j}^{(1)}+\ln (2)\left[T_{j}^{(0)} v_{\beta, j}^{\mathrm{CS}, \Sigma}-T_{F, j}^{\mathrm{CS}(1)}\right. \\
& \left.-\frac{1}{2} \ln (2) T_{j}^{(0)} v_{j}\right],
\end{aligned}
$$

and similarly for the quantities in the $\overline{\mathrm{CS}}$ scheme. Here we have also employed the identity (D7) For $T_{F, j}^{\mathrm{CS}(2)}(\omega)$ and $T_{G, j}^{\mathrm{CS}(2)}(\omega)$, the expansion of the Wilson coefficients (2.18) gives

$$
\begin{aligned}
T_{F, j}^{\mathrm{CS}(2)}(\omega)= & T_{j}^{(0)}(\omega)\left\{c_{F, j}^{(2)}-\left(c_{j}^{(1)} v_{j}+v_{F, j}\right) s_{j}^{(1)}(\omega)\right. \\
& \left.+\frac{v_{j}^{2}}{2} s_{j}^{(2)}(\omega)\right\}, \\
T_{G, j}^{\mathrm{CS}(2)}(\omega)= & T_{j}^{(0)}(\omega)\left\{c_{G, j}^{(2)}-v_{G, j} s_{j}^{(1)}(\omega)\right\} .
\end{aligned}
$$

The conformal moments proportional to $\beta_{0}$ are obtained from the $\overline{\mathrm{MS}}$ result given by Eq. (3.20). The expansion in $\omega^{2}$ of the term $T_{\beta, j}^{(2)}$ is given by Eq. (D9). As in the $|\omega|=1$ case [see the discussion of expression (3.35)], we define the conformal moments of $T^{(0)}(\omega) \otimes\left[v_{\beta}\right]_{+}$by $T_{j}^{(0)}(\omega) v_{\beta, j}^{\Sigma}(\omega)$. Note that the "effective" conformal moment $v_{\beta, j}^{\Sigma}$ now depends on $\omega$ and its expansion in $\omega^{2}$ is given in Eq. (D10). Analogously to Eq. (3.48), $v_{\beta, j}^{\mathrm{CS}, \Sigma}(\omega)$ is provided by

$$
v_{\beta, j}^{\mathrm{CS}, \Sigma}(\omega)=v_{\beta, j}^{\Sigma}(\omega)+\frac{T_{F, j}^{\mathrm{CS}(1)}(\omega)-T_{F, j}^{(1)}(\omega)}{T_{j}^{(0)}(\omega)},
$$

and the expansion can easily be obtained by means of Eqs. (3.62), (D5), and (D8).

The $\beta_{0}$-proportional NNLO terms in the $\overline{\mathrm{CS}}$ scheme are obtained by making the replacements

$$
\begin{aligned}
T_{\beta, j}^{(2)}(\omega) \rightarrow & T_{\beta, j}^{\overline{\mathrm{CS}}(2)}(\omega)=T_{j}^{(0)}(\omega)\left\{c_{\beta, j}^{(2)}-v_{\beta, j} s_{j}^{(1)}(\omega)\right\} \\
& \text { and } v_{\beta, j}^{\mathrm{CS}, \Sigma} \rightarrow v_{\beta, j}^{\overline{\mathrm{CS}}}=v_{\beta, j} T_{j}^{(0)}(\omega),
\end{aligned}
$$

while the other terms remain the same as in the CS scheme.

\section{RADIATIVE CORRECTIONS TO THE PHOTON- TO-PION TRANSITION FORM FACTOR}

This section is devoted to a model independent study of radiative corrections to the pion-to-photon transition form factor in the case of one quasireal photon $(|\omega| \rightarrow 1)$ and in the small and intermediate $|\omega|$ regions. We also illustrate how the perturbative QCD approach to exclusive processes can be tested in a novel way by a sum rule and how the two lowest nontrivial conformal moments of the pion distribution amplitude could be extracted from experimental data in the intermediate $|\omega|$ region.

In Sec. IV A we briefly review the features of the radiative corrections to the first few conformal moments of the hard-scattering amplitude in the $\overline{\mathrm{MS}}$ and CS schemes to NLO. We point out that asymptotic formulas with respect to the conformal spin $j+1$ provide a very good approximation of the moments in question for a rather low value of $j \geqslant 4$. As a by-product, we propose a simple method for reconstructing the amplitude from its conformal moments, which is outlined in Appendix E.

In Sec. IV B we present the numerical values of the NNLO corrections to the first five non-vanishing conformal moments of the hard-scattering amplitude in the CS and $\overline{\mathrm{CS}}$ schemes. We point out their general features and discuss different possibilities for treating the $\beta_{0}$ terms. In particular, we consider the lowest conformal partial wave and compare its contribution to the photon-to-pion transition form factor with experimental data. We study the influence of radiative corrections to the sum rule and show that higher-order corrections will interfere only slightly in the extraction of the two lowest nontrivial conformal moments of the distribution amplitude. 
TABLE I. First moments of $T_{F, j}^{(0)}, T_{F, j}^{(1)}$, and $T_{F, j}^{C S(1)}$ for $|\omega|=1$ with $\mu_{f}^{2}=2 Q^{2}$ and for $|\omega|=0.8$ with $\mu_{f}^{2}=Q^{2}$.

\begin{tabular}{lccccccccccc}
\hline \hline & $j$ & 0 & 2 & 4 & 6 & 8 & 10 & 12 & 14 & 16 & 18 \\
\hline$|\omega|=1$ & $T^{(0)}$ & 1.5 & 0.58 & 0.37 & 0.27 & 0.21 & 0.17 & 0.15 & 0.13 & 0.11 & 0.10 \\
$\mu_{f}^{2}=2 Q^{2}$ & $T_{F, j}^{(1)}$ & -3.75 & 1.2 & 2.14 & 2.38 & 2.42 & 2.39 & 2.33 & 2.26 & 2.19 & 2.12 \\
& $T_{F, j}^{C S(1)}$ & -2.25 & 1.91 & 2.52 & 2.58 & 2.51 & 2.41 & 2.3 & 2.19 & 2.09 & 2 \\
\hline$|\omega|=0.8$ & $T^{(0)}$ & 1.19 & 0.15 & 0.03 & 0.01 & - & - & - & - & - & - \\
$\mu_{f}^{2}=Q^{2}$ & $T_{F, j}^{(1)}$ & -2.02 & 0.14 & 0.1 & 0.03 & 0.01 & - & - & - & - & - \\
& $T_{F, j}^{\mathrm{CS}(1)}$ & -1.78 & 0.16 & 0.1 & 0.03 & 0.01 & - & - & - & - & - \\
\hline \hline
\end{tabular}

\section{A. Features of radiative corrections at NLO}

Let us first compare the NLO corrections in the $\overline{\mathrm{MS}}$ and CS schemes for $|\omega| \rightarrow 1$. The first ten nonvanishing moments $T_{F, j}^{(1)}$ and $T_{F, j}^{\mathrm{CS}(1)}$, given by Eqs. (3.32) and (3.45), are shown in Table I. From this table we realize that the main difference between the two schemes is in the first two moments, which differ by about $50 \%$, and in a seemingly faster decrease of the moments in the CS scheme for large $j$. Indeed, in the large $j$ asymptotics the leading terms are

$$
\begin{aligned}
T_{F, j}^{(1)}= & \frac{2 j+3}{(j+1)(j+2)}\left\{2 S_{1}(1+j)^{2}-\frac{9}{2}+O\left((j+2)^{-1}\right)\right\}, \\
T_{F, j}^{\mathrm{CS}(1)}= & \frac{2 j+3}{(j+1)(j+2)}\left\{S_{1}(1+j)\left[S_{1}(1+j)+\frac{3}{2}+4 \ln (2)\right]\right. \\
& \left.-\frac{9}{2}-\zeta(2)-3 \ln (2)+O\left((j+2)^{-1}\right)\right\} .
\end{aligned}
$$

Taking into account the large $j$ asymptotics of the $S_{1}$ functions, given by

$$
\lim _{j \rightarrow \infty} S_{1}(1+j)=\ln (2+j)+\gamma_{\mathrm{E}}
$$

the ratio $T_{F, j}^{\mathrm{CS}(1)} / T_{F, j}^{(1)}$ slowly approaches $1 / 2$. The difference is caused by the (infinite) resummation of off-diagonal terms in the $\overline{\mathrm{MS}}$ scheme. The asymptotic formulas (4.1) have a relative error of less than $2 \%$ already for $j \geqslant 4$. Thus, by knowing a few lowest moments and their asymptotics we gain a complete insight into the radiative corrections for $|\omega|=1$. In Appendix E we use this result to make an approximate reconstruction of the hard-scattering amplitude in the momentum fraction representation from its conformal moments. The consequence of the logarithmic behavior in Eq. (4.2) is obviously an increase of radiative corrections with growing conformal spin. It is shown in Table II that already for $j$ $=8$ radiative corrections are of the size of $80 \%$ for $\alpha_{s} / \pi$ $\simeq 0.1$ (i.e., $\mu_{r} \approx 2 \mathrm{GeV}$ for one-loop $\alpha_{s}$ with $n_{f}=3$ ). From this point of view, one might conclude that perturbation theory breaks down for rather large values of $j$. Fortunately, higher conformal spin contributions are suppressed by the nonperturbative input [see Eq. (3.56)] and so perturbative QCD remains applicable. In the photon-to-pion transition form factor there might also be a cancellation of the lowest partial wave with the remainder, which is due to their relative minus sign. Of course, the net contribution of radiative corrections depends on the model of the distribution amplitude itself.

With decreasing $|\omega|$, higher conformal partial waves are starting to be exponentially suppressed, and, as we have shown in Sec. III D, radiative corrections logarithmically enhance this suppression. Also note that off-diagonal contributions to each partial wave, which are relatively suppressed by powers of $\omega^{2}$ with respect to the diagonal ones, are becoming small. If we approach the equal virtuality case, i.e., $\omega$ $=0$, only a factorization-scheme-independent constant, arising from the lowest partial wave, will survive. Thus, by decreasing $|\omega|$ the differences between the $\overline{\mathrm{MS}}$ and CS schemes must be washed out. In Table I we illustrate these effects for $|\omega|=0.8$. For the two lowest nonvanishing partial waves the difference between these two schemes is reduced to about $\pm 14 \%$ and for higher ones below $2 \%$. In the CS scheme also the contributions from the functions $s^{(i)}(\omega)$ $=O\left(\omega^{2}\right)[$ cf. Eq. (3.63)] are power suppressed. So one expects from Eq. (3.62) that the radiative corrections due to the normalization factors $c_{j}^{(i)}$ are the essential ones, but with one exception. Since the coefficient $c_{2}^{(1)}$ is relatively small compared to the anomalous dimension $\gamma_{2}^{(1)}, O\left(\omega^{2}\right)$ corrections remain important for the second partial wave in the intermediate $|\omega|$ region.

TABLE II. The ratio of NLO to LO and NNLO to NLO radiative corrections in units of $\alpha_{s} / \pi$ for $|\omega|=1$ and $\mu_{r}^{2}=\mu_{f}^{2}=2 Q^{2}$ in the $\overline{\mathrm{MS}}$, CS, and $\overline{\mathrm{CS}}$ schemes.

\begin{tabular}{lrcccc}
\hline \hline$j$ & $\frac{T_{j}^{(1)}}{2 T_{j}^{(0)}}$ & $\frac{T_{j}^{(2)}}{2 T_{j}^{(1)}}$ & $\frac{T_{j}^{\mathrm{CS}(1)}}{2 T_{j}^{(0)}}$ & $\frac{T_{j}^{\mathrm{CS}(2)}}{2 T_{j}^{\mathrm{CS}(1)}}$ & $\frac{T_{j}^{\overline{\mathrm{CS}}(2)}}{2 T_{j}^{\mathrm{CS}}(1)}$ \\
\hline 0 & -1.67 & - & -1 & 7.23 & 5.14 \\
2 & 1.37 & - & 2.18 & 4.54 & 4.13 \\
4 & 3.88 & - & 4.58 & 7.44 & 6.11 \\
6 & 5.92 & - & 6.42 & 9.21 & 7.39 \\
8 & 7.64 & - & 7.93 & 10.56 & 8.39 \\
\hline \hline
\end{tabular}


TABLE III. The first five nonvanishing Wilson coefficients appearing in the perturbative expansions of $T_{j}^{\mathrm{CS}}$ and $T_{j}^{\overline{\mathrm{CS}}}$ with respect to $\alpha_{s} / \pi$ at NNLO accuracy for $|\omega|=1$. The results are obtained employing $\mu_{f}^{2}$ $=\mu_{r}^{2}=2 Q^{2}$.

\begin{tabular}{llcrcrc}
\hline \hline$j$ & $T_{j}^{(0)}$ & $\frac{C_{F}}{2} T_{F, j}^{\mathrm{CS}(1)}$ & $\frac{C_{F}^{2}}{4} T_{F, j}^{\mathrm{CS}(2)}$ & $-\frac{C_{F}\left(2 C_{F}-C_{A}\right)}{8} T_{G, j}^{\mathrm{CS}(2)}$ & $-\frac{C_{F} \beta_{0}}{8} T_{\beta, j}^{(2)}$ & $-\frac{C_{F} \beta_{0}}{8} T_{\beta, j}^{\overline{\mathrm{CS}}(2)}$ \\
\hline 0 & 1.5 & -1.5 & 1.42 & -0.04 & -12.23 & -9.09 \\
2 & 0.58 & 1.27 & -2.28 & -0.53 & 8.58 & 8.06 \\
4 & 0.37 & 1.68 & -0.46 & -0.60 & 13.56 & 11.33 \\
6 & 0.27 & 1.72 & 1.25 & -0.58 & 15.17 & 12.06 \\
8 & 0.21 & 1.67 & 2.54 & -0.55 & 15.68 & 12.05 \\
\hline \hline
\end{tabular}

\section{B. Predictions to NNLO accuracy}

\section{The quasireal photon limit}

We now turn to the discussion of NNLO effects in the CS and $\overline{\mathrm{CS}}$ schemes starting with the limit $|\omega| \rightarrow 1$. In Table III we present the numerical values of the Wilson coefficients $T_{F, j}^{\mathrm{CS}(2)}, T_{G, j}^{\mathrm{CS}(2)}, T_{\beta, j}^{\mathrm{CS}(2)}=T_{\beta, j}^{(2)}$, and $T_{\beta, j}^{\overline{\mathrm{CS}}(2)}$ corresponding to Eqs. (3.46), (3.47), (3.33), and (3.49), respectively. The values of $T_{F, j}^{\mathrm{CS}(2)}, T_{G, j}^{\mathrm{CS}(2)}$, and $T_{\beta, j}^{\overline{\mathrm{CS}}(2)}$ were obtained by means of the NNLO result for the deep inelastic scattering structure function $g_{1}$ [43].

Let us investigate in more detail the contribution of the lowest partial wave to the transition form factor, which is scheme dependent for $\omega \neq 0$ and for $|\omega|=1$ reads: in the $\overline{\mathrm{MS}}$ scheme,

$$
\begin{aligned}
F_{\gamma \pi}(Q)= & \frac{\sqrt{2} f_{\pi}}{2 Q^{2}}\left\{1-\frac{5}{3} \frac{\alpha_{s}\left(\mu_{r}\right)}{\pi}+\frac{\alpha_{s}^{2}\left(\mu_{r}\right)}{\pi^{2}}\{\ldots\right. \\
& \left.-\frac{\beta_{0}}{2}\left[-1.811+\frac{5}{6} \ln \left(\frac{2 Q^{2}}{\mu_{r}^{2}}\right)-0.285 \ln \left(\frac{2 Q^{2}}{\mu_{f}^{2}}\right)\right]\right\} \\
& \left.+O\left(\alpha_{s}^{3}\right)\right\},
\end{aligned}
$$

in the CS scheme,

$$
\begin{aligned}
F_{\gamma \pi}(Q)= & \frac{\sqrt{2} f_{\pi}}{2 Q^{2}}\left\{1-\frac{\alpha_{s}\left(\mu_{r}\right)}{\pi}+\frac{\alpha_{s}^{2}\left(\mu_{r}\right)}{\pi^{2}}\{0.917\right. \\
& \left.-\frac{\beta_{0}}{2}\left[-1.811+\frac{1}{2} \ln \left(\frac{2 Q^{2}}{\mu_{r}^{2}}\right)+0.048 \ln \left(\frac{2 Q^{2}}{\mu_{f}^{2}}\right)\right]\right\} \\
& \left.+O\left(\alpha_{s}^{3}\right)\right\},
\end{aligned}
$$

and in the $\overline{\mathrm{CS}}$ scheme,

$$
\begin{aligned}
F_{\gamma \pi}(Q)= & \frac{\sqrt{2} f_{\pi}}{2 Q^{2}}\left\{1-\frac{\alpha_{s}\left(\mu_{r}\right)}{\pi}+\frac{\alpha_{s}^{2}\left(\mu_{r}\right)}{\pi^{2}}\{0.917\right. \\
& \left.\left.-\frac{\beta_{0}}{2}\left[-1.347+\frac{1}{2} \ln \left(\frac{2 Q^{2}}{\mu_{r}^{2}}\right)\right]\right\}+O\left(\alpha_{s}^{3}\right)\right\} .
\end{aligned}
$$

For $\alpha_{s}\left(\mu_{r}^{2}\right) / \pi=0.1$, the ratio of the NLO to the LO contribution is $-17 \%$ in the $\overline{\mathrm{MS}}$ scheme and $-10 \%$ in the CS scheme. This difference arises from the fact that in the $\overline{\mathrm{MS}}$ scheme off-diagonal terms of the hard-scattering amplitude are resummed. In Eq. (4.3) we see that the $\ln \left(2 Q^{2} / \mu_{f}^{2}\right)$ term is rather small compared to the $\ln \left(2 Q^{2} / \mu_{r}^{2}\right)$ one. This is even more the case in the $\mathrm{CS}$ scheme, while in the $\overline{\mathrm{CS}}$ scheme the $\ln \left(2 Q^{2} / \mu_{f}^{2}\right)$ term vanishes completely, since all off-diagonal entries in the NLO evolution have been removed. The sign alternating series of the $\beta_{0}$-nonproportional terms is due to the Sudakov effect; see Ref. [23] for a detailed discussion.

Since factorization-scale-changing effects in the hardscattering amplitude are quite small for the lowest partial wave, and since they will be compensated by the evolution of the nonperturbative part (see Sec. II D), we set $\mu_{f}^{2}=2 Q^{2}$ in the following and discuss the scale setting of the residual $\mu_{r}$ dependence. First, let us equate $\mu_{r}^{2}=2 Q^{2}$ :

$$
F_{\gamma \pi}(Q)=\frac{\sqrt{2} f_{\pi}}{2 Q^{2}}\left[1-\frac{\alpha_{s}\left(2 Q^{2}\right)}{\pi}-\left\{\begin{array}{l}
7.23 \\
5.14
\end{array}\right\} \frac{\alpha_{s}^{2}\left(2 Q^{2}\right)}{\pi^{2}}\right.
$$

$$
\left.+O\left(\alpha_{s}^{3}\right)\right] \text { for the } \begin{cases}\mathrm{CS} & \text { scheme } \\ \overline{\mathrm{CS}} & \text { scheme. }\end{cases}
$$

Hence, for $\alpha_{s}\left(\mu_{r}^{2}\right) / \pi=0.1$, the ratio of the NNLO to the LO contribution is $-7.2 \%$ and $-5.1 \%$, and the ratio of the NNLO to the NLO contribution (the measure of the convergence of the perturbative QCD expansion) is $\approx 70 \%$ and $\approx 50 \%$, in the $\mathrm{CS}$ and $\overline{\mathrm{CS}}$ schemes, respectively.

The main part of these rather large NNLO contributions arises from the $\beta_{0}$-proportional term. Owing to the offdiagonal parts, it is larger by about a factor of 2 in the CS scheme than in the $\overline{\mathrm{CS}}$ scheme. It is appealing to resum this large contribution by the Brodsky-Lepage-McKenzie (BLM) proposal [67] (for application to exclusive processes, see also 
TABLE IV. Ratio $a_{\mathrm{BLM}}=\mu_{\mathrm{BLM}}^{2} / 2 Q^{2}$ of the BLM scale squared to $2 Q^{2}$, and the ratio of the NNLO to the NLO coefficient in units of $\alpha_{s} / \pi$ for $\mu_{r}^{2}=\mu_{\mathrm{BLM}}^{2}, \mu_{f}^{2}=2 Q^{2}$, and $|\omega|=1$.

\begin{tabular}{cccccc}
\hline \hline$j$ & $a_{\mathrm{BLM}}^{\overline{\mathrm{MS}}}$ & $a_{\mathrm{BLM}}^{\mathrm{CS}}$ & $a_{\mathrm{BLM}}^{\overline{\mathrm{CS}}}$ & $\frac{T_{j}^{(2)}}{2 T_{j}^{(1)}}$ & $\frac{T_{j}^{\mathrm{CS}(2)}}{2 T_{j}^{\mathrm{CS}(1)}}=\frac{T_{j}^{\overline{\mathrm{CS}}(2)}}{2 T_{j}^{\overline{\mathrm{CS}}(1)}}$ \\
\hline 0 & $1 / 8.79$ & $1 / 37.43$ & $1 / 14.78$ & - & -0.92 \\
2 & $1 / 120.08$ & $1 / 20.1$ & $1 / 16.76$ & - & -2.22 \\
4 & $1 / 68.73$ & $1 / 36.17$ & $1 / 20.05$ & - & -0.63 \\
6 & $1 / 70.3$ & $1 / 50.41$ & $1 / 22.52$ & - & 0.39 \\
8 & $1 / 75.29$ & $1 / 64.4$ & $1 / 24.54$ & - & 1.19 \\
\hline
\end{tabular}

[68]), in which all terms proportional to $\beta$ are absorbed in the coupling by the scale setting $\mu_{r}=\mu_{\mathrm{BLM}}$ :

$$
F_{\gamma \pi}(Q)=\frac{\sqrt{2} f_{\pi}}{2 Q^{2}}\left\{1-\frac{\alpha_{s}\left(\mu_{\mathrm{BLM}}\right)}{\pi}+0.92 \frac{\alpha_{s}^{2}\left(\mu_{r}\right)}{\pi^{2}}+O\left(\alpha_{s}^{3}\right)\right\}
$$

with

$$
\mu_{\mathrm{BLM}}^{2}=2 Q^{2}\left\{\begin{array}{l}
1 / 37.43 \\
1 / 14.78
\end{array}\right\} \text { for the } \begin{cases}\mathrm{CS} & \text { scheme } \\
\overline{\mathrm{CS}} & \text { scheme. }\end{cases}
$$

The ratio of the NNLO to the NLO coefficient is now only -1 and reflects the Sudakov effect in the conformal theory. However, as we realize, combining the COPE result with the $\overline{\mathrm{MS}}$ result of the $\beta_{0}$-proportional piece induces a rather low scale. For instance, for $2 Q^{2}=4 \mathrm{GeV}^{2}$ we have $\mu_{\mathrm{BLM}}^{2}$ $\sim 0.1 \mathrm{GeV}^{2}$ in the $\mathrm{CS}$ scheme and hence nonperturbative behavior of the coupling is needed. If we completely remove the off-diagonal terms, the BLM scale squared is enlarged by a factor of 2.7 and is now closer to that in the $\overline{\mathrm{MS}}$ scheme [10] given in Table IV. What one is actually doing here is to combine perturbative QCD with speculations about the nonperturbative behavior of the QCD coupling and so, strictly speaking, one is leaving the perturbative ground on which the whole analysis was based. However, one advantage of this proposal is that the result predicted by conformal symmetry is recovered if we consequently assume a hypothetical fixed point of the $\beta$ function during our considerations. What we in fact do by the freezing of the coupling is to assume that this nonperturbative fixed point is at $Q^{2}=0$.

In Fig. 1 we compare the experimental data from the CLEO experiment with the prediction arising from the lowest conformal moment, the only one that survives in the asymptotic limit $Q^{2} \rightarrow \infty$. Without further considerations, we assume, as has also been done in the method of data extraction employed (see Sec. 3 of Ref. [18]), that the quasireal photon limit has been reached. The prediction for the asymptotically large $Q^{2}$ in this $|\omega| \rightarrow 1$ limit is displayed as a dotted line. As we have discussed, radiative corrections reduce the size of this prediction for realistic values of $Q^{2}$. The dash-dotted line represent the NLO and the solid line the NNLO prediction for the standard $\overline{\mathrm{MS}}$ definition of the cou-

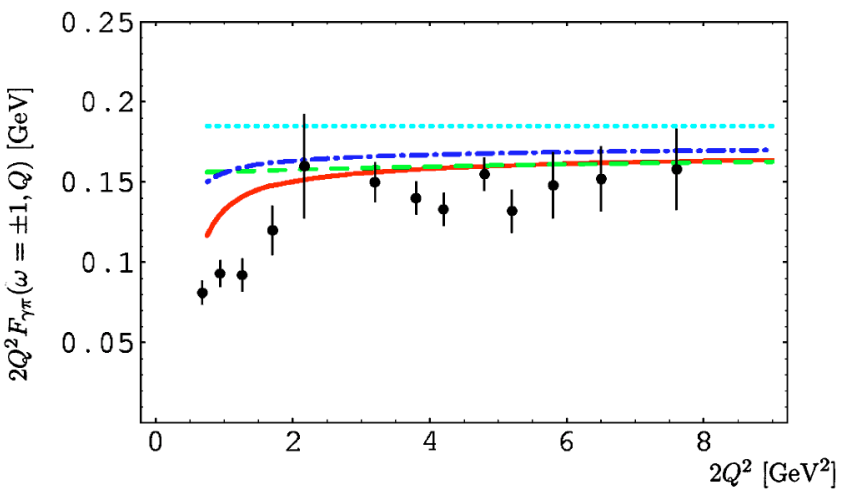

FIG. 1. The contribution of the first partial wave to the scaled photon-pion transition form factor $2 Q^{2} F_{\gamma \pi}(\omega= \pm 1, Q)$ is shown in LO (dotted), NLO (dash-dotted), and NNLO (solid and dashed) accuracy for the $\overline{\mathrm{CS}}$ scheme. The renormalization scale has been set to $\mu_{r}^{2}=2 Q^{2}$ (solid) and to the BLM scale $\mu_{r}^{2}=2 Q^{2} / 14.7$ (dashed). The data are taken from Refs. $[17,18]$.

pling in the corresponding approximation with the normalization $\alpha_{s}\left(\mu_{r}=M_{Z}\right)=0.118$. The dashed line is the prediction for the BLM scale setting and accumulates nonperturbative effects by freezing the coupling to $\alpha_{s}\left(\mu_{r}\right.$ $=0)=0.6$ by adding an effective gluon mass. Let us note that the scale setting ambiguities at $2 Q^{2}=1 \mathrm{GeV}^{2}$ are of the order of $20 \%$ if we vary the renormalization scale $\mu_{r}^{2}$ from $0.5 \mathrm{GeV}^{2}$ to $2 \mathrm{GeV}^{2}$. This ambiguity can be further reduced by going to higher orders in $\alpha_{s}$.

This comparison shows that theoretical uncertainties due to higher-order radiative corrections and scale-setting ambiguities are much smaller than the error of the experimental data. At larger values of $Q^{2}$ there is no significant contribution of higher partial waves. However, there is a significant discrepancy of the results in Fig. 1 in the region $0.5 \mathrm{GeV}^{2}$ $<2 Q^{2}<2 \mathrm{GeV}^{2}$, which may indicate the presence of higher partial waves. Since evolution effects in this kinematical region are rather strong, we could employ them to pin down the size of higher partial waves. However, the question arises: Can we in this kinematical window rely on the leading twist result?

By considering the size of power suppressed contributions, we will now argue that the answer to this question is positive. Since the (local) matrix elements of any operator appearing in the OPE can be built with only the momentum four-vectors $P$, Lorentz covariance immediately tells us that power suppressed contributions are of even power in $Q$. The only dimensional parameters that can appear are the mass of the pion $m_{\pi} \sim 0.14 \mathrm{GeV}$ and the QCD scale parameter $\Lambda$ $\sim 0.2 \mathrm{GeV}$. Assuming that multipartonic correlation functions will not have a strong numerical enhancement, we expect that the contributions proportional to $m_{\pi}^{2}$ will provide a relative correction of the order of $0.02 \mathrm{GeV}^{2} / 2 Q^{2}$. The size of the remaining nonperturbative corrections arises from ambiguities in summing the perturbative series and can be estimated in the framework of renormalons. In the conformal scheme, we might again borrow the results from the analysis of the coefficient of the structure function $g_{1}$, which gives for the lowest moment an uncertainty of a similar size (see 
Ref. [69] and references therein). Thus, we might conclude that the nonperturbative effects to the contribution of the zero partial wave are smaller than $10 \%$ at a scale of $2 Q^{2}$ $\sim 1 \mathrm{GeV}^{2}$. Certainly, this rather optimistic speculation should be confronted with other methods used. The lowest conformal moments of twist-4 quark-gluon-quark operators have been obtained by means of QCD sum rules [70,71]. Here it was found that, relative to $m_{\pi}^{2}$, a certain matrix element is numerically enhanced by a factor of 10 . This certainly would strike our point of view and indicate that the so-called Wandzura-Wilczek approximation, in which higher multiparton correlations are neglected, fails. Renormalon induced corrections have been studied in a model-dependent way in Ref. [8], where their relative size was estimated to be $0.2 \mathrm{GeV}^{2} / 2 Q^{2}$. Note, however, that in this analysis excitations of higher conformal partial waves have been included. Making it short, we stress that the estimate of higher-twist contributions has to be made in a consistent framework that is set by the scheme in which one started. Combining estimates from different approaches is a popular but rather awkward procedure.

In this process with a quasireal photon higher partial waves are summed. Even if the $|\omega| \rightarrow 1$ limit is not reached in the experiment, a rather large number of terms will contribute. Without any knowledge about the shape of the distribution amplitude, it is a rather vague assumption to truncate this series by hand to extract the values of the lowest partial waves from the normalization of the pion-to-photon transition form factor. Figure 1 clearly shows that the dominant contribution, at least for $2 Q^{2}>2 \mathrm{GeV}^{2}$, arises from the lowest partial wave, and the remainder is small. The fact that the contributions of higher partial waves cancel each other is not excluded, and it remains a claim that the asymptotic shape of the distribution amplitude is established by experimental data. In principle, one can gain more information on the remainder if one also employs the evolution of the distribution amplitude. However, even if rather high-precision data are available, the deconvolution problem is not easy to solve. As we have already mentioned, at NLO the perturbative correction will increase with growing conformal spin. The same tendency can be read off from Table II also in NNLO, where the $\beta_{0}$-proportional term is the dominant one. This is also reflected by the decrease of the BLM scale as shown in Table IV, where we can also see that the remaining corrections at NNLO are moderate. Note that the BLM scale is rather low for $2 \leqslant j$ in the $\overline{\mathrm{MS}}$ and CS schemes, which is due to off-diagonal terms.

\section{What can we learn from the small and intermediate $|\omega|$ regions?}

As was clearly spelt out in Ref. [30] and explained in a more general way in Sec. III D, the small $|\omega|$ region is suitable for a novel test of the perturbative QCD approach to the class of exclusive light-cone dominated processes. As we noted in Sec. IV A, for decreasing $|\omega|$ the differences between different schemes will decrease too. This is illustrated for $|\omega|=0.8$ in Tables V and VI. For the lowest (second) partial wave we have about a $-40 \%(10 \%)$ effect at NNLO
TABLE V. Same as Table II for $|\omega|=0.8$ and $\mu_{r}^{2}=\mu_{f}^{2}=Q^{2}$.

\begin{tabular}{cccccc}
\hline \hline & $\frac{T_{j}^{(1)}}{2 T_{j}^{(0)}}$ & $\frac{T_{j}^{(2)}}{2 T_{j}^{(1)}}$ & $\frac{T_{j}^{\mathrm{CS}(1)}}{2 T_{j}^{(0)}}$ & $\frac{T_{j}^{\mathrm{CS}(2)}}{2 T_{j}^{\mathrm{CS}(1)}}$ & $\frac{T_{j}^{\mathrm{CS}(2)}}{2 T_{j}^{\mathrm{CS}(1)}}$ \\
\hline 0 & -1.14 & - & -1 & 4.21 & 3.58 \\
2 & 0.6 & - & 0.7 & 1.3 & 0.8 \\
4 & 2.3 & - & 2.4 & 4.6 & 3.8 \\
6 & 3.7 & - & 3.7 & 5.8 & 5.1 \\
\hline \hline
\end{tabular}

compared to NLO or a $-4 \%(<1 \%)$ correction compared to LO for $\mu_{r}^{2}=\mu_{f}^{2}=Q^{2}$ and $\alpha_{s} / \pi \simeq 0.1$. Altogether, we find $\sim-15 \%$ reduction of the LO prediction for the lowest partial wave and an increase of about $7 \%$ and $33 \%$ for the second and fourth, respectively. The main part of the NNLO correction arises from the $\beta_{0}$-proportional term. Its absorption in the running coupling via the BLM scale-setting prescription again requires knowledge of the nonperturbative behavior of $\alpha_{s}$. Table VI shows that then a sign change occurs at NNLO, where the BLM scale for the second partial wave is quite low and its remaining NNLO correction is rather large.

Certainly, the resummation of the $\beta$-proportional corrections is associated with a new input that is not well known. Thus, in the following discussion concerning the extraction of nonperturbative conformal moments of the distribution amplitude we prefer the naive scale-setting prescription $\mu_{r}^{2}$ $=\mu_{f}^{2}=Q^{2}$. In panels (a) and (b) of Fig. 2 we display the $\omega$ dependence for the scaled photon-to-pion transition form factor evaluated in the $\overline{\mathrm{CS}}$ scheme at LO and NNLO, respectively. One clearly sees that the prediction is almost independent of $\omega$ for $|\omega| \leqslant 0.2$ and only a negligible dependence arises for $0.2<|\omega|<0.4$. Radiative corrections will only shift this prediction downward. Note that this shift will slightly increase if we go to higher orders of $\alpha_{s}$. For the lowest partial wave they can be taken from the calculation of the radiative corrections to the Bjorken sum rule, which are evaluated in the third-loop approximation [72] and roughly estimated at four loops [73]. Consequently, confronting these predictions with experimental measurements would provide either a novel test of perturbative QCD or an insight into the size of higher-twist contributions. To enhance statistics, one can even integrate over the small $|\omega|$ region:

TABLE VI. Analogous to Table IV for the ratio $a_{\mathrm{BLM}}$ $=\mu_{\mathrm{BLM}}^{2} / Q^{2}$, where $\mu_{f}^{2}=Q^{2}$ and $|\omega|=0.8$.

\begin{tabular}{lccccc}
\hline \hline$j$ & $a_{\mathrm{BLM}}^{\overline{\mathrm{MS}}}$ & $a_{\mathrm{BLM}}^{\mathrm{CS}}$ & $a_{\mathrm{BLM}}^{\overline{\mathrm{CS}}}$ & $\frac{T_{j}^{(2)}}{2 T_{j}^{(1)}}$ & $\frac{T_{j}^{\mathrm{CS}(2)}}{2 T_{j}^{\mathrm{CS}(1)}}=\frac{T_{j}^{\overline{\mathrm{CS}}(2)}}{2 T_{j}^{\overline{\mathrm{CS}}(1)}}$ \\
\hline 0 & $1 / 7.4$ & $1 / 9.7$ & $1 / 7.4$ & - & -0.92 \\
2 & $1 / 155$ & $1 / 80$ & $1 / 62$ & - & -8.54 \\
4 & $1 / 40$ & $1 / 38$ & $1 / 28$ & - & -3.63 \\
6 & $1 / 37$ & $1 / 37$ & $1 / 27$ & - & -2.3 \\
\hline \hline
\end{tabular}




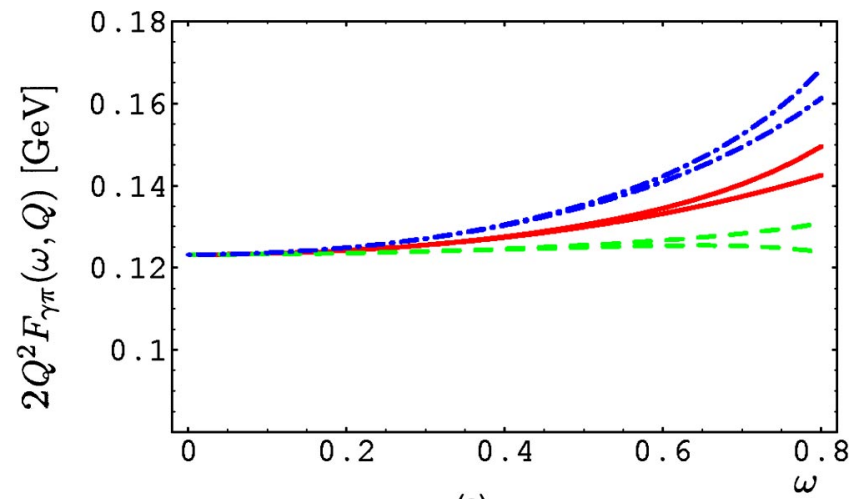

(a)

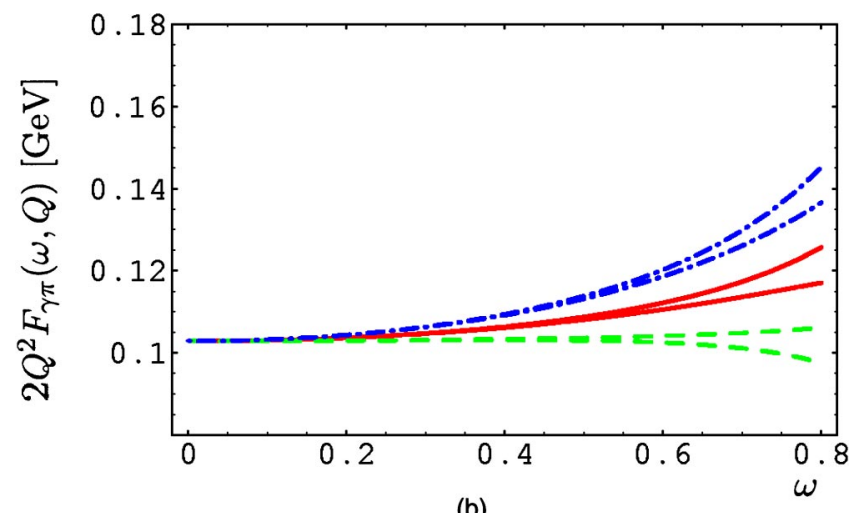

(b)

FIG. 2. The $\omega$ dependence of the scaled photon-to-pion transition form factor $2 Q^{2} F_{\gamma \pi}(\omega, Q)$ at $Q^{2}=2 \mathrm{GeV}^{2}$ in $\mathrm{LO}$ (a) and in $\overline{\mathrm{CS}}$ at NNLO (b) for three different values of $B_{2}=\{0,-0.5,0.5\}$ is shown as solid, dashed and dash-dotted lines, respectively. The spread of the corresponding lines displays the sensitivity of the predictions with respect to the parameter $B_{4}$, which is equated to -0.25 and 0.25 for the lower and upper curves, respectively.

$$
\begin{aligned}
& \frac{Q^{2}}{\omega^{\mathrm{cut}}} \int_{0}^{\omega^{\mathrm{cut}}} d \omega F_{\gamma \pi}(\omega, Q) \\
& =\frac{\sqrt{2} f_{\pi}}{3}\left\{1-\frac{\alpha_{s}(Q)}{\pi}-3.583 \frac{\alpha_{s}^{2}(Q)}{\pi^{2}}-20.215 \frac{\alpha_{s}^{3}(Q)}{\pi^{3}}\right. \\
& \left.\quad-(\sim 200) \frac{\alpha_{s}^{4}\left(Q^{2}\right)}{\pi^{4}}+O\left(\alpha_{s}^{5}\right)+O\left(m_{\pi^{2}}^{2} / Q^{2}, \Lambda^{2} / Q^{2}\right)\right\},
\end{aligned}
$$

where $\omega^{\text {cut }}<0.4$ and $n_{f}=3$.

If we vary the unknown $B_{2}$ and $B_{4}$ parameters in the range that is suggested by nonperturbative estimates, i.e., $-1 / 2 \leqslant B_{2} \leqslant 1 / 2$ and $-1 / 4 \leqslant B_{4} \leqslant 1 / 4$, it can be realized that in the intermediate $|\omega|$ region the perturbative QCD predictions start to be dependent on the value of $B_{2}$ and for larger $|\omega|$ even on that of $B_{4}$, while higher partial waves can be safely neglected. Here it is important that radiative corrections do not spoil a possible extraction. They rather shift the curves in the whole region and slightly enhance the spread of the curves associated with $B_{4}$. This is caused by the fact that the radiative corrections to the lowest two partial waves are negative and approximately of the same size, while they are positive in all other cases. Since the gap between the curves for both extreme cases, where $B_{2}= \pm 0.5$, is about $30 \%$ or even larger, we might expect that these curves can be distinguished by a measurement. This would also test the reliability of nonperturbative methods. Certainly, a precise extraction of $B_{2}$ or even $B_{4}$ also requires high-precision measurements with high statistics. Assuming that such data are available, the extraction of the nonperturbative parameter itself is rather simple. First, a measurement in the small $|\omega|$ region should be confronted with the parameter-free prediction that depends only on the running of the coupling. As we argued, we do not expect higher-twist corrections to be essential. If that were experimentally established, a simple two-parameter-dependent fit

$$
\begin{aligned}
F_{\gamma \pi}(\omega, Q)= & f_{\pi}\left[T_{0}(\omega, Q)+\frac{18}{7} T_{2}(\omega, Q) B_{2}(Q)\right. \\
& \left.+\frac{45}{11} T_{4}(\omega, Q) B_{4}(Q)\right]
\end{aligned}
$$

could be used to extract the two conformal moments $B_{2}(Q)$ and $B_{4}(Q)$ as long as the statistics is high enough. Moreover, a consistency check is provided by the $Q$ dependence of these parameters. Note that in the $\overline{\mathrm{CS}}$ scheme the mixing between different partial waves is caused by evolution at NNLO, while in the $\overline{\mathrm{MS}}$ scheme the mixing appears already at NLO in both the hard-scattering amplitude and the evolution of $B_{i}$. In the CS scheme the mixing appears first in the evolution to NLO accuracy.

Finally, we want to comment on the size of evolution effects which are caused by the off-diagonal entries in the anomalous dimension matrix. We numerically observe that the conformal symmetry breaking terms at NLO [compare the $\overline{\mathrm{MS}}$ scheme results with the CS ones in Table I, as well as at NNLO (see Table III)] can provide an enhancement of the corresponding corrections up to $50 \%$. One would naively expect a similar relative effect from the evolution due to the unknown mixing in the $\overline{\mathrm{CS}}$ scheme arising at NNLO. However, since it does not appear at the input scale $Q_{0}$ due to the initial condition (2.42), this mixing effect is in fact small [40]. For instance, at NLO in the $\overline{\mathrm{MS}}$ and CS schemes this mixing effect in the lowest partial wave goes up to $-1.3 \%$ and $2.3 \%$, respectively, for the evolution from $Q_{0}$ $=0.5 \mathrm{GeV}$ to $Q=20 \mathrm{GeV}$ and $|\omega|=1$. Note, however, that in the $\overline{\mathrm{MS}}$ scheme cancellation appears in the off-diagonal terms between the $C_{F}^{2}$ - and $C_{F} \beta_{0}$-proportional parts and that the contribution from only the $C_{F}^{2}$-proportional term might be of the order of $2 \%$. This number should be compared with the correction in the hard-scattering induced by the offdiagonal terms, which is about $7 \%$. As we discussed above, these corrections are reduced for $|\omega|<1$. Since the ratio of diagonal entries in NNLO to those in NLO is smaller than $3 \alpha_{s} / \pi$ for the first five even diagonal terms, we might argue that the ratio of off-diagonal entries in the $\overline{\mathrm{MS}}$ scheme is of the same size. Assuming so, one would expect that unknown 
mixing effects induced by $\Delta_{j k}^{\overline{\mathrm{CS}}(1)}$ due to evolution are reduced by a factor of $1 / 3$ or even more with respect to those at NLO. Thus, we expect from the numbers given above at NLO that the contribution of $\Delta_{j k}^{\overline{\mathrm{CS}}(1)}$ is smaller than $0.5 \%$ in the $\overline{\mathrm{MS}}$ scheme. In the $\overline{\mathrm{CS}}$ scheme, the contribution of the $\log$ term in Eq. (2.53) can be estimated by comparison with the NLO effect in the CS scheme. Here one finds a relative contribution smaller than $\alpha_{s}(Q) \ln \left[Q / Q_{0}\right] / \pi$. For the example discussed above that would produce a mixing effect smaller than $1 \%$. Therefore, for higher values of the input scale, e.g., $\underline{Q_{0}} \sim 1 \mathrm{GeV}$, we expect a rather tiny mixing in the $\overline{\mathrm{CS}}$ scheme.

\section{CONCLUSIONS}

We have employed conformal symmetry in the perturbative sector to evaluate the NNLO corrections for pion production through two-photon fusion. The requirement of a manifestly conformal invariant result partly removes the ambiguities arising from the factorization. However, the ambiguities are retained in the scheme dependence of the forward Wilson coefficients and anomalous dimensions and in the treatment of the conformal symmetry breaking induced by the trace anomaly, proportional to the $\beta$ function. The latter ambiguity has been studied here in two alternative schemes: (i) combining the conformal predictions with the $\overline{\mathrm{MS}}$ result and (ii) improving the partial wave decomposition of the conformal invariant theory by the renormalization group equation. The second possibility minimizes the mixing of partial waves and gives us an almost good quantum number, namely, the conformal spin. For decreasing values of $|\omega|$, the differences between these schemes are removed, since offdiagonal terms are suppressed by powers of $\omega^{2}$.

As was known before, for $|\omega|=1$ NLO corrections can be considered to be small for only the two lowest partial waves, since the NLO corrections logarithmically increase with the conformal spin. This behavior is analogous to the large $j$ behavior of the Wilson coefficients in DIS arising from soft gluon configurations. The effect is manifested by $\ln (1-x)$ terms that are associated with factorization logs and, consequently, are absent in the lowest partial wave in the conformal schemes. Other $\ln (1-x)$ terms are related to the Sudakov effect and are manifested in a sign alternating series for $\beta$ $=0$. However, the numerical study showed that the NNLO corrections are dominated by the $\beta_{0}$-proportional term, as expected. In general, this term is rather large compared with the NLO coefficient and thus the BLM scale is rather low, which drives the coupling in the nonperturbative region.

We compared the NNLO predictions with the existing data for the quasireal photon case and found that for $2 Q^{2}$ $>2 \mathrm{GeV}^{2}$ the contribution from the lowest partial wave is compatible with the data. The deviation below this scale is induced by nonperturbative effects or by the contribution of higher partial waves and it requires a deeper insight into power suppressed contributions. Although there is no doubt in the literature that the CLEO measurement can be analyzed in this limit, we should state here that partial waves with sufficiently large conformal spin will be exponentially sup- pressed. This can affect the analysis only if the matrix elements of conformal operators with rather large conformal spin contribute. On the other hand, we know that the net effect of all partial waves with conformal $\operatorname{spin} j+1 \geqslant 3$ is small. Obviously, this does not necessarily mean that the matrix elements themselves are small, and therefore we cannot say that the asymptotic form of the pion distribution amplitude is experimentally established. Also, taking into account nonperturbative results from other methods given in the literature, a strong statement that the asymptotic form is suggested by these estimates cannot in fact be made.

These problems that we have spelled out can be separately studied apart from the $|\omega| \rightarrow 1$ limit. Indeed, in the small $|\omega|$ region, perturbative QCD predicts a sum rule that has the same status as the Bjorken sum rule in deep inelastic scattering, evaluated at order $\alpha_{s}^{3}$. A first test of this sum rule might be possible with existing $e^{+} e^{-}$machines and would offer us a first insight into the size of power suppressed contributions for exclusive processes from experimental data. We expect that such contributions will turn out to be small. If this should be established experimentally, one might attack the extraction of the first- and second-lowest conformal moments of the distribution amplitude. This is an important task, since it would open a window to testing the reliability of nonperturbative methods applied to exclusive quantities. Having in mind that the collinear factorization applied here to the photon-to-pion transition form factor is also adopted for the analysis of exclusive $B$ physics, it is timely to confront such methods with experimental measurements.

Let us finally give a short outlook for the application of the conformal approach to other processes. After a simple replacement of the decay constant and matrix elements the NNLO result obtained can be used for the analysis of $\eta$ production, i.e., its flavor octet component. Moreover, the formalism can be extended in a straightforward manner to the $\eta^{\prime}$-to-photon transition form factor. Guided by the large $j+1$ asymptotics of the conformal moments, it is also possible to reconstruct the hard-scattering amplitude in DVCS. The reliability of this technique can be tested at NLO and partly also at NNLO, i.e., for $\beta$-proportional terms. We also want to add that one can go one order further in $\alpha_{s}$ in the approximation of the first few conformal moments of the hard-scattering amplitude, since we can borrow the forward Wilson coefficients from the nonsinglet sector of the deep inelastic structure function $F_{3}$, evaluated at next-to-NNLO [58].

\section{ACKNOWLEDGMENTS}

We would like to thank M. Diehl and P. Kroll for useful discussions. One of us (B.M.) acknowledges support by the Alexander von Humboldt Foundation. This work was partially supported by the Ministry of Science and Technology of the Republic of Croatia under Contract No. 0098002.

\section{APPENDIX A: STRUCTURE OF THE HARD-SCATTERING AMPLITUDE IN THE $\overline{M S}$ SCHEME}

Here we prove that the $\mathrm{LN}(\omega, x)$ terms, appearing in Eqs. (3.18)-(3.21), are related to the factorization logs. We do not 
distinguish between the renormalization and the factorization scales, since the difference appears only in the $\omega$-independent term $\ln \left(\mu_{r} / \mu_{f}\right)$. The hard-scattering amplitude is given by the sum over all Feynman diagram $\mathcal{F}$ contributions

$$
\frac{1}{Q^{2}} T(\omega, x, Q / \mu)=\sum_{\mathcal{F}} T_{\mathcal{F}}(\omega, x, Q / \mu),
$$

where we have rescaled the individual contributions by $Q^{2}$ to have dimensionless amplitudes. Each of these contributions is given as a product of propagators and vertices integrated over the virtual loop momenta. The two photon vertices are connected by a chain of quark propagators $S$ and quarkgluon-quark vertices $V$ :

$$
\begin{aligned}
& \$([1-2 x] P / 2+q-l) V_{1} \$\left([1-2 x] P / 2+q-l+k_{1}\right) \\
& \times \cdots \$\left([1-2 x] P / 2+q-l+\cdots+k_{n}\right),
\end{aligned}
$$

where $P=q_{1}+q_{2}$ and the large momentum $q=\left(q_{1}-q_{2}\right) / 2$ flows only into this chain. Momentum conservation requires that $l+l^{\prime}=\sum_{i=1}^{n} k_{i}$, where $l$ and $l^{\prime}$ is the sum of virtual momenta flowing into the first and flowing out of the second photon vertex, respectively. Interchanging the two photon vertices will give the crossed contributions with $|\omega| \rightarrow-\omega$. Obviously, there are further propagators that depend only on the virtual momenta and $x P$ or $(1-x) P$, but not on $q$. Introducing the Feynman-Schwinger representation for the propagators, integrating over the virtual momenta, and making use of the on-shell condition $P^{2}=0$, give us the following representation for the regularized contribution:

$$
\begin{aligned}
T_{\mathcal{F}}(\omega, x, Q / \mu)= & \int_{0}^{1} d z_{1} \int_{0}^{z_{1}} d z_{2} \cdots \int_{0}^{z_{m-1}} d z_{m} \\
& \times \frac{\mu^{2 \epsilon} \mathcal{T}_{\mathcal{F}}(\underline{z} \mid \epsilon)}{Q^{2 \epsilon}\left[x \omega \mathcal{B}_{1}(\underline{z})+(1-x) \omega \mathcal{B}_{2}(\underline{z})+1\right]^{1+\epsilon}} .
\end{aligned}
$$

Here $\epsilon$ is the dimensional regularization parameter and the functions $\mathcal{B}_{i}(z)$ depend on the Feynman-Schwinger variables $\underline{z}=\left\{z_{1}, \ldots, \overline{z_{m}}\right\}$ with $n \leqslant m$. We introduce the new variable

$$
y=\frac{1}{2}-\frac{x}{2} \mathcal{B}_{1}(\underline{z})-\frac{1-x}{2} \mathcal{B}_{2}(\underline{z})
$$

and write

$$
T_{\mathcal{F}}(\omega, x, Q / \mu)=\int_{0}^{1} d y \frac{\mu^{2 \epsilon}}{Q^{2 \epsilon}[1-(2 y-1) \omega]^{1+\epsilon}} V_{\mathcal{F}}(y, x \mid \epsilon),
$$

where the unrenormalized convolution kernel is defined by

$$
\begin{aligned}
V_{\mathcal{F}}(y, x \mid \epsilon)= & \int_{0}^{1} d z_{1} \int_{0}^{z_{1}} d z_{2} \cdots \int_{0}^{z_{m-1}} d z_{m} \delta\left(y-\frac{1}{2}+\frac{x}{2} \mathcal{B}_{1}(\underline{z})\right. \\
& \left.+\frac{1-x}{2} \mathcal{B}_{2}(\underline{z})\right) \mathcal{T}_{\mathcal{F}}(\underline{z} \mid \epsilon) .
\end{aligned}
$$

The renormalization procedure provides the factorization $\operatorname{logs} \ln \left(Q^{2} / \mu^{2}\right)$, which always appear in combination with $\mathrm{LN}(\omega, x)$ terms. The factorization theorem tells us that after resummation of all Feynman diagrams the corresponding convolution kernel is given by the evolution one. Obviously, the log-independent terms can also be represented as convolution. The support of all these kernels is known and follows from the restrictions on $\mathcal{B}_{i}(z)$, which are obtained from their definition and the topology of Feynman graphs (see, for instance, $[74,4])$.

\section{APPENDIX B: CONSISTENCY CHECK WITH THE FORWARD-LIMIT RESULTS}

In this section we present a consistency check between the results for the nonsinglet coefficient function of the DIS polarized structure function $g_{1}$ [43] and the hard-scattering amplitude of the pion transition form factor $[9,10]$. The former quantity is known to NNLO, while for the latter discussed in Sec. III A the calculation has been performed up to $\beta_{0}$-proportional NNLO terms. Both results have been obtained in the $\overline{\mathrm{MS}}$ scheme. Making use of the fact that both quantities, the photon-to-pion transition form factor and the structure function $g_{1}$, are defined by the two-photon amplitudes belonging to a general class of the scattering amplitudes for the two-photon process at lightlike distances, we are able to transform the results for the photon-to-pion transition form factor to the results for $g_{1}$.

The general scattering amplitude for the two-photon processes is given by the time-ordered product of two electromagnetic currents sandwiched between the in and out hadronic states with momenta $P_{1}$ and $P_{2}$, respectively. Using the notation $q=\left(q_{1}+q_{2}\right) / 2\left(q_{1}\right.$ and $q_{2}$ are incoming and outgoing photon momenta), $P=P_{1}+P_{2}$, and $\Delta=P_{2}-P_{1}$, the following generalized Bjorken region can be defined [4,39]:

$$
\nu=P \cdot q \rightarrow \infty \text { and } Q^{2}=-q^{2} \rightarrow \infty,
$$

with the scaling variables

$$
\omega=\frac{\nu}{Q^{2}} \quad \text { and } \quad \eta=\frac{\Delta \cdot q}{\nu} .
$$

In the forward case, corresponding to DIS, $1 / \omega$ can be identified with the Bjorken variable $x_{B j}$ and $\eta$ vanishes, while for the two-photon production of a hadron $\eta=1$. The relations between the nonforward ERBL kernels and the forward DGLAP kernels were extensively studied and derived in Ref. [4], while in Ref. [39] consistency between the transition form factor and $g_{1}$ results was reported up to NLO. Here we explain in more detail the technical side of these consistency checks and extend them to $\beta_{0}$-proportional NNLO terms. 
The building blocks of the hard-scattering amplitude for the photon-to-pion transition form factor $T\left(\omega, x, Q, \mu_{f}\right)$ given in Eqs. (1.2), (1.3), and (3.16)-(3.23) can generally be written as

$$
\begin{aligned}
& \mathcal{A}_{1}^{\left(\gamma^{*} \pi\right)}(\omega, x)=\frac{1}{1-\omega(2 y-1)-i \varepsilon} \otimes \delta(y-x), \\
& \mathcal{A}_{2, n}^{\left(\gamma^{*} \pi\right)}(\omega, x)=\frac{\ln ^{n}[1-\omega(2 y-1)-i \varepsilon]}{1-\omega(2 y-1)-i \varepsilon} \otimes[\tilde{v}(y, x)]_{+},
\end{aligned}
$$

where $n=0,1,2$. Note that we have reintroduced the $i \varepsilon$ term, originally present in the definition of Feynman propagators. For the kernels that appear in Eqs. (3.16)-(3.23) we use the generic symbol $\tilde{v}(y, x)$. Furthermore, for the kernels of interest, given in Eqs. (3.7), (3.8), and (3.11), the function $\tilde{v}$ is of the general form

$$
\tilde{v}(x, y)=\theta(y-x) \tilde{f}(x, y)+\left\{\begin{array}{l}
x \rightarrow \bar{x} \\
y \rightarrow \bar{y}
\end{array}\right\} .
$$

We have to extend our restricted nonforward kinematics to the whole kinematical region. The extension of the $\mathrm{BL}$ kernels $\tilde{v}$ to the whole $x, y$ region $(-\infty<x, y<\infty)$ [4] is accompanied by a change of the $\theta$ function as follows:

$$
\tilde{v}^{\operatorname{ext}}(x, y)=\tilde{v}(x, y)_{\mid \theta(y-x) \rightarrow \theta(1-x / y) \theta(x / y) \operatorname{sgn}(y)} .
$$

Furthermore, the dependence on $\eta$ has to be restored and one performs the following change of variables:

$$
x \rightarrow \frac{1+t / \eta}{2}, \quad y \rightarrow \frac{1+t^{\prime} / \eta}{2}, \quad \omega \rightarrow \eta \omega .
$$

The definition of the distribution amplitudes, with which the hard-scattering amplitude is convoluted, as well as the definition of its generalized counterpart, introduce the restriction $-1<t<1$. After examining the $\theta$ functions in the kernels (B5) and taking into account $|\eta| \leqslant 1$, one obtains $-1<t^{\prime}$ $<1$. The building blocks of the generalized two-photon scattering amplitude are thus obtained and they are of the form

$$
\begin{aligned}
\mathcal{A}_{1}(\omega, \eta, t)= & \frac{1}{1-\omega t-i \varepsilon}, \\
\mathcal{A}_{2, n}(\omega, \eta, t)= & \int_{-1}^{1} d t^{\prime} \quad \frac{\ln ^{n}\left(1-\omega t^{\prime}-i \varepsilon\right)}{1-\omega t^{\prime}-i \varepsilon} \\
& \times \frac{1}{2 \eta}\left[\tilde{v}^{\operatorname{ext}}\left(\frac{1+t^{\prime} / \eta}{2}, \frac{1+t / \eta}{2}\right)\right]_{+} .
\end{aligned}
$$

It is easy to see that relations (B3) indeed represent the $\eta$ $=1$ limit of (B7).

The forward limit (i.e., the forward Compton amplitude) corresponds to $\eta \rightarrow 0$ and, due to the optical theorem, the nonsinglet coefficient functions of the DIS polarized structure function $g_{1}$ (contributing to the total cross section) are determined by taking the imaginary part of the forward am- plitude. Taking into account the definition of $g_{1}$ and its coefficient functions (see [43]), one arrives at the following recipe $^{6}$ for the building blocks of the nonsinglet coefficient functions $C_{q}^{\mathrm{NS}}$ :

$$
\mathcal{A}_{i}^{\left(g_{1}\right)}(z)=\frac{\omega}{\pi} \operatorname{Im}\left[\lim _{\eta \rightarrow 0} \mathcal{A}_{i}(\omega, \eta, t)\right]_{\mid \omega=1 / z, t=1} .
$$

The $\eta \rightarrow 0$ limit of the extended ERBL kernels [4] results in the corresponding DGLAP kernels $\widetilde{P}$ of the general form

$$
\begin{aligned}
\widetilde{P}(z) & =\theta(z) \theta(1-z) \tilde{p}(z) \quad \text { with } \\
{[\widetilde{P}(z)]_{+} } & =\widetilde{P}(z)-\delta(1-z) \int_{0}^{1} d z^{\prime} \widetilde{P}\left(z^{\prime}\right) .
\end{aligned}
$$

It is straightforward to derive

$$
\lim _{\eta \rightarrow 0} \frac{1}{2 \eta}\left[\tilde{v} \operatorname{ext}\left(\frac{1+t^{\prime} / \eta}{2}, \frac{1+t / \eta}{2}\right)\right]_{+}=\operatorname{sgn}(t) \frac{1}{t}\left[\widetilde{P}\left(\frac{t^{\prime}}{t}\right)\right]_{+},
$$

with $\tilde{p}$ given by

$$
\begin{aligned}
\frac{1}{t} \tilde{p}\left(\frac{t^{\prime}}{t}\right)= & \lim _{\eta \rightarrow 0} \frac{1}{2 \eta}\left[\tilde{f}\left(\frac{1+t^{\prime} / \eta}{2}, \frac{1+t / \eta}{2}\right)\right. \\
& \left.-\widetilde{f}\left(\frac{1-t^{\prime} / \eta}{2}, \frac{1-t / \eta}{2}\right)\right]_{+} .
\end{aligned}
$$

The imaginary part of expressions (B7) is obtained by making use of

$$
\operatorname{Im} \frac{1}{1-t^{\prime} \omega-i \varepsilon}=\pi \delta\left(1-t^{\prime} \omega\right)
$$

and for more complicated functions, containing $\ln ^{n}\left(1-t^{\prime} \omega\right.$ $-i \varepsilon)(n=1,2)$, we derive the following decompositions:

$$
\begin{aligned}
\frac{\ln \left(1-\omega t^{\prime}-i \varepsilon\right)}{1-\omega t^{\prime}-i \varepsilon} & =\frac{1}{(1-s)_{+}} \otimes \frac{1}{1-s t^{\prime} \omega-i \varepsilon}, \\
\frac{\ln ^{2}\left(1-\omega t^{\prime}-i \varepsilon\right)}{1-\omega t^{\prime}-i \varepsilon}= & 2\left(\frac{\ln (1-s)-\ln s}{1-s}\right)_{+} \\
& \otimes \frac{1}{1-s t^{\prime} \omega-i \varepsilon} .
\end{aligned}
$$

Alternatively, for the imaginary parts of the expressions containing logarithms one can refer to [78]. Finally, we present the results relevant to the NNLO calculation:

\footnotetext{
${ }^{6}$ The factor $1 /(2 \pi)$ comes from the dispersion relation, and the additional factor of 2 from the definition of $g_{1}$. The origin of the factor $\omega$ in Eq. (B8) lies in the fact that the transition form factor is scaled by $Q^{2}$, while the forward Compton amplitude is scaled by $P \cdot q$.
} 
TABLE VII. Selected forward counterparts of the nonforward quantities.

\begin{tabular}{|c|c|c|}
\hline$\delta(x-y)$ & $\delta(1-z)$ & \\
\hline$v^{a}(x, y)$ & $(1-z) \theta(z) \theta(1-z)$ & \\
\hline$v^{b}(x, y)$ & $\frac{2 z}{1-z} \theta(z) \theta(1-z)$ & \\
\hline$v(x, y)$ & $\frac{1+z^{2}}{1-z} \theta(z) \theta(1-z)$ & \\
\hline$g(x, y)$ & $-\frac{2 \ln (1-z)}{1-z} \theta(z) \theta(1-z)$ & \\
\hline$\dot{v}^{a}(x, y)$ & $(1-z)(\ln z+1) \theta(z) \theta(1-z)$ & \\
\hline$\dot{v}(x, y)$ & {$\left[(1-z)+\frac{1+z^{2}}{1-z} \ln z\right] \theta(z) \theta(1-z)$} & \\
\hline$\ddot{v}(x, y)$ & {$\left[2(1-z) \ln z+\frac{1+z^{2}}{1-z} \ln ^{2} z\right] \theta(z) \theta(1-z)$} & \\
\hline$\dot{g}(x, y)$ & {$\left[-\frac{\pi^{2}}{3(1-z)}-\frac{\ln ^{2}(1-z)}{1-z} \ln z+\frac{2 \mathrm{Li}_{2}(1-z)}{1-z}\right] \theta$} & $\theta(z) \theta(1-z)$ \\
\hline$\frac{\ln [1-\omega(2 y-1)]}{1-\omega(2 y-1)} \otimes v(y, x) \rightarrow$ & $\left\{-(1-z)+\frac{3}{2(1-z)}+\frac{2}{1-z} \ln (1-z)+\frac{1+z^{2}}{1-z}[\ln (1-z)-\ln z]\right.$ & ]$\theta(z) \theta(1-z)$ \\
\hline
\end{tabular}

$$
\begin{aligned}
\mathcal{A}_{1}^{\left(g_{1}, \mathrm{NS}\right)}(z) & =\delta(1-z) \\
\mathcal{A}_{2,0}^{\left(g_{1}, \mathrm{NS}\right)}(z)= & {[\widetilde{P}(z)]_{+} } \\
\mathcal{A}_{2,1}^{\left(g_{1}, \mathrm{NS}\right)}(z)= & \theta\left(\frac{z}{z^{\prime}}\right) \theta\left(1-\frac{z}{z^{\prime}}\right) \frac{1}{\left(z^{\prime}-z\right)_{+}} \otimes\left[\widetilde{P}\left(z^{\prime}\right)\right]_{+} \\
\mathcal{A}_{2,2}^{\left(g_{1}, \mathrm{NS}\right)}(z)= & \theta\left(\frac{z}{z^{\prime}}\right) \theta\left(1-\frac{z}{z^{\prime}}\right) \\
& \times 2\left(\frac{\ln \left(z^{\prime}-z\right)-\ln z}{z^{\prime}-z}\right)_{+} \otimes\left[\widetilde{P}\left(z^{\prime}\right)\right]_{+}
\end{aligned}
$$

Hence, the building blocks for the hard-scattering amplitude of the photon-to-pion transition form factor $\mathcal{A}_{i}^{\left(\gamma^{*} \pi\right)}(\omega, x)$ given in Eq. (B3) can be brought into correspondence with the building blocks of the nonsinglet coefficient function of the polarized structure function $g_{1}$, $\mathcal{A}_{i}^{\left(g_{1}, \mathrm{NS}\right)}(z)$, displayed in Eq. (B14). For various ERBL kernels $\tilde{v}(x, y)$, the corresponding DGLAP kernels $\widetilde{P}(z)$ are obtained by taking the limit (B11) with $t=1 \quad$ (and $t^{\prime} \rightarrow z$ ) taken into account. In Table VII we list some selected results. We mention here that the integration of two "+" forms results again in the "+" form, but the contributing terms should be appropriately rearranged. Following the procedure explained above, we finally obtain the forward counterparts of the elements of the hard-scattering amplitude for the photon-to-pion transition form factor:

$$
\begin{aligned}
& T^{(0)}(\omega, x) \rightarrow \delta(1-z), \\
& T_{F}^{(1)}(\omega, x) \rightarrow\left[-(1-z)+\frac{3}{2(1-z)}-\frac{3 z}{(1-z)}+\frac{1+z^{2}}{1-z}[\ln (1-z)-\ln z]\right]_{+}-\frac{3}{2} \delta(1-z),
\end{aligned}
$$




$$
\begin{aligned}
T_{\beta}^{(2)}(\omega, x) \rightarrow & {\left[-\frac{31}{12}(1-z)+\frac{19}{4(1-z)}-\frac{209\left(1+z^{2}\right)}{36(1-z)}+\left(\frac{3}{2}(1-z)-\frac{3}{2(1-z)}+\frac{19\left(1+z^{2}\right)}{6(1-z)}\right) \ln (1-z)+\left(-\frac{1}{4}(1-z)+\frac{5}{2} z\right.\right.} \\
& \left.\left.-\frac{19\left(1+z^{2}\right)}{4(1-z)}\right) \ln (z)+\frac{1+z^{2}}{1-z}\left(-\frac{5}{4} \ln ^{2} z-\frac{1}{2} \ln ^{2}(1-z)+2 \ln z \ln (1-z)+\operatorname{Li}_{2}(1-z)+\frac{\pi^{2}}{3}\right)\right]_{+}-3 \delta(1-z) .
\end{aligned}
$$

Here, the expressions on the RHS represent the scaleindependent LO, NLO, and $\beta_{0}$-proportional NNLO terms of the nonsinglet coefficient function $\left(C_{q}^{\mathrm{NS}}\right)$ of $g_{1}$. Similar expressions can be written for the terms proportional to $\ln ^{n}\left(Q^{2} / \mu^{2}\right)$. Following the notation of [43], the $\theta(z) \theta(1-z)$ factors are not shown in Eqs. (B15)-(B17). We note that the limit of $T^{(i)}(\omega, 1-x)=T^{(i)}(-\omega, x)$ corresponds to the antiquark case $\left(C_{\bar{q}}^{\mathrm{NS}}\right)$.

Our results $^{7}$ (B15)-(B17) are numerically in agreement with the Mellin moments and up to a typo also with the analytical expression for the $n_{f}$-proportional term displayed in the Appendix of Ref. [43]; namely, in Eq. (A2) in that reference the term $1 / 3(1+11 z) \ln z$ should read $1 / 3(1$ $-11 z) \ln z$.

\section{APPENDIX C: DETERMINATION OF CONFORMAL MOMENTS}

In this section we present a method for computing moments with respect to conformal partial waves with the index $k$. We introduce the notation

$$
\langle F(x)\rangle_{k} \equiv \int_{0}^{1} d x F(x) \frac{x(1-x)}{N_{k}} C_{k}^{3 / 2}(2 x-1),
$$

while $N_{k}$ is defined in Eq. (2.1). It follows trivially that $\langle F(1-x)\rangle_{k}=(-1)^{k}\langle F(x)\rangle_{k}$. In the calculation of the photon-to-pion transition form factor for the special case $|\omega|=1$ we encounter functions $F(x)$ of the forms $f(x) / x$ and $f(x) /(1-x), \quad$ with $f(x) \in\left\{1, \quad \ln ^{n}(x) \ln ^{m}(1-x), \quad \operatorname{Li}_{2}(x)\right.$, $\left.\mathrm{Li}_{3}(x), \mathrm{S}_{12}(x)\right\}$.

It is convenient to use the following expression for the Gegenbauer polynomials:

$$
\frac{x(1-x)}{N_{k}} C_{k}^{3 / 2}(2 x-1)=(-1)^{k} \frac{2(2 k+3)}{(k+1) !} \frac{d^{k}}{d x^{k}}[x(1-x)]^{k+1}
$$

${ }^{7}$ The representation of the coefficient functions in the form $A \delta(1-z)+[F(z)]_{+}$, as given in Eqs. (B15)-(B17) and naturally emerging in our calculation, is convenient for the determination of the Mellin moments $c_{j}=\int_{0}^{1} z^{j} c(z)$ since the $j=0$ Mellin moment corresponds to the term proportional to $\delta(1-z)$.

$$
\begin{aligned}
= & (-1)^{k} \frac{2(2 k+3)}{(k+1)} \sum_{i=0}^{k+1}(-1)^{i}\left(\begin{array}{c}
k+1 \\
i
\end{array}\right) \\
& \times\left(\begin{array}{c}
k+i+1 \\
i+1
\end{array}\right) x^{i+1} .
\end{aligned}
$$

The evaluation of the conformal moments, i.e., in our case the evaluation of the expressions

$$
\begin{aligned}
\left\langle\frac{f(x)}{x}\right\rangle_{k}= & (-1)^{k} \frac{2(2 k+3)}{(k+1)} \sum_{i=0}^{k+1}(-1)^{i}\left(\begin{array}{c}
k+1 \\
i
\end{array}\right) \\
& \times\left(\begin{array}{c}
k+i+1 \\
i+1
\end{array}\right) \int_{0}^{1} x^{i} f(x),
\end{aligned}
$$

and

$$
\begin{aligned}
\left\langle\frac{f(x)}{1-x}\right\rangle_{k}= & \frac{2(2 k+3)}{(k+1)} \sum_{i=0}^{k+1}(-1)^{i}\left(\begin{array}{c}
k+1 \\
i
\end{array}\right)\left(\begin{array}{c}
k+i+1 \\
i+1
\end{array}\right) \\
& \times \int_{0}^{1} x^{i} f(1-x),
\end{aligned}
$$

consists then in calculating the Mellin moments and performing the summation. The Mellin moments for the functions we encounter in our calculation are well known (see, for example, $[75,76]$ ), and most of the nontrivial sums we are left with can be found in [77]. The sums that usually appear are

$$
\mathrm{S}_{m}(n)=\sum_{i=1}^{n} \frac{1}{i^{m}}, \quad \mathrm{~S}_{m, j_{1}}, \ldots, j_{p}(n)=\sum_{i=1}^{n} \frac{1}{i^{m}} \mathrm{~S}_{j_{1}}, \ldots, j_{p}(i),
$$

$$
\begin{gathered}
\mathrm{S}_{-m}(n)=\sum_{i=1}^{n} \frac{(-1)^{i}}{i^{m}} \\
\mathrm{~S}_{-m, j_{1}, \ldots, j_{p}}(n)=\sum_{i=1}^{n} \frac{(-1)^{i}}{i^{m}} \mathrm{~S}_{j_{1}, \ldots, j_{p}}(i) .
\end{gathered}
$$

The functions $S_{m}(z)$ are expressed via the functions $\psi(z)$ $=d \ln \Gamma(z) / d z$ :

$$
\psi(z)=-\gamma_{\mathrm{E}}+S_{1}(z-1),
$$




$$
\frac{d^{m}}{d z^{m}} \psi(z)=m !(-1)^{(m+1)}\left[\zeta(m+1)-S_{m+1}(z-1)\right]
$$

For $\left\langle\operatorname{Li}_{3}(x) /(1-x)\right\rangle_{k}$ and $\left\langle\mathrm{S}_{12}(x) /(1-x)\right\rangle_{k}$, the corresponding sums are missing in [77], and to obtain them we turn to expressing the relevant functions as convolutions of appropriate functions with the known diagonal kernels. Generally, the conformal moments of a kernel $[\tilde{v}]_{+}$are defined by

$$
\begin{aligned}
\tilde{v}_{l k} \equiv & \left\langle[\tilde{v}(x, y)]_{+}\right\rangle_{l k} \\
= & \int_{0}^{1} d x \int_{0}^{1} d y C_{l}^{3 / 2}(2 x-1)[\tilde{v}(x, y)]_{+} \frac{y(1-y)}{N_{k}} \\
& \times C_{k}^{3 / 2}(2 y-1),
\end{aligned}
$$

and for the kernels appearing in this calculation $\tilde{v}_{l k}=0$ for $l<k$ and $l-k$ odd. The conformal moments of the convolution $F(x)=G(y) \otimes[\tilde{v}(y, x)]_{+}$then take the form

$$
\langle F(x)\rangle_{k}=\left\langle G(y) \otimes[\tilde{v}(y, x)]_{+}\right\rangle_{k}=\sum_{l \geqslant k}\langle G(y)\rangle_{l} \tilde{v}_{l k} .
$$

As before we use the simplified notation for the diagonal moments

$$
\tilde{v}_{k k} \equiv \tilde{v}_{k}
$$

i.e., for the diagonal conformal moments we retain just one index and the relation (C8) simplifies to

$$
\langle F(x)\rangle_{k}=\left\langle G(y) \otimes[\tilde{v}(y, x)]_{+}\right\rangle_{k}=\langle G(y)\rangle_{k} \tilde{v}_{k} .
$$

Hence, in order to determine $\left\langle\mathrm{Li}_{3}(x) /(1-x)\right\rangle_{k}$ and $\left\langle\mathrm{S}_{12}(x) /(1-x)\right\rangle_{k}$, we make use of the identities

$$
\begin{aligned}
& \frac{\mathrm{Li}_{2}(1-y)}{1-y} \otimes\left[v^{a}(y, x)\right]_{+} \\
& =-\frac{\mathrm{Li}_{3}(1-x)}{x}+\frac{\zeta(3)}{x}-\frac{\ln (x)}{1-x}+\frac{\ln (x) \ln (1-x)}{2(1-x)} \\
& \quad+\frac{1}{2}\left(\frac{\mathrm{Li}_{2}(x)}{1-x}-\frac{\zeta(2)}{1-x}\right)+\left(\frac{\mathrm{Li}_{2}(1-x)}{x}-\frac{\zeta(2)}{x}\right)
\end{aligned}
$$

and

$$
\begin{aligned}
\frac{\mathrm{Li}_{2}(y)}{1-y} \otimes[v(y, x)]_{+} \\
=-\frac{\mathrm{S}_{12}(x)}{1-x}+\frac{\zeta(3)}{1-x}+\frac{\ln (1-x)}{x}+\frac{\mathrm{Li}_{2}(x)}{2(1-x)} \\
\quad+\zeta(2)\left(\frac{\ln (1-x)}{1-x}+\frac{1}{1-x}\right)
\end{aligned}
$$

The kernels $v^{a}$ and $v$ are defined in Eqs. (3.2), (3.3), and (3.9), while the corresponding moments can be read off from Eqs. (3.6) (for $\epsilon=0$ ) and (3.34), respectively.

Finally, in Table VIII we summarize the conformal moments of the functions relevant to our calculation.

As a by-product of this calculation, we list the following nontrivial sums:

$$
\begin{aligned}
& \sum_{i=0}^{n}(-1)^{j}\left(\begin{array}{l}
n \\
j
\end{array}\right)\left(\begin{array}{c}
n+j \\
j+1
\end{array}\right) S_{1,2}(j+1) \\
& =\frac{1}{n+1}\left[-\frac{1}{n(n+1)}-(-1)^{n}\left[S_{-2}(n+1)\right.\right. \\
& \left.\left.+S_{-2}(n-1)\right]\right] \\
& \sum_{i=0}^{n}(-1)^{j}\left(\begin{array}{c}
n \\
j
\end{array}\right)\left(\begin{array}{c}
n+j \\
j+1
\end{array}\right) \frac{S_{1,2}(j+1)}{j+1} \\
& =\frac{1}{n+1}\left[\frac{(-1)^{n}}{n(n+1)}\left[S_{-2}(n+1)+S_{-2}(n-1)\right]\right], \\
& \sum_{i=0}^{n}(-1)^{j}\left(\begin{array}{l}
n \\
j
\end{array}\right)\left(\begin{array}{c}
n+j \\
j+1
\end{array}\right) \frac{S_{3}(j+1)}{j+1} \\
& =\frac{1}{n+1}\left[-\frac{1}{n^{2}(n+1)^{2}}+\left[S_{1}(n+1)+S_{1}(n-1)\right]\right. \\
& \left.\times\left(\frac{1}{n(n+1)}+\frac{1}{n^{2}(n+1)^{2}}\right)\right],
\end{aligned}
$$

which complement the collection of sums found in [77].

\section{APPENDIX D: TAYLOR EXPANSIONS IN $\omega$}

We now present the results for the five lowest even partial waves, which are expanded in $\omega^{2}$ to the first seven nonvanishing terms. For brevity, we will not denote the neglected terms.

The LO result can be simply expanded by means of its representation in terms of hypergeometrical functions. Employing the identity

$$
\begin{aligned}
& \frac{1}{(1+\omega)^{j+1+\epsilon}}{ }_{2} F_{1}\left(\begin{array}{c|c}
j+1+\epsilon, j+2+\epsilon & 2 \omega \\
2(j+2+\epsilon) & 1+\omega
\end{array}\right) \\
& ={ }_{2} F_{1}\left(\begin{array}{c|c}
j / 2+\epsilon / 2+1 / 2, j / 2+\epsilon / 2+1 \\
j+\epsilon+5 / 2
\end{array} \mid \omega^{2}\right)
\end{aligned}
$$

and representing the hypergeometrical functions as power series in $\omega$, after a few simple manipulation with $\Gamma$ functions we find

$$
T_{j}^{(0)}(\omega)=\frac{3+2 j}{4} \sum_{n=0}^{\infty} \frac{\sqrt{\pi} \Gamma(1+j+2 n)}{n ! \Gamma(5 / 2+j+n)}\left(\frac{\omega}{2}\right)^{2 n+j} .
$$


TABLE VIII. The conformal moments of some relevant functions.

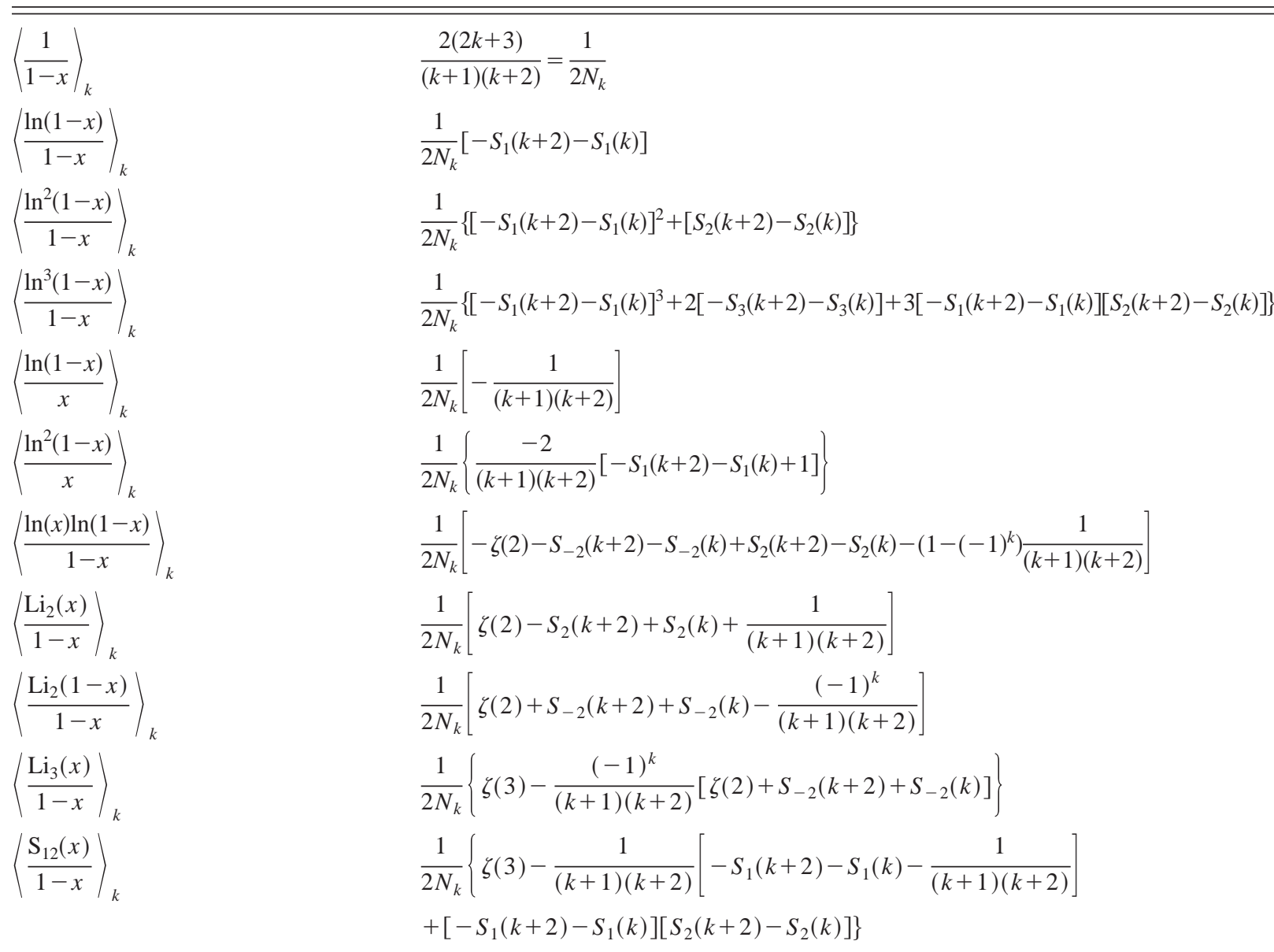

The first few moments read

$$
\begin{aligned}
T_{0}^{(0)} \simeq & +0.2 \omega^{2}+0.0857 \omega^{4}+0.0476 \omega^{6}+0.0303 \omega^{8} \\
& +0.0210 \omega^{10}+0.0154 \omega^{12} \\
T_{2}^{(0)} \simeq & \frac{2 \omega^{2}}{15}\left(1+0.6667 \omega^{2}+0.4545 \omega^{4}+0.3263 \omega^{6}\right. \\
& \left.+0.2448 \omega^{8}+0.1900 \omega^{10}+0.1517 \omega^{12}\right) \\
T_{4}^{(0)} \simeq & \frac{8 \omega^{4}}{315}\left(1+1.1538 \omega^{2}+1.0769 \omega^{4}+0.9502 \omega^{6}\right. \\
& \left.+0.8252 \omega^{8}+0.7152 \omega^{10}+0.6219 \omega^{12}\right) \\
T_{6}^{(0)} \simeq & \frac{16 \omega^{6}}{3003}\left(1+1.6471 \omega^{2}+1.9505 \omega^{4}+2.0433 \omega^{6}\right. \\
& \left.+2.0211 \omega^{8}+1.9403 \omega^{10}+1.8325 \omega^{12}\right) \\
T_{8}^{(0)} \simeq & \frac{128 \omega^{8}}{109395}\left(1+2.1429 \omega^{2}+3.0745 \omega^{4}+3.7304 \omega^{6}\right. \\
& \left.+4.1449 \omega^{8}+4.3736 \omega^{10}+4.4677 \omega^{12}\right)
\end{aligned}
$$

The relative error of these approximations for $j$ $=\{0,2,4,6,8\}$ is about $\{0.1 \%, 0.7 \%, 2 \%, 4.4 \%, 8 \%\}$ for $|\omega|$ $=0.8$ and increases to $\{0.6 \%, 4 \%, 10 \%, 19 \%, 30 \%\}$ for $|\omega|$ $=0.9$.

The expansion of the $s_{j}^{i}(\omega)$ functions from Eq. (3.63) can be found in an analogous way:

$s_{j}^{(i)}(\omega)$

$$
=\frac{\sum_{n=0}^{\infty} \mathcal{S}^{(i)}(j, n) \Gamma(1+j+2 n) /[n ! \Gamma(5 / 2+j+n)](\omega / 2)^{2 n}}{\sum_{n=0}^{\infty} \Gamma(1+j+2 n) /[n ! \Gamma(5 / 2+j+n)](\omega / 2)^{2 n}},
$$

$$
\begin{aligned}
\mathcal{S}^{(1)}(j, n)= & S_{1}(j+2 n)-S_{1}(3 / 2+j+n)-S_{1}(j) \\
& +S_{1}(3 / 2+j) \\
\mathcal{S}^{(2)}(j, n)=[ & \left.\mathcal{S}^{(1)}(j, n)\right]^{2}-S_{2}(j+2 n)+S_{2}(3 / 2+j+n) \\
& +S_{2}(j)-S_{2}(3 / 2+j)
\end{aligned}
$$

The approximation of $s_{j}^{1}(\omega)$ reads 
TABLE IX. The first four nonvanishing and even eigenvalues of the evolution kernel (1.5) to NNLO accuracy [for color decomposition see Eq. (3.39)]. The values of $v_{j}^{(2)}$ are taken from the nonsinglet result for the deep inelastic structure function $F_{3}[58]$.

\begin{tabular}{ccccc}
\hline \hline$j$ & $C_{F} v_{j}$ & $C_{F}^{2} v_{F, j}+\frac{C_{F}}{2 N_{c}} v_{G, j}$ & $v_{\beta, j}$ & $v_{j}^{(2)}=-\frac{1}{2} \gamma_{j}^{(2)}$ \\
\hline 2 & -2.77778 & 3.41307 & -2.88194 & $-155.614+24.5592 n_{f}+0.220250 n_{f}^{2}$ \\
4 & -4.04444 & 7.15867 & -4.32389 & $-215.118+34.7698 n_{f}+0.295776 n_{f}^{2}$ \\
6 & -4.89048 & 9.82554 & -5.30857 & $-254.562+41.3602 n_{f}+0.342420 n_{f}^{2}$ \\
8 & -5.52910 & 11.86905 & -6.06196 & $-284.650+46.3238 n_{f}+0.375806 n_{f}^{2}$ \\
\hline \hline
\end{tabular}

$$
\begin{aligned}
s_{2}^{(1)}(\omega) \simeq & \frac{13 \omega^{2}}{54}\left(1+0.3642 \omega^{2}+0.1973 \omega^{4}+0.1266 \omega^{6}\right. \\
& \left.+0.0894 \omega^{8}+0.0670 \omega^{10}\right), \\
s_{4}^{(1)}(\omega) \simeq & \frac{83 \omega^{2}}{338}\left(1+0.3694 \omega^{2}+0.2025 \omega^{4}+0.1313 \omega^{6}\right. \\
& \left.+0.0935 \omega^{8}+0.0707 \omega^{10}\right), \\
s_{6}^{(1)}(\omega) \simeq & \frac{143 \omega^{2}}{578}\left(1+0.3716 \omega^{2}+0.2048 \omega^{4}+0.1334 \omega^{6}\right. \\
& \left.+0.0953 \omega^{8}+0.0723 \omega^{10}\right), \\
s_{8}^{(1)}(\omega) \simeq & \frac{73 \omega^{2}}{294}\left(1+0.3727 \omega^{2}+0.2059 \omega^{4}+0.1344 \omega^{6}\right. \\
& \left.+0.0963 \omega^{8}+0.0732 \omega^{10}\right) .
\end{aligned}
$$

$$
\begin{aligned}
s_{j}^{(2)}(\omega= & 1) \equiv\left[s_{j}^{(1)}(\omega=1)\right]^{2}-4 S_{2}(2 j+3)+S_{2}(j+2) \\
& +S_{2}(j+1)+2 \zeta(2) .
\end{aligned}
$$

The quantities in the $\overline{\mathrm{MS}}$ scheme are evaluated from Eqs. (3.16)-(3.23). For $\mu_{f}^{2}=Q^{2}$ the NLO contribution $T_{j}^{(1)}\left(\omega, \mu_{f}^{2} / Q^{2}=1\right)$ reads

$$
\begin{aligned}
T_{0}^{(1)} \simeq & -2\left(1+0.3333 \omega^{2}+0.1873 \omega^{4}+0.1245 \omega^{6}+0.0904 \omega^{8}\right. \\
& \left.+0.0694 \omega^{10}+0.0554 \omega^{12}\right),
\end{aligned}
$$

Note that the prefactor of these series is given by $[1-3 /(5$ $\left.+2 j)^{2}\right] \omega^{2} / 4 \sim \omega^{2} / 4$ and that for $j=\{2,4,6,8\}$ and $|\omega|=0.8$ the relative error of these approximations is about $\{1.2 \%, 2.5 \%, 4.4 \%, 6.7 \%\}$ and increases to $\{6 \%, 10 \%, 14 \%, 19 \%\}$ for $|\omega|=0.9$. The approximation of $s_{j}^{2}(\omega)$ is given by

$$
\begin{aligned}
s_{2}^{(2)}(\omega) \simeq & \frac{\omega^{2}}{243}\left(1+14.7002 \omega^{2}+10.7004 \omega^{4}+7.7684 \omega^{6}\right. \\
& \left.+5.8690 \omega^{8}+4.5967 \omega^{10}\right), \\
s_{4}^{(2)}(\omega) \simeq & \frac{3 \omega^{2}}{2197}\left(1+44.8125 \omega^{2}+33.1180 \omega^{4}+24.3070 \omega^{6}\right. \\
& \left.+18.5325 \omega^{8}+14.6322 \omega^{10}\right), \\
s_{6}^{(2)}(\omega) \simeq & \frac{3 \omega^{2}}{4913}\left(1+100.913 \omega^{2}+75.0187 \omega^{4}+55.3152 \omega^{6}\right. \\
& \left.+42.3458 \omega^{8}+33.5573 \omega^{10}\right), \\
s_{8}^{(2)}(\omega) \simeq & \frac{\omega^{2}}{3087}\left(1+191.007 \omega^{2}+142.411 \omega^{4}+105.261 \omega^{6}\right. \\
& \left.+80.7569 \omega^{8}+64.1255 \omega^{10}\right) .
\end{aligned}
$$

$$
\begin{aligned}
T_{2}^{(1)} \simeq & \frac{\omega^{2}}{9}\left(1+1.2815 \omega^{2}+1.0293 \omega^{4}+0.7770 \omega^{6}+0.5844 \omega^{8}\right. \\
& \left.+0.4436 \omega^{10}+0.3407 \omega^{12}\right)
\end{aligned}
$$$$
T_{4}^{(1)} \simeq \frac{4184 \omega^{4}}{42525}\left(1+1.4076 \omega^{2}+1.4571 \omega^{4}+1.3683 \omega^{6}\right.
$$$$
\left.+1.2370 \omega^{8}+1.1013 \omega^{10}+0.9754 \omega^{12}\right),
$$$$
T_{6}^{(1)} \simeq \frac{96182 \omega^{6}}{2837835}\left(1+1.8560 \omega^{2}+2.3723 \omega^{4}+2.6207 \omega^{6}\right.
$$$$
\left.+2.6949 \omega^{8}+2.6644 \omega^{10}+2.5747 \omega^{12}\right),
$$$$
T_{8}^{(1)} \simeq \frac{568352 \omega^{8}}{57432375}\left(1+2.3324 \omega^{2}+3.5542 \omega^{4}+4.5116 \omega^{6}\right.
$$$$
\left.+5.1921 \omega^{8}+5.6346 \omega^{10}+5.8894 \omega^{12}\right),
$$

while the conformal moments of the factorization logproportional term, i.e., $C_{F} v_{j} T_{j}^{(0)}(\omega)$, are obtained by multiplying the results (D2) with the values of $C_{F} v_{j}$, given in Table IX. At NNLO, only the $\beta_{0}$-proportional term has been evaluated: 


$$
\begin{aligned}
T_{\beta, 0}^{(2) \simeq} & -3\left(1+0.3505 \omega^{2}+0.1785 \omega^{4}+0.1098 \omega^{6}+0.0750 \omega^{8}\right. \\
& \left.+0.0548 \omega^{10}+0.0420 \omega^{12}\right), \\
T_{\beta, 2}^{(2)} \simeq & \frac{4369 \omega^{2}}{8640}\left(1+0.9159 \omega^{2}+0.7298 \omega^{4}+0.5740 \omega^{6}\right. \\
& \left.+0.4566 \omega^{8}+0.3691 \omega^{10}+0.3031 \omega^{12}\right), \\
T_{\beta, 4}^{(2)} \simeq & \frac{2356859 \omega^{4}}{8505000}\left(1+1.3588 \omega^{2}+1.4063 \omega^{4}+1.3333 \omega^{6}\right. \\
& \left.+1.2209 \omega^{8}+1.1022 \omega^{10}+0.9899 \omega^{12}\right), \\
T_{\beta, 6}^{(2)} \simeq & \frac{20352710029 \omega^{6}}{222486264000}\left(1+1.8408 \omega^{2}+2.3592 \omega^{4}\right. \\
& \left.+2.6233 \omega^{6}+2.7190 \omega^{8}+2.7108 \omega^{10}+2.6415 \omega^{12}\right), \\
T_{\beta, 8}^{(2)} \simeq & \frac{363260060687 \omega^{8}}{13676945782500}\left(1+2.3308 \omega^{2}+3.5655 \omega^{4}\right. \\
& \left.+4.5516 \omega^{6}+5.2715 \omega^{8}+5.7582 \omega^{10}+6.0576 \omega^{12}\right) .
\end{aligned}
$$

$$
\begin{aligned}
T_{0}^{\mathrm{CS}(1)} \simeq & -2\left(1+0.2 \omega^{2}+0.0857 \omega^{4}+0.0476 \omega^{6}+0.0303 \omega^{8}\right. \\
& \left.+0.0210 \omega^{10}+0.0154 \omega^{12}\right),
\end{aligned}
$$$$
T_{2}^{\mathrm{CS}(1)} \simeq \frac{\omega^{2}}{9}\left(1+1.4691 \omega^{2}+1.2818 \omega^{4}+1.0443 \omega^{6}\right.
$$$$
\left.+0.8466 \omega^{8}+0.6933 \omega^{10}+0.5752 \omega^{12}\right),
$$$$
T_{4}^{\mathrm{CS}(1)} \simeq \frac{4184 \omega^{4}}{42525}\left(1+1.4102 \omega^{2}+1.4674 \omega^{4}+1.3875 \omega^{6}\right.
$$$$
\left.+1.2644 \omega^{8}+1.1354 \omega^{10}+1.0148 \omega^{12}\right),
$$$$
T_{6}^{\mathrm{CS}(1)} \simeq \frac{96182 \omega^{6}}{2837835}\left(1+1.8373 \omega^{2}+2.3344 \omega^{4}+2.5697 \omega^{6}\right.
$$$$
\left.+2.6372 \omega^{8}+2.6050 \omega^{10}+2.5171 \omega^{12}\right),
$$$$
T_{8}^{\mathrm{CS}(1)} \simeq \frac{568352 \omega^{8}}{57432375}\left(1+2.3052 \omega^{2}+3.4829 \omega^{4}+4.3926 \omega^{6}\right.
$$$$
\left.+5.0299 \omega^{8}+5.4373 \omega^{10}+5.6655 \omega^{12}\right),
$$

To restore the factorization $\log$, one needs

$$
\begin{aligned}
& v_{\beta, 0}^{\Sigma} \simeq-\frac{\omega^{2}}{6}\left(1+0.5657 \omega^{2}+0.3830 \omega^{4}+0.2837 \omega^{6}\right. \\
& \left.+0.2220 \omega^{8}+0.1804 \omega^{10}\right) \\
& v_{\beta, 2}^{\Sigma} \simeq-\frac{83 \omega^{2}}{216}\left(1+0.0488 \omega^{2}+0.0308 \omega^{4}+0.0225 \omega^{6}\right. \\
& \left.+0.0177 \omega^{8}+0.0146 \omega^{10}+0.0123 \omega^{12}\right), \\
& v_{\beta, 4}^{\Sigma} \simeq-\frac{7783 \omega^{4}}{70875}\left(1+0.0261 \omega^{2}+0.0172 \omega^{4}+0.0130 \omega^{6}\right. \\
& \left.+0.0105 \omega^{8}+0.0088 \omega^{10}+0.0076 \omega^{12}\right), \\
& v_{\beta, 6}^{\Sigma} \simeq-\frac{3745727 \omega^{6}}{132432300}\left(1+0.0174 \omega^{2}+0.0118 \omega^{4}\right. \\
& +0.0091 \omega^{6}+0.0075 \omega^{8}+0.0064 \omega^{10} \\
& \left.+0.0056 \omega^{12}\right) \\
& v_{\beta, 8}^{\Sigma} \simeq-\frac{76991788 \omega^{8}}{10854718875}\left(1+0.0130 \omega^{2}+0.0089 \omega^{4}\right. \\
& +0.0070 \omega^{6}+0.0058 \omega^{8}+0.0050 \omega^{10} \\
& \left.+0.0044 \omega^{12}\right) \text {. }
\end{aligned}
$$

while the factorization log-proportional term is the same as in the $\overline{\mathrm{MS}}$ scheme. The NNLO correction $T_{j}^{\mathrm{CS}(2)}\left(\omega, \mu_{f} / Q^{2}\right.$ $=1, \mu_{r} / Q^{2}=1$ ) for $\beta_{0}=0$ reads

$$
\begin{aligned}
T_{0}^{\mathrm{CS}(2)} \simeq & 3.6667\left(1+0.2 \omega^{2}+0.0857 \omega^{4}+0.0476 \omega^{6}\right. \\
& \left.+0.0303 \omega^{8}+0.021 \omega^{10}+0.0154 \omega^{12}\right)_{\mid \beta_{0}=0},
\end{aligned}
$$

$$
\begin{aligned}
T_{2}^{\mathrm{CS}(2)} \simeq & -3.2331 \omega^{2}\left(1+0.6769 \omega^{2}+0.4557 \omega^{4}\right. \\
& +0.3224 \omega^{6}+0.2386 \omega^{8}+0.1832 \omega^{10} \\
& \left.+0.1448 \omega^{12}\right)_{\beta_{0}=0} \\
T_{4}^{\mathrm{CS}(2)} \simeq- & 0.9338 \omega^{4}\left(1+1.0967 \omega^{2}+0.9764 \omega^{4}\right. \\
& +0.8272 \omega^{6}+0.6939 \omega^{8}+0.5838 \omega^{10} \\
& \left.+0.4949 \omega^{12}\right)_{\mid \beta_{0}=0}, \\
T_{6}^{\mathrm{CS}(2)} \simeq & -0.21 \omega^{6}\left(1+1.5133 \omega^{2}+1.6617 \omega^{4}+1.6285 \omega^{6}\right. \\
& \left.+1.5184 \omega^{8}+1.3829 \omega^{10}+1.2459 \omega^{12}\right)_{\mid \beta_{0}=0},
\end{aligned}
$$




$$
\begin{aligned}
T_{8}^{\mathrm{CS}(2)} \simeq & -0.0427 \omega^{8}\left(1+1.905 \omega^{2}+2.4505 \omega^{4}\right. \\
& +2.6858 \omega^{6}+2.7134 \omega^{8}+2.6181 \omega^{10} \\
& \left.+2.4577 \omega^{12}\right)_{\mid \beta_{0}=0} .
\end{aligned}
$$

The $Q^{2}$-independent and $\beta_{0}$-proportional term in the CS scheme is the same as in the $\overline{\mathrm{MS}}$ one, given in Eq. (D12). The factorization and renormalization log-proportional terms appearing in Eq. (3.64) can easily be restored by means of the results from Table IX, Eqs. (D2), (D5), as well as Eqs. (3.68), (D8), (D10), (D11).

The difference between the CS and $\overline{C S}$ schemes arises only from the $\beta_{0}$-proportional terms (3.69). The $\beta_{0}$-proportional NNLO term $T_{\beta, j} \overline{\mathrm{CS}}(2)(\omega)$ reads

$$
\begin{aligned}
T_{\beta, 0}^{\overline{\mathrm{CS}}(2)} \simeq & -3\left(1+0.2 \omega^{2}+0.0857 \omega^{4}+0.0476 \omega^{6}+0.0303 \omega^{8}\right. \\
& \left.+0.0210 \omega^{10}+0.0154 \omega^{12}\right), \\
T_{\beta, 2}^{\overline{\mathrm{CS}}(2)} \simeq & 0.50567 \omega^{2}\left(1+0.8496 \omega^{2}+0.6431 \omega^{4}+0.4900 \omega^{6}\right. \\
& \left.+0.3820 \omega^{8}+0.3048 \omega^{10}+0.2482 \omega^{12}\right), \\
T_{\beta, 4}^{\overline{\mathrm{CS}}(2)} \simeq & 0.277114 \omega^{4}\left(1+1.2512 \omega^{2}+1.2252 \omega^{4}+1.1162 \omega^{6}\right. \\
& \left.+0.9919 \omega^{8}+0.8747 \omega^{10}+0.7710 \omega^{12}\right), \\
T_{\beta, 6}^{\overline{\mathrm{CS}}(2)} \simeq & 0.0914785 \omega^{6}\left(1+1.7236 \omega^{2}+2.1049 \omega^{4}\right. \\
& \left.+2.2550 \omega^{6}+2.2689 \omega^{8}+2.2076 \omega^{10}+2.1078 \omega^{12}\right), \\
T_{\beta, 8}^{\overline{\mathrm{CS}}(2)} \simeq & 0.02656 \omega^{8}\left(1+2.2092 \omega^{2}+3.2413 \omega^{4}+4.0009 \omega^{6}\right. \\
& \left.+4.5064, \omega^{8}+4.8081 \omega^{10}+4.9570 \omega^{12}\right) .
\end{aligned}
$$

where the coefficients $\alpha, \ldots, \delta$ are determined from a fit of the lowest moments. In this way, we obtain an approximation that is better than $1 \%$ for all moments. Now we can recover an approximate expression for the hard-scattering amplitude as a convolution by the following recipe.

Substitute the LO Wilson coefficients by the corresponding hard-scattering amplitude:

$$
\frac{2 j+3}{(j+1)(j+2)} \rightarrow \frac{1}{2(1-x)}
$$

Restore the kernels:

$$
\begin{gathered}
\text { cons } \rightarrow \text { cons } \mathbf{I}, \quad \frac{1}{(j+1)(j+2)} \rightarrow v_{a}(x, y), \\
S_{1}(1+j) \rightarrow-\frac{1}{2}\left[v_{b}(x, y)\right]_{+}+1 \mathbf{I} .
\end{gathered}
$$

Consider the multiplication of the conformal moments given above, which corresponds to convolution in the momentum fraction space.

Here we have introduced a shorthand notation for the identity $\mathbf{I} \equiv \delta(x-y)$. In the $\overline{\mathrm{MS}}$ scheme, using Eq. (3.32) and the recipe given above we restore the exact expression (3.28) in the momentum fraction space. For the CS scheme, using the improved form (4.1), we get a good approximation of Eq. (3.45), for the lowest moments also, by taking

$$
\begin{gathered}
\alpha=\frac{83}{10}+8 \zeta(2)-26 \ln (2) \approx 3.438, \\
\gamma=-\frac{73}{5}-12 \zeta(2)+48 \ln (2) \approx-1.068, \beta=\delta=0,
\end{gathered}
$$

and the hard-scattering part reads 


$$
\begin{aligned}
T_{F}^{\mathrm{CS}(1)}(x) \approx & T^{(0)} \otimes\left\{\frac{1}{2}\left[v_{b}\right]_{+} \otimes\left(\frac{1}{2}\left[v_{b}\right]_{+}-6.273 \mathbf{I}\right)-2.952 \mathbf{I}+3.438 v_{a}-1.068 v_{a} \otimes v_{a}\right\}(x) \\
\approx & \frac{0.25 \ln ^{2}(1-x)-2.136 \ln (1-x)-8.224}{2(1-x)}+\frac{\ln (1-x)[0.25 \ln (1-x)-6.642]}{2 x} \\
& +1.068 \frac{\mathrm{Li}_{2}(x)-\mathrm{Li}_{2}(1)}{2(1-x)} .
\end{aligned}
$$

After analytical continuation in $x$ this result corresponds to the NLO correction of the deeply virtual Compton scattering for the quark-quark channel in the parity odd sector.

[1] G.P. Lepage and S.J. Brodsky, Phys. Rev. D 22, 2157 (1980).

[2] S.J. Brodsky and G.P. Lepage, Phys. Rev. D 24, 1808 (1981).

[3] A.V. Efremov and A.V. Radyushkin, Phys. Lett. 94B, 245 (1980).

[4] D. Müller, D. Robaschik, B. Geyer, F.-M. Dittes, and J. Hořejši, Fortschr. Phys. 42, 101 (1994).

[5] F. Del Aguila and M.K. Chase, Nucl. Phys. B193, 517 (1981).

[6] E. Braaten, Phys. Rev. D 28, 524 (1983).

[7] E.P. Kadantseva, S.V. Mikhailov, and A.V. Radyushkin, Sov. J. Nucl. Phys. 44, 326 (1986).

[8] P. Gosdzinsky and N. Kivel, Nucl. Phys. B521, 274 (1998).

[9] A.V. Belitsky and A. Schäfer, Nucl. Phys. B527, 235 (1998).

[10] B. Melić, B. Nižić, and K. Passek, Phys. Rev. D 65, 053020 (2002).

[11] M.H. Sarmadi, Phys. Lett. 143B, 471 (1984).

[12] F.-M. Dittes and A.V. Radyushkin, Phys. Lett. 134B, 359 (1984).

[13] S.V. Mikhailov and A.V. Radyushkin, Nucl. Phys. B254, 89 (1985).

[14] S.V. Mikhailov and A.V. Radyushkin, Nucl. Phys. B273, 297 (1986).

[15] D. Müller, Phys. Rev. D 49, 2525 (1994).

[16] D. Müller, Phys. Rev. D 51, 3855 (1995).

[17] CELLO Collaboration, H.-J. Behrend et al., Z. Phys. C 49, 401 (1991).

[18] CLEO Collaboration, J. Gronberg et al., Phys. Rev. D 57, 33 (1998).

[19] P. Kroll and M. Raulfs, Phys. Lett. B 387, 848 (1996).

[20] A.V. Radyushkin and R. Ruskov, Phys. Lett. B 374, 173 (1996).

[21] F.-G. Cao, T. Huang, and B.-Q. Ma, Phys. Rev. D 53, 6852 (1996).

[22] A.V. Radyushkin and R. Ruskov, Nucl. Phys. B481, 625 (1996).

[23] I.V. Musatov and A.V. Radyushkin, Phys. Rev. D 56, 2713 (1997).

[24] N.G. Stefanis, W. Schroers, and H.C. Kim, Phys. Lett. B 449, 299 (1999).

[25] N.G. Stefanis, W. Schroers, and H.C. Kim, Eur. Phys. J. C 18, 137 (2000).

[26] A.P. Bakulev, S.V. Mikhailov, and N.G. Stefanis, Phys. Lett. B 508, 279 (2001).

[27] V.M. Braun and I.E. Halperin, Phys. Lett. B 328, 457 (1994).

[28] R. Jakob, P. Kroll, and M. Raulfs, J. Phys. G 22, 45 (1996).
[29] S. Ong, Phys. Rev. D 52, 3111 (1995).

[30] M. Diehl, P. Kroll, and C. Vogt, Eur. Phys. J. C 22, 439 (2001).

[31] R.D. Field, R. Gupta, S. Otto, and L. Chang, Nucl. Phys. B186, 429 (1981).

[32] F.-M. Dittes and A.V. Radyushkin, Sov. J. Nucl. Phys. 34, 293 (1981).

[33] F.-M. Dittes, Ph.D. thesis, Moscow University, 1982.

[34] M.H. Sarmadi, Ph.D. thesis, Pittsburgh University, 1982.

[35] A.V. Radyushkin and R.S. Khalmuradov, Sov. J. Nucl. Phys. 42, 289 (1985).

[36] E. Braaten and S.-M. Tse, Phys. Rev. D 35, 2255 (1987).

[37] B. Melić, B. Nižić, and K. Passek, Phys. Rev. D 60, 074004 (1999).

[38] B. Nižić, Phys. Rev. D 35, 80 (1987).

[39] D. Müller, Phys. Rev. D 58, 054005 (1998).

[40] D. Müller, Phys. Rev. D 59, 116003 (1999).

[41] S. Ferrara, R. Gatto, and A.F. Grillo, Nucl. Phys. B34, 349 (1971).

[42] S. Ferrara, R. Gatto, and A.F. Grillo, Phys. Rev. D 5, 3102 (1972).

[43] E.B. Zijlstra and W.L. van Neerven, Nucl. Phys. B417, 61 (1994); B426, 245(E) (1994).

[44] A.V. Belitsky and D. Müller, Phys. Lett. B 417, 129 (1997).

[45] A.V. Belitsky and D. Müller, Nucl. Phys. B527, 207 (1998).

[46] A.V. Belitsky and D. Müller, Nucl. Phys. B537, 397 (1999).

[47] A.V. Belitsky, D. Müller, and A. Schäfer, Phys. Lett. B 450, 126 (1999).

[48] A.V. Belitsky and D. Müller, Phys. Rev. D 65, 054037 (2002).

[49] R.J. Crewther, Phys. Rev. Lett. 28, 1421 (1972); Phys. Lett. B 397, 137 (1997); S.L. Adler, C.G. Callan, D.J. Gross, and R. Jackiw, Phys. Rev. D 6, 2982 (1972); D.J. Broadhurst and A.L. Kataev, Phys. Lett. B 315, 179 (1993).

[50] S.J. Brodsky, Y. Frishman, G.P. Lepage, and C. Sachradja, Phys. Lett. 91B, 239 (1980).

[51] Yu.M. Makeenko, Sov. J. Nucl. Phys. 33, 440 (1981).

[52] T. Ohrndorf, Nucl. Phys. B198, 26 (1982).

[53] I.I. Balitsky and V.M. Braun, Nucl. Phys. B311, 541 (1989).

[54] E.G. Floratos, D.A. Ross, and C.T. Sachrajda, Nucl. Phys. B129, 66 (1977); B139, 545(E) (1978).

[55] A. Gonzales-Arroyo, C. Lopez, and F.J. Yndurain, Nucl. Phys. B153, 161 (1979).

[56] G. Curci, W. Furmanski, and R. Petronzio, Nucl. Phys. B175, 27 (1980). 
[57] E.G. Floratos, R. Lacaze, and C. Kounnas, Phys. Lett. 98B, 89 (1981).

[58] A. Retey and J.A. Vermaseren, Nucl. Phys. B604, 281 (2001).

[59] A.V. Belitsky and D. Müller, Nucl. Phys. B527, 207 (1998).

[60] L. Mankiewicz, G. Piller, E. Stein, M. Vänttinen, and T. Weigl, Phys. Lett. B 425, 186 (1998).

[61] X. Ji and J. Osborne, Phys. Rev. D 57, 1337 (1998).

[62] X. Ji and J. Osborne, Phys. Rev. D 58, 094018 (1998).

[63] O.V. Tarasov, A.A. Vladimirov, and A.Yu. Zharkov, Phys. Lett. 93B, 429 (1980); S.A. Larin and J.A.M. Vermaseren, Phys. Lett. B 303, 334 (1993).

[64] UKQCD Collaboration, L. Del Debbio, M. Di Pierro, A. Dougall, and C. Sachrajda, Nucl. Phys. B (Proc. Suppl.) 83, 235 (2000).

[65] V.L. Chernyak and I.R. Zhitnitsky, Phys. Rep. 112, 173 (1984).

[66] V.M. Braun and I.E. Filyanov, Z. Phys. C 44, 157 (1989).

[67] S.J. Brodsky, G.P. Lepage, and P.B. Mackenzie, Phys. Rev. D 28, 228 (1983).

[68] S.J. Brodsky, C.R. Ji, A. Pang, and D.G. Robertson, Phys. Rev.
D 57, 245 (1998).

[69] M. Meyer-Hermann, M. Maul, L. Mankiewicz, E. Stein, and A. Schafer, Phys. Lett. B 383, 463 (1996).

[70] V.M. Braun and I.E. Filyanov, Z. Phys. C 48, 239 (1990); P. Ball, J. High Energy Phys. 01, 010 (1999).

[71] A. Khodjamirian, Eur. Phys. J. C 6, 477 (1999).

[72] S.G. Gorishnii and S.A. Larin, Phys. Lett. B 172, 109 (1986); S.A. Larin and J.A.M. Vermaseren, ibid. 259, 345 (1991).

[73] D.J. Broadhurst and A.L. Kataev, Phys. Lett. B 315, 179 (1993); 544, 154 (2002); A.L. Kataev and V.V. Starshenko, Mod. Phys. Lett. A 10, 235 (1995).

[74] N. Nakanishi, Graph Theory and Feynman Integrals (Gordon and Breach, New York, 1971).

[75] A. Devoto and D.W. Duke, Riv. Nuovo Cimento 7, 1 (1984).

[76] J. Blümlein and S. Kurth, Phys. Rev. D 60, 014018 (1999).

[77] J.A.M. Vermaseren, Int. J. Mod. Phys. A 14, 2037 (1999).

[78] I.M. Gel'fand and G.E. Shilov, Generalized Functions (Academic Press, New York, 1964), Vol. I. 